\title{
A Photochemical Dehydrogenative Strategy for Direct and Regioselective Dimerization of BODIPY Dyes
}

Dandan Wang, ${ }^{\dagger}$ Qinghua Wu,,$^{\dagger}$ Xiankang Zhang,,$^{\dagger}$ Wenqing Wang, ${ }^{\dagger}$ Erhong Hao,,${ }^{,}$

$$
\text { and Lijuan Jiao }{ }^{*},
$$

${ }^{\dagger}$ Laboratory of Functional Molecular Solids, Ministry of Education; School of Chemistry and Materials Science, Anhui Normal University, Wuhu, 241002, China

*To whom correspondence should be addressed.

E-mail: haoehong@ahnu.edu.cn, jiao421@ahnu.edu.cn

\section{Contents:}

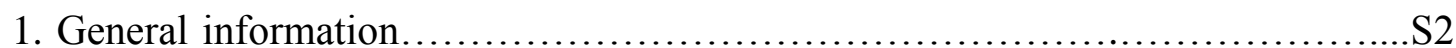

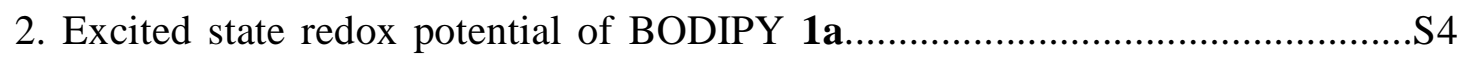

3. Calculated $\Delta \mathrm{G}$ value of the photoinduced transferprocess........................S5

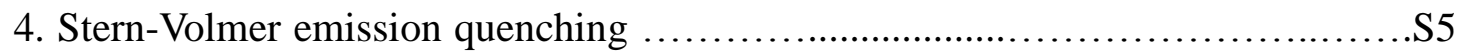

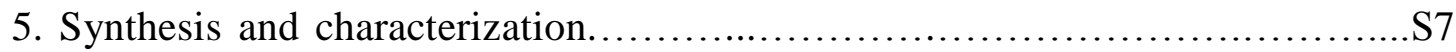

6. Crystal data........................................................... 18

7. Photophysical properties................................................. 23

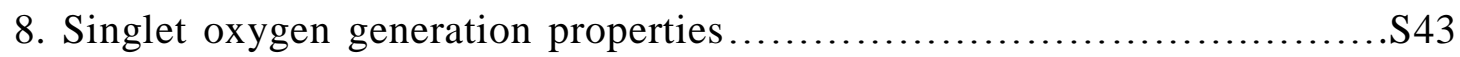

9. Copies of ${ }^{1} \mathrm{H}$ and ${ }^{13} \mathrm{C}$ NMR spectra..................................... S46 


\section{General information}

Reagents and solvents were used as received from commercial suppliers unless noted otherwise. All reactions were performed in oven-dried or flame-dried glassware unless otherwise stated, and were monitored by TLC using $0.25 \mathrm{~mm}$ silica gel plates with UV indicator (60F-254). The light source is WP-TEC-1020SL optical parallel reactor. ${ }^{1} \mathrm{H}$ and ${ }^{13} \mathrm{C}$ NMR were recorded on a $300 \mathrm{MHz}, 400 \mathrm{MHz}$ or $500 \mathrm{MHz}$ NMR spectrometer at room temperature. Chemical shifts $(\delta)$ are given in ppm relative to $\mathrm{CDCl}_{3}(7.26 \mathrm{ppm}$ for ${ }^{1} \mathrm{H}$ and $77 \mathrm{ppm}$ for ${ }^{13} \mathrm{C}$ ). The EPR spectra were obtained using a Bruker EMX plus6/1 variable-temperature X-band apparatus. High-resolution mass spectra (HRMS) were obtained using APCI-TOF, ESI-TOF or MAIDI-TOF in positive mode.

UV-visible absorption spectra and Fluorescence emission spectra were recorded on a commercial spectrophotometer (Shimadzu UV-2450 and Edinburgh FS5 spectrometers, 190-900 nm scan range). Relative fluorescence quantum efficiencies of these BODIPYs were obtained by comparing the areas under the corrected emission spectrum of the test sample in various solvents with Cresyl violet perchlorate $(\Phi=0.54$ in methanol $)^{1}$, Rhodamine B $(\Phi=0.49 \text { in ethanol })^{2}$, Fluorescein $(\Phi=0.9$ in $0.1 \mathrm{M}$ $\mathrm{NaOH})^{3}$, 1,7-diphenyl-3,5-di(4-methoxyphenyl)-azadipyrromethene $(\Phi=0.36$ in chloroform $)^{4}$ and Indocyanine Green $(\Phi=0.12 \text { in dimethyl sulfoxide })^{5}$. Non-degassed, spectroscopic grade solvents and a $10 \mathrm{~mm}$ quartz cuvette were used. Dilute solutions $(0.01<\mathrm{A}<0.05)$ were used to minimize the reabsorption effects. Quantum yields were determined using the following equation:

$$
\Phi_{x}=\Phi_{r} \times \frac{F_{x}}{F_{r}} \times \frac{1-10^{-A_{r}\left(\lambda_{\mathrm{ex}}\right)}}{1-10^{-A_{x}\left(\lambda_{\mathrm{ex}}\right)}} \times \frac{n_{x}^{2}}{n_{r}^{2}}
$$

Where the subscripts $x$ and $r$ refer respectively to sample $x$ and reference (standard) fluorophore $r$ with known quantum yield $\Phi_{r}$ in a specific solvent; $F$ stands for the spectrally corrected, integrated fluorescence spectra; $A\left(\lambda_{\mathrm{ex}}\right)$ denotes the absorbance at the used excitation wavelength $\lambda_{\mathrm{ex}} ; n$ represents the refractive index of the solvent (in principle at the average emission wavelength).

Crystals of 2a, 3a, 2b, 3d, 8 and 9a suitable for X-ray analysis were obtained by slow evaporation of their dichloromethane solutions. The vial containing this solution was 
placed, loosely capped, to promote the crystallization. A suitable crystal was chosen and mounted on a glass fiber using grease. The suitable crystals were selected and mounted on a suitable support. Data were collected using an Bruker APEX-II ${ }^{6}$ CCD diffractometer operating at $T=293(2) \mathrm{K}$. The determination of unit cell parameters and data collections were performed with Mo K $\alpha$ radiation $(\lambda)$ at $0.71073 \AA$. The total number of runs and images was based on the strategy calculation from the program APEX2 (Bruker). ${ }^{7}$ The structure was solved by the structure solution program Olex2 (Dolomanov et al., 2009) ${ }^{8}$ and the model was refined with version 2014/7 of ShelXL (Sheldrick, 2015) ${ }^{9}$ using full matrix least squares on $\mathbf{F}^{2}$ minimisation. CCDC-1568525 (2a), CCDC-1568460 (3a), CCDC-1568472 (2b), CCDC-1568471 (3d), CCDC1916022 (8) and CCDC-2018807 (9a) contain the supplementary crystallographic data for this paper. These data can be obtained free of charge from The Cambridge Crystallographic Data Centre via www.ccdc.cam.ac.uk/data_request/cif.

CW X-band EPR spectra for radicals were acquired on Bruker EMX instrument. EPR spectra of various mixtures were recorded after irradiation of $10 \mathrm{~W}$ white LED for $1 \mathrm{~h}$ at room temperature. 


\section{Excited state redox potential of BODIPY 1a}

Excited state reduction potential of BODIPY 1a was estimated to be - $0.71 \mathrm{~V}$ according to the following equations: ${ }^{10}$

$\mathrm{E}_{1 / 2}\left(\mathbf{1 a}^{+\cdot} / \mathbf{1} \mathbf{a}^{*}\right)=\mathrm{E}^{\mathrm{ox}}-\mathrm{E}_{0,0}$

Where $\mathrm{E}^{\mathrm{ox}}$ was obtained from its cyclic voltammetry spectrum (Figure $\mathrm{S} 1$ ); $\mathrm{E}_{0,0}$ was caculated from its photoluminescence maximum (512 nm as shown in Figure S2) using the equation $\mathrm{E}_{0,0}=\mathrm{hc} / \lambda_{\max }=1240 / \lambda_{\max }$.

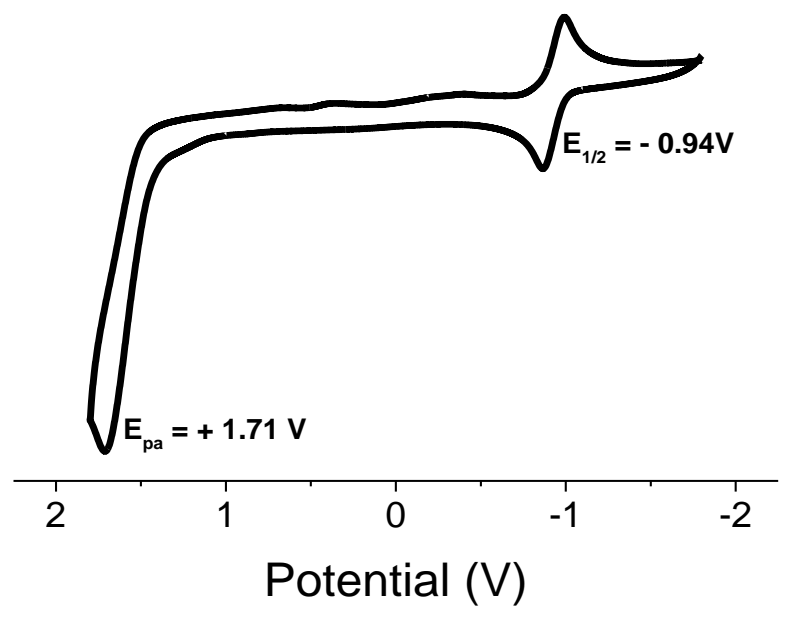

Figure S1. Cyclic voltammetry of BODIPY 1a (2 mM in dichloromethane). $E_{o x}=+$ $1.71 \mathrm{~V}$ vs. SCE)

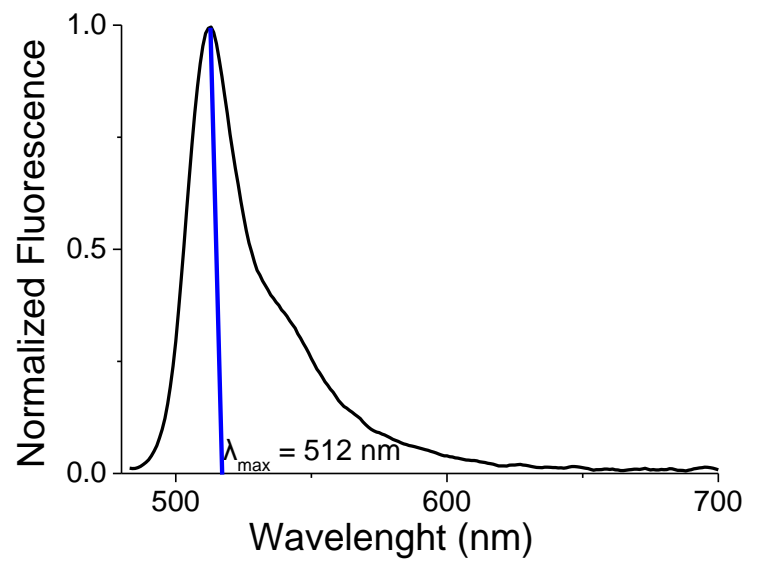

Figure S2. Fluorescence of BODIPY 1a in dichloromethane 


\section{Calculated $\Delta G$ value of the photoinduced transfer process}

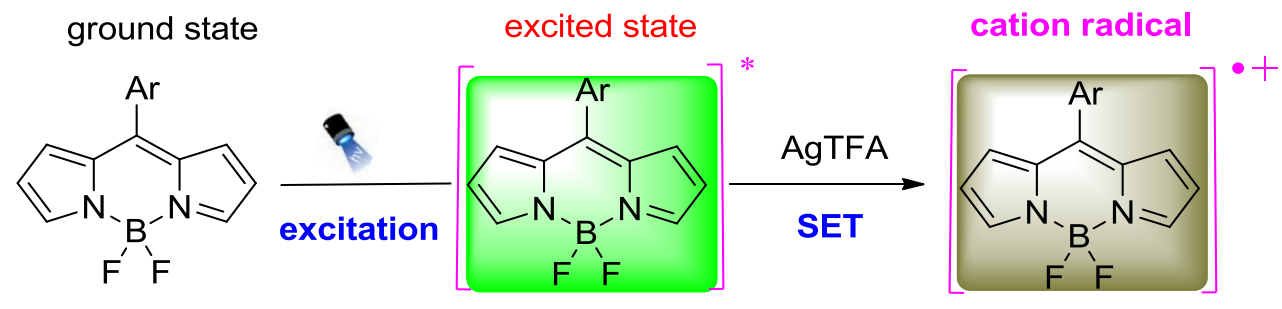

$1 \mathbf{a}$

The change in the free energy $(\Delta \mathrm{G})$ of the electron transfer from the excited state BODIPY 1a to oxidant AgTFA was calculated to be $-45.1 \mathrm{kcal} / \mathrm{mol}$ based on RehmWeller equation:

$\Delta \mathrm{G}=f_{\mathrm{c}}\left[\mathrm{E}_{\text {ox }}(\mathbf{1 a})-\mathrm{E}_{\mathrm{red}}(\mathrm{AgTFA})\right]-\mathrm{E}_{0,0}(\mathbf{1 a})$

Where $f_{\mathrm{c}}$ is the Faraday constant, the oxidation potential $\mathrm{E}_{\mathrm{ox}}$ of $1 \mathbf{a}$ is $+1.71 \mathrm{~V}$, the reduction potential $\mathrm{E}_{\text {red }}$ of the oxidant AgTFA is $0.80 \mathrm{~V}$ and active excitation energy $\mathrm{E}_{0,0}$ was caculated from its photoluminescence maximum $(512 \mathrm{~nm}$ as shown in Figure $\mathrm{S} 2)$

\section{Stern-Volmer emission quenching}

Steady-state emission spectra were recorded using Edinburgh FS5 spectrometers. To a degassed acetonitrile solution of BODIPY $1 \mathbf{a}\left(1 \times 10^{-5} \mathrm{M}\right)$ was added various amount of AgTFA $(0-0.04 \mathrm{M})$. The emission spectra of $\mathbf{1 a}$ was then collected under excitation at $480 \mathrm{~nm}$ as shown in Figure S3a.

The emission intensity of $\mathbf{1 a}$ at $524 \mathrm{~nm}$ was used to determine the Stern-Volmer quenching values. The Stern-Volmer plot, as shown in Figure S3b, shows a linear correlation between the amounts of AgTFA and the ratio $\mathrm{I}_{0} / \mathrm{I} .{ }^{11-12}$ 

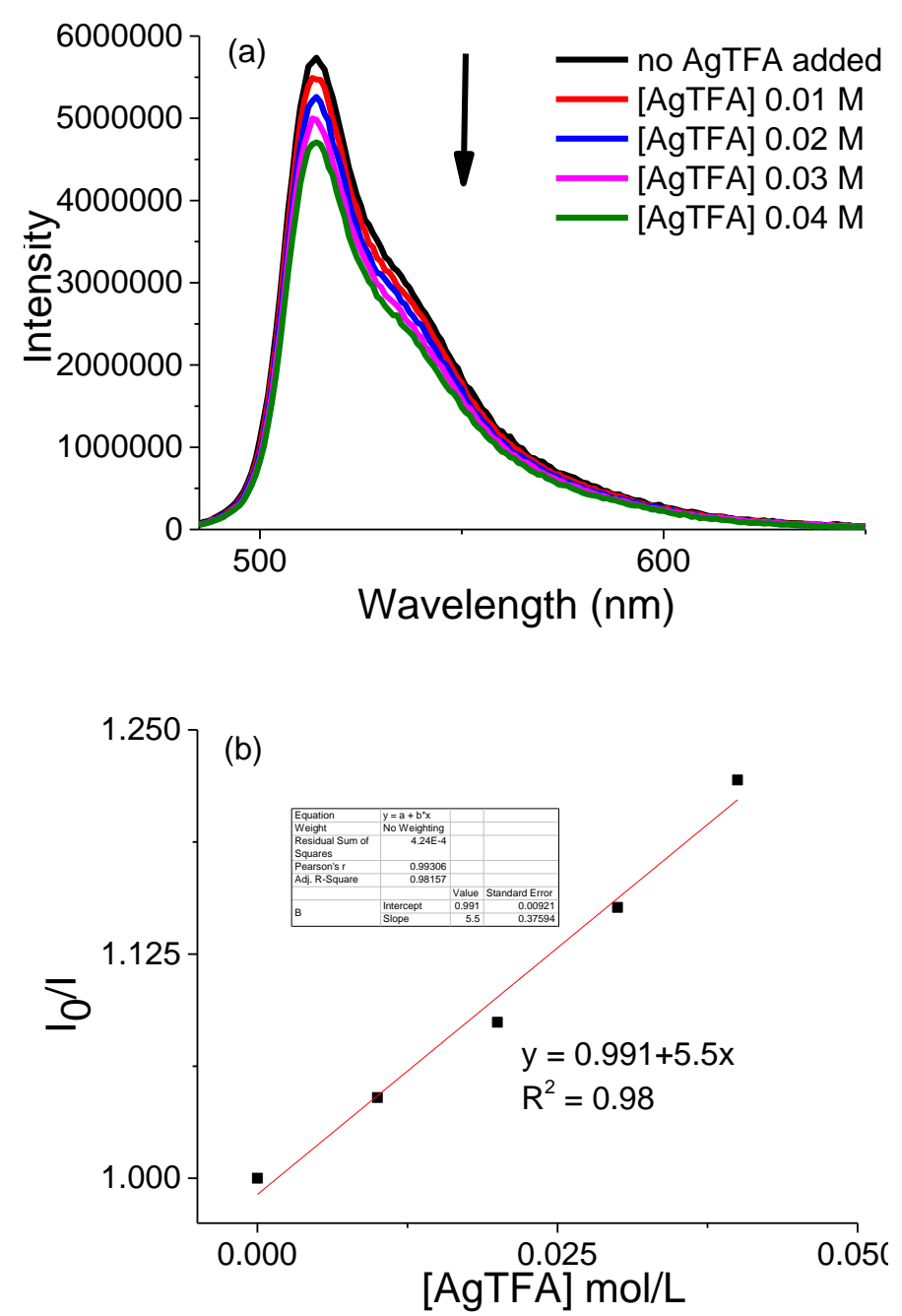

Figure S3. (a) The emission spectra of BODIPY $1 \mathbf{a}\left(1 \times 10^{-5} \mathrm{M}\right)$ in acetonitrile at 25 ${ }^{\circ} \mathrm{C}$ with increasing amounts of $\operatorname{AgTFA}(0-0.04 \mathrm{M})$ under excitation at $480 \mathrm{~nm}$. (b) The Stern-Volmer plot of 1a vs. [AgTFA]. 


\section{Synthesis and characterization}

Table S1. Optimization of the Reaction Conditions for Light-Induced Dimerization. ${ }^{[a]}$

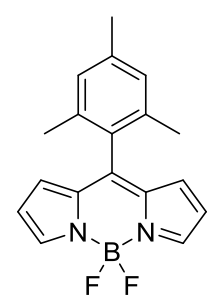

$1 \mathrm{a}$

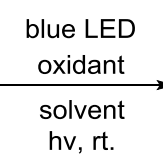

hv, rt.

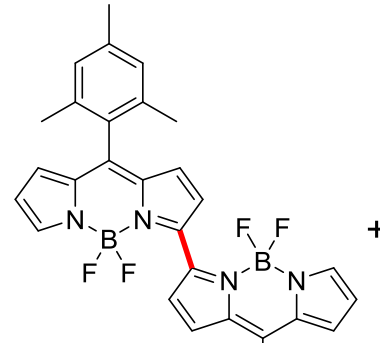

$2 \mathbf{a}$

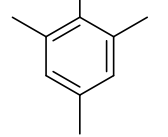

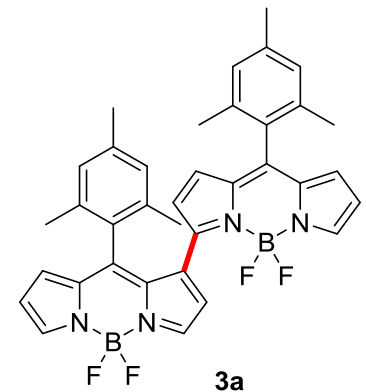

$3 a$

\begin{tabular}{|c|c|c|c|c|}
\hline entry & solvent & oxidant & $2 \mathbf{a}(\%)^{[b]}$ & $3 \mathbf{a}(\%)$ \\
\hline $1[\mathrm{c}]$ & acetonitrile & $\mathrm{FeCl}_{3}$ & -- & -- \\
\hline 2 & acetonitrile & $\mathrm{Cu}(\mathrm{OAc})_{2}$ & 16 & 4 \\
\hline 3 & acetonitrile & $\mathrm{O}_{2}$ & trace & trace \\
\hline $4[d]$ & acetonitrile & $\mathrm{Ar}$ & NR & NR \\
\hline 5 & acetonitrile & air & trace & trace \\
\hline 6 & acetonitrile & $\mathrm{CBrCl}_{3}$ & trace & trace \\
\hline $7^{[\mathrm{e}]}$ & acetonitrile & $\left(\mathrm{NH}_{4}\right)_{2} \mathrm{~S}_{2} \mathrm{O}_{8}$ & 10 & 5 \\
\hline 8 & acetonitrile & $\mathrm{AgNO}_{3}$ & 15 & 6 \\
\hline 9 & acetonitrile & $\mathrm{AgOAc}$ & 63 & 14 \\
\hline 10 & acetonitrile & AgTFA & 71 & 18 \\
\hline $11^{[\mathrm{f}]}$ & acetonitrile & AgTFA & NR & NR \\
\hline 12 & acetone & AgTFA & 9 & 3 \\
\hline 13 & DMSO & AgTFA & $\mathrm{NR}$ & NR \\
\hline 14 & THF & AgTFA & $\mathrm{NR}$ & NR \\
\hline 15 & toluene & AgTFA & NR & NR \\
\hline
\end{tabular}

[a] Reaction conditions: $1 \mathrm{a}(0.1 \mathrm{mmol}), 1.0 \mathrm{~mL}$ of solvent, oxidant (4.0 equiv), $10 \mathrm{~W}$ blue LED irradiation for 48 h. [b] Isolated yields based on 1a. [c] Decomposition of 1a. [d] NR = No reaction occurred, 1 a was recovered. [e] Reaction time 10 h. [f] In dark. 
BODIPYs 1a-e were synthesized by following the procedures described in our previous pa per. $^{13}$

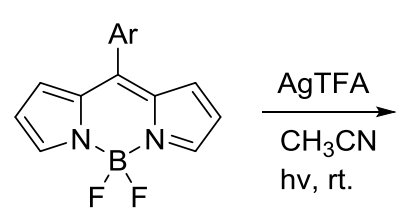

1

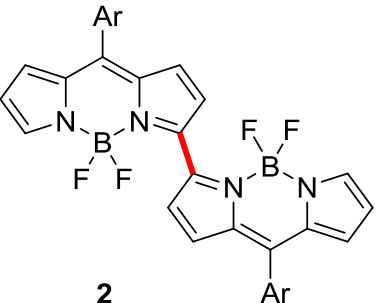

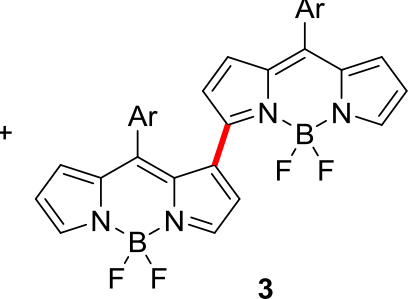

General method for light-induced dimerization of BODIPYs 1: To a solution of 1 $(0.1 \mathrm{mmol})$ in distilled acetonitrile $(1 \mathrm{~mL})$ was added AgTFA ( $88 \mathrm{mg}, 4$ equiv, 0.4 $\mathrm{mmol}$ ). The reaction mixture was stirred at room tempreture for $48 \mathrm{~h}$ under the irridiation of a $10 \mathrm{~W}$ blue light LED $(450 \mathrm{~nm})$. The reaction mixture was then evaporated under vacuum and the residue was purified by column chromatography on silica gel using petroleum ether/dichloromethane as eluent to give dimers $\mathbf{2}$ and $\mathbf{3}$.
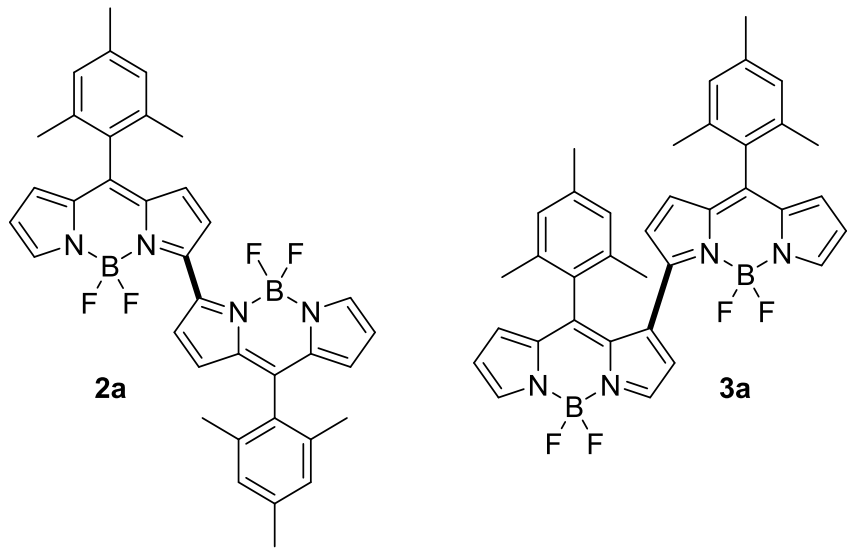

BODIPY dimers 2a and 3a were synthesized from BODIPY 1a $(31 \mathrm{mg}, 0.1 \mathrm{mmol})$ using above general method:

BODIPY dimer 2a as a dark blue solid in $71 \%$ yield (22 $\mathrm{mg}$ ) after silica column chromatography (silica, petroleum ether/dichloromethane: 2:1, v/v). ${ }^{1} \mathrm{H}$ NMR (500 $\left.\mathrm{MHz}, \mathrm{CDCl}_{3}\right) \delta: 7.89(\mathrm{~s}, 2 \mathrm{H}), 7.53(\mathrm{~d}, J=4.4 \mathrm{~Hz}, 2 \mathrm{H}), 6.98(\mathrm{~s}, 4 \mathrm{H}), 6.73(\mathrm{~d}, J=4.4 \mathrm{~Hz}$, $2 \mathrm{H}), 6.70(\mathrm{~d}, J=4.0 \mathrm{~Hz}, 2 \mathrm{H}), 6.49(\mathrm{~d}, J=3.9 \mathrm{~Hz}, 2 \mathrm{H}), 2.38(\mathrm{~s}, 6 \mathrm{H}), 2.16(\mathrm{~s}, 12 \mathrm{H}) .{ }^{13} \mathrm{C}$ NMR (126 MHz, $\left.\mathrm{CDCl}_{3}\right) \delta: 148.2,146.4,144.7,139.0,138.2,136.7,135.9,130.0$, 129.8, 128.3, 124.1, 119.3, 21.3, 20.2. HRMS calcd for $\mathrm{C}_{36} \mathrm{H}_{32} \mathrm{~B}_{2} \mathrm{~F}_{3} \mathrm{~N}_{4}[\mathrm{M}-\mathrm{F}]^{+}$: 599.2765, found: 599.2751 . 
BODIPY dimer 3a as a dark purple solid in $18 \%$ yield $(5 \mathrm{mg})$ after silica column chromatography (silica, petroleum ether/dichloromethane: 1:1, v/v). ${ }^{1} \mathrm{H}$ NMR (500 $\left.\mathrm{MHz}, \mathrm{CDCl}_{3}\right) \delta: 8.00(\mathrm{~s}, 1 \mathrm{H}), 7.95(\mathrm{~s}, 1 \mathrm{H}), 7.79(\mathrm{~s}, 1 \mathrm{H}), 7.14(\mathrm{~s}, 1 \mathrm{H}), 6.94(\mathrm{~s}, 2 \mathrm{H}), 6.64$ (s, 2H), 6.60-6.58 (m, 2H), 6.46-6.44 (m, 2H), 6.07 (d, J=4.2 Hz, 1H), 5.55 (d, J = 4.2 $\mathrm{Hz}, 1 \mathrm{H}), 2.36$ (s, 3H), 2.18 (s, 3H), 2.09 (s, 6H), 2.07 (s, 6H). ${ }^{13} \mathrm{C}$ NMR (125 MHz, $\left.\mathrm{CDCl}_{3}\right) \delta: 151.0,147.6,145.5,145.0,143.3,143.0,139.0,138.9,137.2,136.6,136.4$, 136.0, 134.9, 132.6, 131.4, 130.4, 129.9, 128.8, 128.2, 128.1, 127.9, 121.9, 119.0, 118.5, 21.2, 21.2, 20.1, 19.9. HRMS calcd for $\mathrm{C}_{36} \mathrm{H}_{32} \mathrm{~B}_{2} \mathrm{~F}_{3} \mathrm{~N}_{4}[\mathrm{M}-\mathrm{F}]^{+}$: 599.2765, found: 599.2756
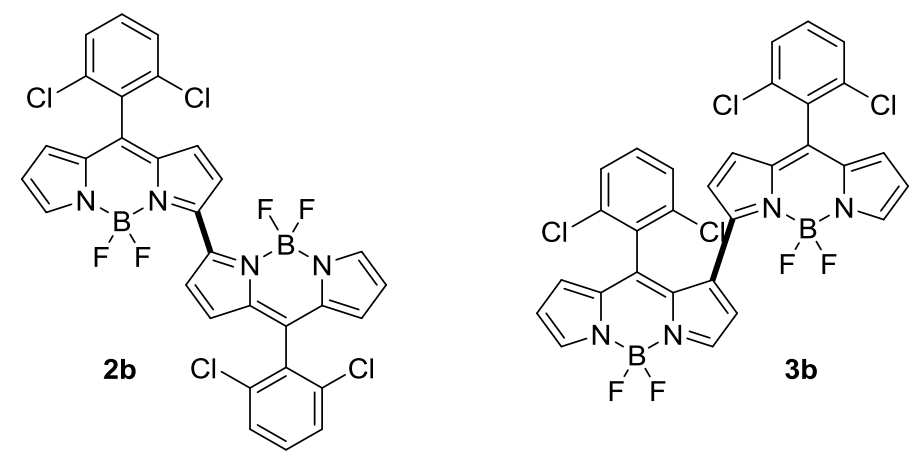

BODIPY dimers $\mathbf{2 b}$ and $\mathbf{3 b}$ were synthesized from BODIPY $\mathbf{1 b}(34 \mathrm{mg}, 0.1 \mathrm{mmol})$ using above general method:

BODIPY dimer $\mathbf{2 b}$ as a dark red solid in $46 \%$ yield $(15 \mathrm{mg}$ ) after silica column chromatography (silica, petroleum ether/dichloromethane: 2:1, v/v). ${ }^{1} \mathrm{H}$ NMR (500 $\left.\mathrm{MHz} \mathrm{CDCl}_{3}\right) \delta: 7.94(\mathrm{~s}, 2 \mathrm{H}), 7.62(\mathrm{~d}, J=4.4 \mathrm{~Hz}, 2 \mathrm{H}), 7.51(\mathrm{~d}, J=7.6 \mathrm{~Hz}, 4 \mathrm{H}), 7.46-$ $7.43(\mathrm{~m}, 2 \mathrm{H}), 6.74-6.73(\mathrm{~m}, 4 \mathrm{H}), 6.55(\mathrm{~d}, J=4.1 \mathrm{~Hz}, 2 \mathrm{H}) .{ }^{13} \mathrm{C} \mathrm{NMR}\left(126 \mathrm{MHz}, \mathrm{CDCl}_{3}\right)$ $\delta: 148.6,146.2,139.3,137.7,135.6,131.5,131.4,129.9,129.5,128.4,124.8,120.1$. HRMS calcd for $\mathrm{C}_{30} \mathrm{H}_{16} \mathrm{~B}_{2} \mathrm{Cl}_{4} \mathrm{~F}_{3} \mathrm{~N}_{4}[\mathrm{M}-\mathrm{F}]^{+}:$651.0267, found: 651.0266 .

BODIPY dimer $\mathbf{3 b}$ as a bottle green solid in $22 \%$ yield $(7 \mathrm{mg})$ after silica column chromatography (silica, petroleum ether/dichloromethane: 1:1, v/v). ${ }^{1} \mathrm{H}$ NMR (500 $\left.\mathrm{MHz} \mathrm{CDCl}_{3}\right) \delta: 8.03(\mathrm{~s}, 1 \mathrm{H}), 8.00(\mathrm{~s}, 1 \mathrm{H}), 7.89$ (s, 1H), 7.48-7.47 (m, 2H), 7.44-7.41 $(\mathrm{m}, 1 \mathrm{H}), 7.17(\mathrm{~s}, 4 \mathrm{H}), 6.63(\mathrm{~d}, J=4.0 \mathrm{~Hz}, 2 \mathrm{H}), 6.54-6.49(\mathrm{~m}, 2 \mathrm{H}), 6.11(\mathrm{~d}, J=4.2 \mathrm{~Hz}$, $1 \mathrm{H}), 5.98(\mathrm{~d}, J=4.1 \mathrm{~Hz}, 1 \mathrm{H}) .{ }^{13} \mathrm{C} \mathrm{NMR}\left(125 \mathrm{MHz}, \mathrm{CDCl}_{3}\right) \delta: 150.9,146.3,145.6$, 144.1, 140.5, 139.0, 136.0, 135.2, 134.6, 132.3, 131.9, 131.5, 131.3, 131.2, 129.3, 128.3, 127.9, 122.0, 121.8, 119.5. HRMS calcd for $\mathrm{C}_{30} \mathrm{H}_{16} \mathrm{~B}_{2} \mathrm{Cl}_{4} \mathrm{~F}_{3} \mathrm{~N}_{4}[\mathrm{M}-\mathrm{F}]^{+}$: 651.0267 , found: 651.0287 

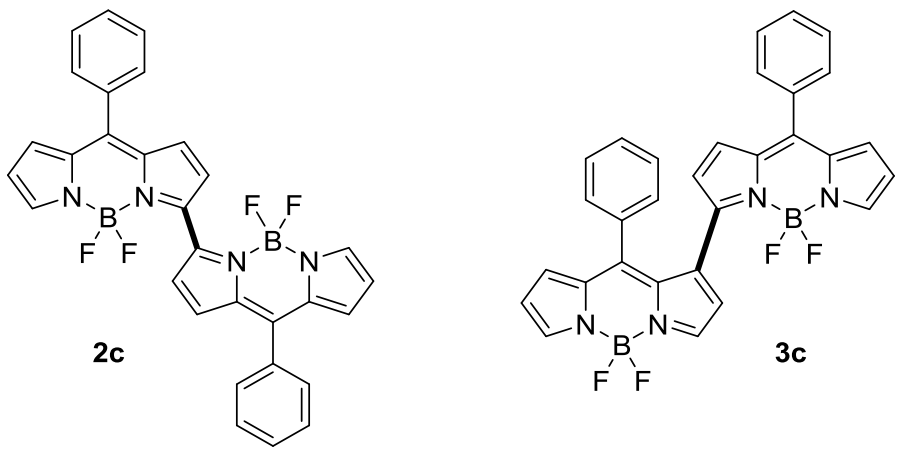

BODIPY dimers $\mathbf{2 c}$ and $\mathbf{3 c}$ were synthesized from BODIPY $1 \mathbf{c}(27 \mathrm{mg}, 0.1 \mathrm{mmol})$ using above general method:

BODIPY dimer $2 \mathbf{c}$ as a dark green solid in $56 \%$ yield $(15 \mathrm{mg})$ after silica column chromatography (silica, petroleum ether/dichloromethane: 2:1, v/v). ${ }^{1} \mathrm{H}$ NMR (500 $\left.\mathrm{MHz}, \mathrm{CDCl}_{3}\right) \delta: 7.92(\mathrm{~s}, 2 \mathrm{H}), 7.62-7.60(\mathrm{~m}, 6 \mathrm{H}), 7.56-7.53(\mathrm{~m}, 6 \mathrm{H}), 7.00(\mathrm{~d}, J=4.4$ $\mathrm{Hz}, 2 \mathrm{H}), 6.95(\mathrm{~d}, J=4.1 \mathrm{~Hz}, 2 \mathrm{H}), 6.57(\mathrm{~d}, J=4.0 \mathrm{~Hz}, 2 \mathrm{H}) .{ }^{13} \mathrm{C} \mathrm{NMR}\left(126 \mathrm{MHz}, \mathrm{CDCl}_{3}\right)$ $\delta: 148.1,146.3,144.7,137.7,135.4,134.1,131.4,131.3,130.8,130.7,128.5,123.9$, 119.3. HRMS calcd for $\mathrm{C}_{30} \mathrm{H}_{20} \mathrm{~B}_{2} \mathrm{~F}_{3} \mathrm{~N}_{4}[\mathrm{M}-\mathrm{F}]^{+}:$515.1826, found: 515.1847

BODIPY dimer 3c as a dark purple solid in $30 \%$ yield $(8 \mathrm{mg})$ after silica column chromatography (silica, petroleum ether/dichloromethane: 1:1, v/v). ${ }^{1} \mathrm{H}$ NMR (500 $\left.\mathrm{MHz}, \mathrm{CDCl}_{3}\right) \delta: 7.99(\mathrm{~d}, J=9.4 \mathrm{~Hz}, 2 \mathrm{H}), 7.88(\mathrm{~s}, 1 \mathrm{H}), 7.60-7.56(\mathrm{~m}, 1 \mathrm{H}), 7.54-7.51$ (m, 2H), 7.46-7.43 (m, 4H), $7.28(\mathrm{~d}, J=7.4 \mathrm{~Hz}, 1 \mathrm{H}), 7.17(\mathrm{~s}, 2 \mathrm{H}), 7.04(\mathrm{~s}, 1 \mathrm{H}), 6.87(\mathrm{t}$, $J=5.0 \mathrm{~Hz}, 2 \mathrm{H}), 6.56(\mathrm{~d}, J=4.0 \mathrm{~Hz}, 2 \mathrm{H}), 6.27(\mathrm{~d}, J=4.3 \mathrm{~Hz}, 1 \mathrm{H}), 5.53(\mathrm{~d}, J=4.3 \mathrm{~Hz}$, 1H). ${ }^{13} \mathrm{C}$ NMR $\left(126 \mathrm{MHz}, \mathrm{CDCl}_{3}\right) \delta: 152.6,147.9,145.3,143.1,142.5,136.3,136.0$, $135.8,134.5,134.0,133.9,132.8,132.6,130.9$, 130.6, 130.4, 130.2 , 130.1, 128.5, 127.8, 122.4, 122.0, 119.2, 118.4. HRMS calcd for $\mathrm{C}_{30} \mathrm{H}_{20} \mathrm{~B}_{2} \mathrm{~F}_{3} \mathrm{~N}_{4}[\mathrm{M}-\mathrm{F}]^{+}:$515.1826, found: 515.1851 

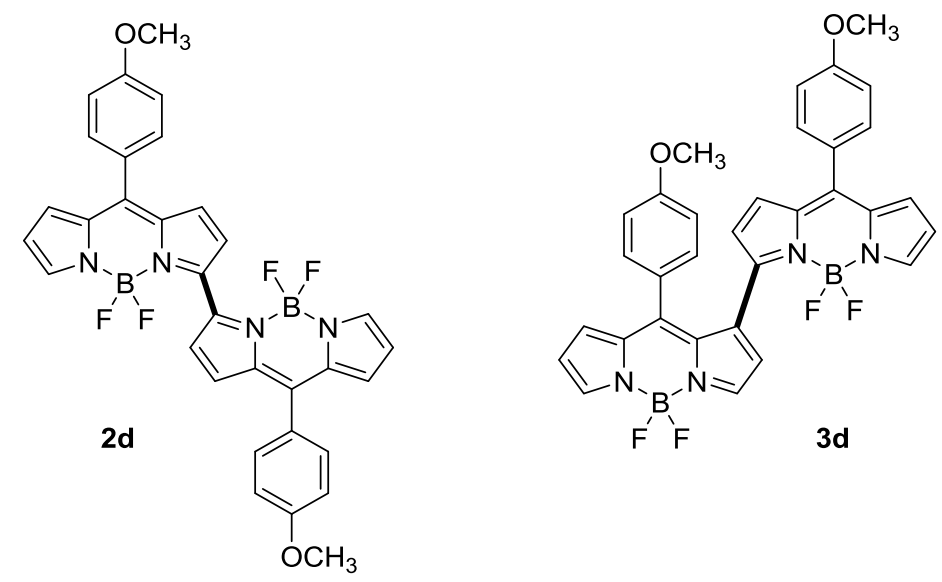

BODIPY dimers 2d and 3d were synthesized from BODIPY 1d (30 mg, $0.1 \mathrm{mmol})$ using above general method:

BODIPY dimer $2 \mathbf{d}$ as a dark blue solid in $36 \%$ yield (11 $\mathrm{mg}$ ) after silica column chromatography (silica, petroleum ether/dichloromethane: 2:1, v/v). ${ }^{1} \mathrm{H}$ NMR (300 $\left.\mathrm{MHz}, \mathrm{CDCl}_{3}\right) \delta$ : 7.89 (s, 2H), 7.60-7.52 (m, 6H), 7.08-6.97 (m, 8H), 6.57 (s, 2H), 3.93 (s, 6H). ${ }^{13} \mathrm{C}$ NMR $\left(126 \mathrm{MHz}, \mathrm{CDCl}_{3}\right) \delta: 162.1,147.7,146.3,143.9,137.8,135.3,132.6$, 131.1, 131.0, 126.7, 123.7, 118.9, 114.2, 55.6. HRMS calcd for $\mathrm{C}_{32} \mathrm{H}_{24} \mathrm{~B}_{2} \mathrm{~F}_{3} \mathrm{~N}_{4} \mathrm{O}_{2}[\mathrm{M}$ $\mathrm{F}^{+}:$575.2037, found: 575.2041 .

BODIPY dimer 3d as a dark purple solid in 19\% yield (5 mg) after silica column chromatography (silica, petroleum ether/dichloromethane: 2:1, v/v). ${ }^{1} \mathrm{H}$ NMR (500 $\left.\mathrm{MHz}, \mathrm{CDCl}_{3}\right) \delta: 7.97(\mathrm{~d}, J=8.5 \mathrm{~Hz}, 2 \mathrm{H}), 7.85(\mathrm{~s}, 1 \mathrm{H}), 7.45(\mathrm{~d}, J=8.6 \mathrm{~Hz}, 2 \mathrm{H}), 7.40$ $(\mathrm{d}, J=8.8 \mathrm{~Hz}, 2 \mathrm{H}), 7.04(\mathrm{~d}, J=8.5 \mathrm{~Hz}, 3 \mathrm{H}), 6.94(\mathrm{~d}, J=4.0 \mathrm{~Hz}, 1 \mathrm{H}), 6.90(\mathrm{~d}, J=4.0$ $\mathrm{Hz}, 1 \mathrm{H}), 6.60(\mathrm{~s}, 2 \mathrm{H}), 6.56(\mathrm{~d}, J=3.7 \mathrm{~Hz}, 2 \mathrm{H}), 6.39(\mathrm{~d}, J=4.3 \mathrm{~Hz}, 1 \mathrm{H}), 5.54(\mathrm{~d}, J=4.3$ $\mathrm{Hz}, 1 \mathrm{H}), 3.92(\mathrm{~s}, 3 \mathrm{H}), 3.68(\mathrm{~s}, 3 \mathrm{H}) .{ }^{13} \mathrm{C} \mathrm{NMR}\left(126 \mathrm{MHz}, \mathrm{CDCl}_{3}\right) \delta: 161.9,161.8,152.5$, 148.0, 145.3, 144.6, 142.2, 142.0, 136.1, 135.8, 134.3, 132.8, 132.4, 132.2, 129.9, 129.7, 126.5, 126.5, 122.5, 121.8, 119.0, 118.0, 114.2, 113.4, 55.7, 55.2. HRMS calcd for $\mathrm{C}_{32} \mathrm{H}_{24} \mathrm{~B}_{2} \mathrm{~F}_{3} \mathrm{~N}_{4} \mathrm{O}_{2}[\mathrm{M}-\mathrm{F}]^{+}:$575.2037, found : 575.2035 . 

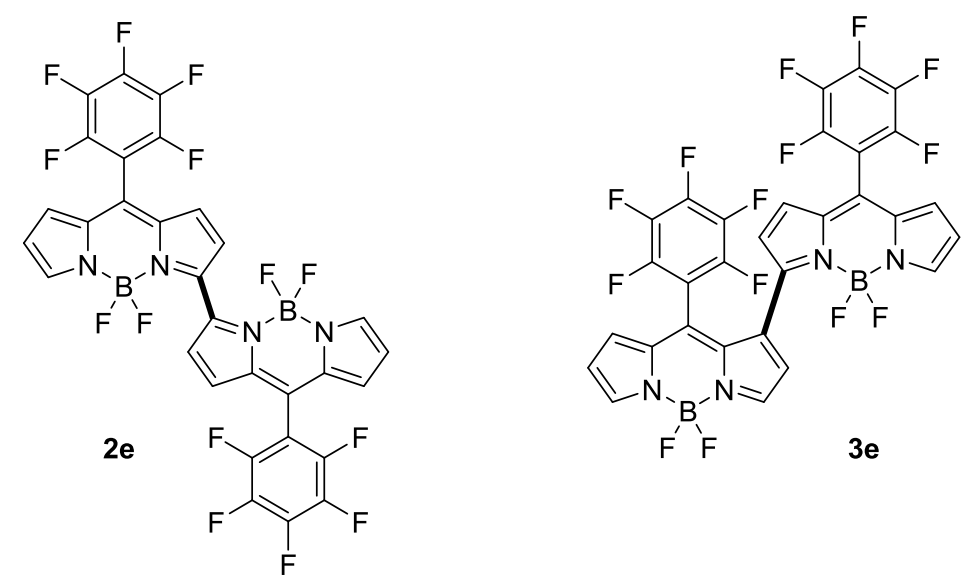

BODIPY dimers 2e and 3e were synthesized from BODIPY 1 e (36 mg, $0.1 \mathrm{mmol})$ using above general method:

BODIPY dimer 2e as a dark yellow solid in $15 \%$ yield $(5 \mathrm{mg})$ after silica column chromatography (silica, petroleum ether/dichloromethane: 2:1, v/v). ${ }^{1} \mathrm{H}$ NMR (500 $\left.\mathrm{MHz}, \mathrm{CDCl}_{3}\right) \delta: 7.99$ (s, 2H), $7.61(\mathrm{~d}, J=4.4 \mathrm{~Hz}, 2 \mathrm{H}), 6.85$ (d, $\left.J=4.3 \mathrm{~Hz}, 4 \mathrm{H}\right), 6.63$ $(\mathrm{d}, J=3.9 \mathrm{~Hz}, 2 \mathrm{H}) .{ }^{13} \mathrm{C} \mathrm{NMR}\left(126 \mathrm{MHz}, \mathrm{CDCl}_{3}\right) \delta: 149.0,147.9,145.7,143.7,141.6$, 139.0, 137.7, 136.9, 135.8, 130.6, 129.7, 128.1, 125.2, 121.0, 108.1. HRMS calcd for $\mathrm{C}_{30} \mathrm{H}_{10} \mathrm{~B}_{2} \mathrm{~F}_{13} \mathrm{~N}_{4}[\mathrm{M}-\mathrm{F}]^{+}$: 695.0884, found 695.0881.

BODIPY dimer $3 \mathbf{e}$ as a purple solid in $13 \%$ yield $(4 \mathrm{mg})$ after silica column chromatography (silica, petroleum ether/dichloromethane: 1:1, v/v). ${ }^{1} \mathrm{H}$ NMR (500 $\left.\mathrm{MHz}, \mathrm{CDCl}_{3}\right) \delta: 8.07$ (s, 1H), $8.02(\mathrm{~s}, 1 \mathrm{H}), 7.98$ (s, 1H), 6.99 (s, 1H), 6.84 (d, J = 4.2 $\mathrm{Hz}, 1 \mathrm{H}), 6.79$ (d, $J=4.2 \mathrm{~Hz}, 1 \mathrm{H}), 6.63(\mathrm{~d}, J=3.9 \mathrm{~Hz}, 1 \mathrm{H}), 6.61(\mathrm{~d}, J=4.0 \mathrm{~Hz}, 1 \mathrm{H})$, $6.50(\mathrm{~d}, J=4.1 \mathrm{~Hz}, 1 \mathrm{H}), 6.10(\mathrm{~d}, J=4.2 \mathrm{~Hz}, 1 \mathrm{H}) .{ }^{13} \mathrm{C} \mathrm{NMR}\left(126 \mathrm{MHz}, \mathrm{CDCl}_{3}\right) \delta: 150.9$, 148.5, 148.2, 144.5, 143.7, 139.0, 138.5, 136.9, 136.4, 135.4, 135.2, 132.5, 131.7, 131.1, 129.6, 128.4, 127.8, 122.4, 121.8, 121.1, 120.7, 108.3, 107.7. HRMS calcd for $\mathrm{C}_{30} \mathrm{H}_{10} \mathrm{~B}_{2} \mathrm{~F}_{13} \mathrm{~N}_{4}[\mathrm{M}-\mathrm{F}]^{+}: 695.0884$ found 695.0862. 

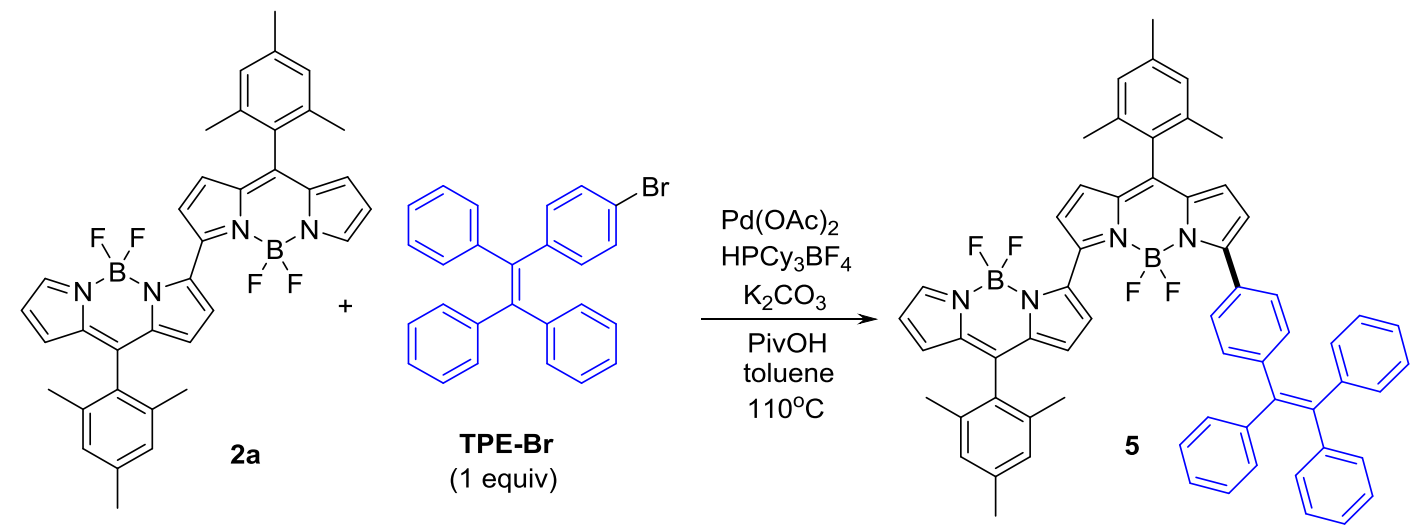

Syhthesis of BODIPY 5: BODIPY $2 \mathbf{a}(0.15 \mathrm{mmol}, 100 \mathrm{mg})$, TPE-Br $(0.15 \mathrm{mmol}, 66$ $\mathrm{mg}), \mathrm{Pd}(\mathrm{OAc})_{2}(0.015 \mathrm{mmol}, 5 \mathrm{~mol} \%), \mathrm{PCy}_{3} \mathrm{HBF}_{4}(0.03 \mathrm{mmol}, 10 \mathrm{~mol} \%), \mathrm{K}_{2} \mathrm{CO}_{3}(3$ equiv, $0.9 \mathrm{mmol})$ and $\mathrm{Piv}(\mathrm{OH})(0.09 \mathrm{mmol}, 30 \mathrm{~mol} \%)$ were dissolved in $3 \mathrm{~mL}$ toluene. The reaction mixture was stirred at $110{ }^{\circ} \mathrm{C}$ for $12 \mathrm{~h}$ under nitrogen. Upon completion, the reaction mixture was cooled to room temperature and was poured into dichloromethane $(100 \mathrm{~mL})$, washed three times with water $(100 \mathrm{~mL})$. The crude product was then purified by column chromatography on silica gel using petroleum ether/ethyl acetate $(50: 1 \mathrm{v} / \mathrm{v})$ as eluent to give product 5 as green solid in $30 \%$ yield $(45 \mathrm{mg}) .{ }^{1} \mathrm{H}$ NMR (400 MHz, $\left.\mathrm{CDCl}_{3}\right) \delta: 7.83(\mathrm{~s}, 1 \mathrm{H}), 7.72(\mathrm{~d}, J=8.5 \mathrm{~Hz}, 2 \mathrm{H}), 7.51(\mathrm{~d}, J=4.4 \mathrm{~Hz}$, $1 \mathrm{H}), 7.44(\mathrm{~d}, J=4.5 \mathrm{~Hz}, 1 \mathrm{H}), 7.12-7.01(\mathrm{~m}, 17 \mathrm{H}), 6.97(\mathrm{~d}, J=2.4 \mathrm{~Hz}, 4 \mathrm{H}), 6.69$ (d, $J$ $=4.4 \mathrm{~Hz}, 2 \mathrm{H}), 6.63(\mathrm{dd}, J=10.4,4.3 \mathrm{~Hz}, 3 \mathrm{H}), 6.45(\mathrm{dd}, J=4.1,1.7 \mathrm{~Hz}, 1 \mathrm{H}), 2.38(\mathrm{~d}$, $J=3.0 \mathrm{~Hz}, 6 \mathrm{H}), 2.17(\mathrm{~d}, J=4.3 \mathrm{~Hz}, 12 \mathrm{H}) ;{ }^{13} \mathrm{C} \mathrm{NMR}\left(101 \mathrm{MHz}, \mathrm{CDCl}_{3}\right) \delta: 160.9,149.4$, $145.7,145.6,145.3,143.4,143.3,143.3,143.2$, 142.13, 140.3, 138.7, 138.7, 138.4, $138.1,137.4,136.8,136.6,135.3,131.5,131.3,130.9$, 130.2, 130.1, 129.8, 128.0, 128.9, $128.2,127.8,127.7,127.6,127.4,126.7,126.6,124.5,124.0,123.9,122.4,118.6,21.2$, 20.3, 20.2. HRMS calcd. For $\mathrm{C}_{62} \mathrm{H}_{50} \mathrm{~B}_{2} \mathrm{~F}_{4} \mathrm{~N}_{4}[\mathrm{M}]^{+}:$948.415228, found 948.416298 . 

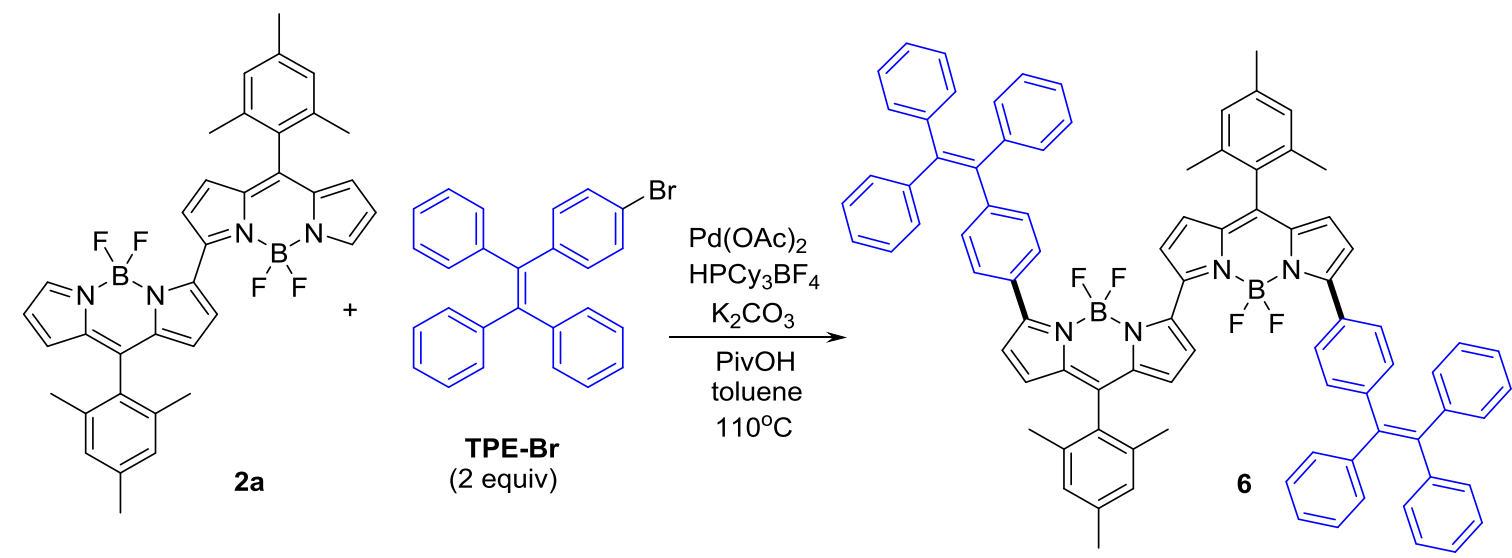

Syhthesis of BODIPY 6: BODIPY 2a $(0.15 \mathrm{mmol}, 100 \mathrm{mg})$, TPE-Br $(0.3 \mathrm{mmol}, 125$ $\mathrm{mg}), \mathrm{Pd}(\mathrm{OAc})_{2}(0.015 \mathrm{mmol}, 5 \mathrm{~mol} \%), \mathrm{PCy}_{3} \mathrm{HBF}_{4}(0.03 \mathrm{mmol}, 10 \mathrm{~mol} \%), \mathrm{K}_{2} \mathrm{CO}_{3}(3$ equiv, $0.9 \mathrm{mmol})$ and $\mathrm{Piv}(\mathrm{OH})(0.09 \mathrm{mmol}, 30 \mathrm{~mol} \%)$ were dissolved in $3 \mathrm{~mL}$ toluene. The reaction mixture was stirred at $110{ }^{\circ} \mathrm{C}$ for $12 \mathrm{~h}$ under nitrogen. Upon completion, the reaction mixture was cooled to room temperature and was poured into dichloromethane $(100 \mathrm{~mL})$, washed three times with water $(100 \mathrm{~mL})$. The crude product was then purified by column chromatography on silica gel using petroleum ether/ethyl acetate $(100: 1 \mathrm{v} / \mathrm{v})$ as eluent to give products 6 as red solid in $22 \%$ yield $(42 \mathrm{mg}) .{ }^{1} \mathrm{H}$ NMR (400 MHz, $\left.\mathrm{CDCl}_{3}\right) \delta: 7.70(\mathrm{~d}, J=8.1 \mathrm{~Hz}, 4 \mathrm{H}), 7.42(\mathrm{~s}, 2 \mathrm{H}), 7.12-7.01(\mathrm{~m}, 34 \mathrm{H})$, $6.97(\mathrm{~s}, 4 \mathrm{H}), 6.64(\mathrm{~d}, J=4.2 \mathrm{~Hz}, 2 \mathrm{H}), 6.63-6.55(\mathrm{~m}, 4 \mathrm{H}), 2.37(\mathrm{~s}, 6 \mathrm{H}), 2.18(\mathrm{~s}, 12 \mathrm{H})$; ${ }^{13} \mathrm{C} \mathrm{NMR}\left(101 \mathrm{MHz}, \mathrm{CDCl}_{3}\right) \delta: 159.8,147.0,145.3,143.6,143.4,143.3,142.6,141.9$, $140.4,138.5,137.9,137.3,136.9,131.5,131.3,131.3,131.2,130.4,130.1,130.0,129.0$, 128.1, 127.8, 127.7, 127.6, 126.7, 126.5, 126.5, 124.4, 121.8, 21.2, 20.3. HRMS calcd. For $\mathrm{C}_{88} \mathrm{H}_{68} \mathrm{~B}_{2} \mathrm{~F}_{4} \mathrm{~N}_{4}[\mathrm{M}]^{+}:$1278.556078, found 1278.558553 . 

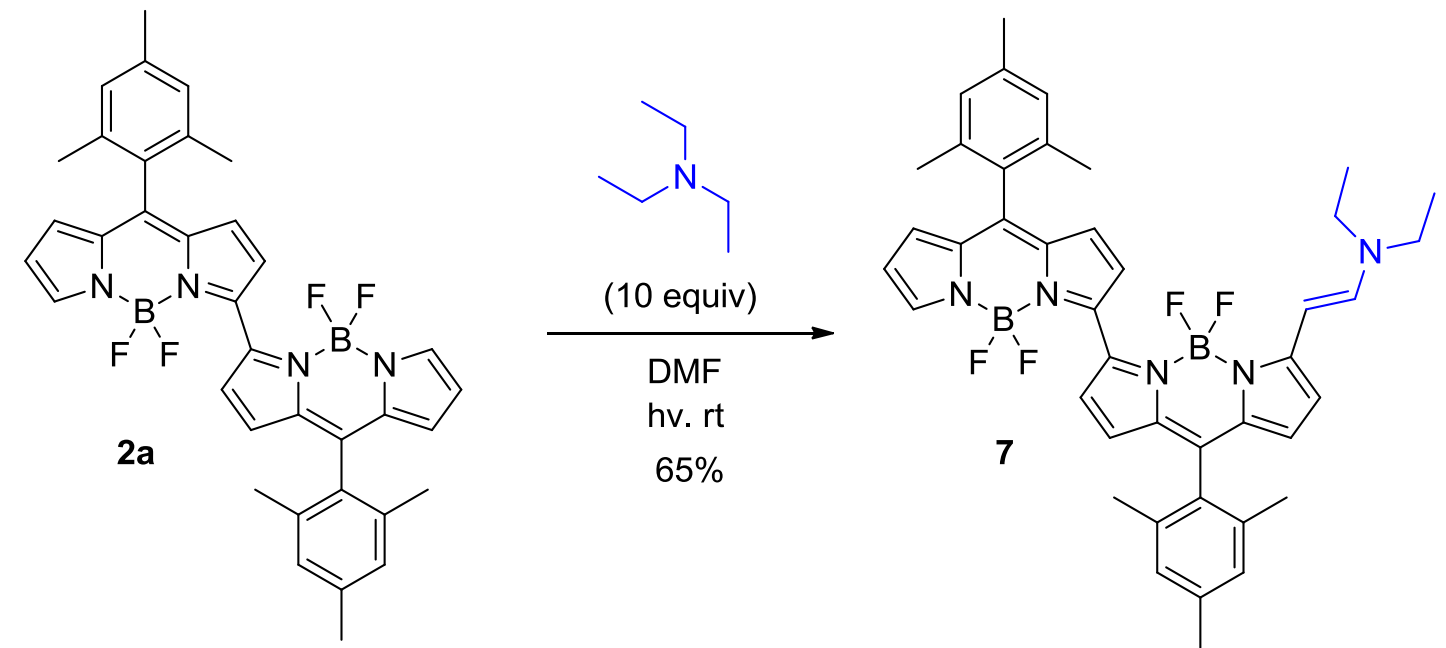

Synthesis of BODIPY dimer 7 from 2a: To $2.0 \mathrm{~mL} \mathrm{DMF}$ in a $10 \mathrm{~mL}$ reaction tube was added BODIPY 2a $(0.1 \mathrm{mmol}, 62 \mathrm{mg})$, triethylamine $(1.0 \mathrm{mmol}, 140 \mu \mathrm{L})$. The mixture was irradiated with $10 \mathrm{~W}$ white LED at $\mathrm{rt}$ for $24 \mathrm{~h}$. TLC was used to follow the reaction. Upon the completion of the reaction, the mixture was poured into aqueous $\mathrm{NaHCO}_{3}$ and was extracted with dichloromethane $(30 \mathrm{~mL} \times 3)$. Organic layers were combined, dried over anhydrous $\mathrm{Na}_{2} \mathrm{SO}_{4}$, and organic solvent was removed under vacuum. The crude product was purified by column chromatography (silica, petroleum ether/ethyl acetate $=1: 1, \mathrm{v} / \mathrm{v})$ on silica to afford the desired BODIPY 7 as dark blue solids in $65 \%$ yield $(46 \mathrm{mg}) .{ }^{1} \mathrm{H}$ NMR $\left(500 \mathrm{MHz}, \mathrm{CDCl}_{3}\right) \delta$ : 7.68-7.66 (m, 3H), $7.36(\mathrm{~d}, J=13.0 \mathrm{~Hz}$, 1H), $6.94(\mathrm{~d}, J=6.7 \mathrm{~Hz}, 4 \mathrm{H}), 6.68(\mathrm{~d}, J=3.7 \mathrm{~Hz}, 2 \mathrm{H}), 6.53(\mathrm{~d}, J=2.5 \mathrm{~Hz}, 1 \mathrm{H}), 6.44$ (s, 1H), $6.35(\mathrm{~s}, 1 \mathrm{H}), 6.20(\mathrm{~s}, 1 \mathrm{H}), 6.10(\mathrm{~d}, J=6.5 \mathrm{~Hz}, 1 \mathrm{H}), 3.38(\mathrm{~s}, 4 \mathrm{H}), 2.35$ (d, $J=$ $5.8 \mathrm{~Hz}, 6 \mathrm{H}), 2.16(\mathrm{~s}, 6 \mathrm{H}), 2.13(\mathrm{~s}, 6 \mathrm{H}), 1.24(\mathrm{t}, J=7.1 \mathrm{~Hz}, 6 \mathrm{H}) .{ }^{13} \mathrm{C} \mathrm{NMR}(126 \mathrm{MHz}$, $\left.\mathrm{CDCl}_{3}\right) \delta: 164.0,153.9,150.8,141.2,139.1,138.6,138.4,138.0,137.7,137.2,134.2$, $131.4,131.1,131.0,130.8,128.3,128.2,125.1,122.9,122.8,122.7,119.8,119.5,116.6$, 91.4, 51.3, 43.3, 21.3, 20.4, 15.1, 12.2. HRMS calcd for $\mathrm{C}_{42} \mathrm{H}_{44} \mathrm{~B}_{2} \mathrm{~F}_{4} \mathrm{~N}_{5}[\mathrm{M}+\mathrm{H}]^{+}$: 716.3719, found: 716.3701 . 

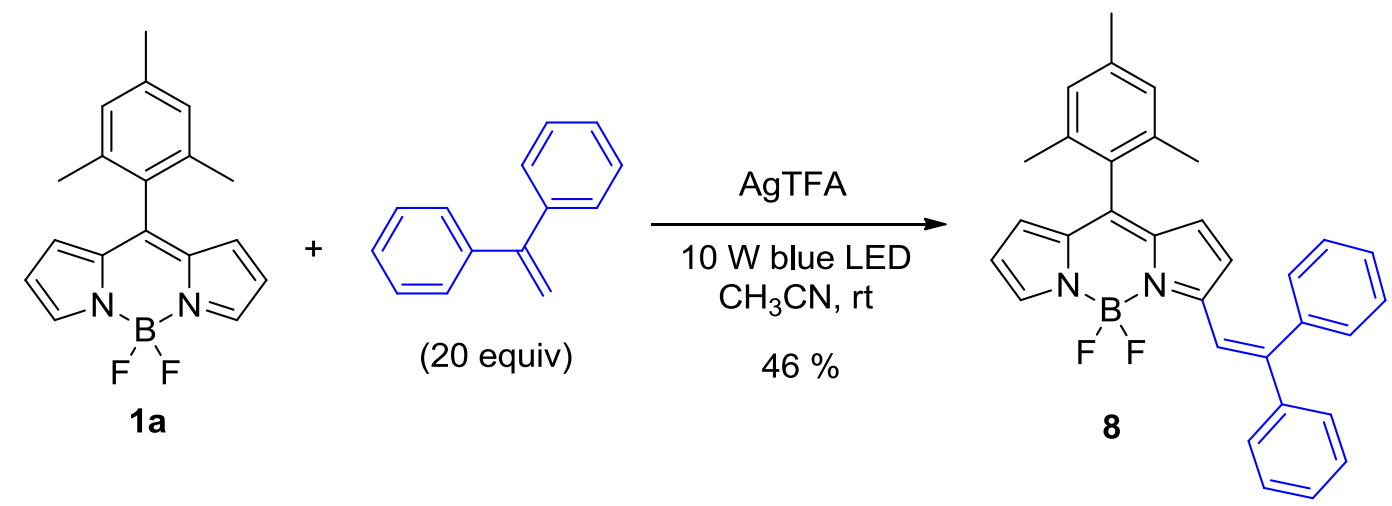

Syhthesis of 8: To a solution of $\mathbf{1 a}(0.1 \mathrm{mmol}, 31 \mathrm{mg})$ and 1,1-diphenylethylene (20 equiv, $344 \mu \mathrm{L}$ ) in distilled acetonitrile ( $1 \mathrm{~mL}$ ) was added AgTFA ( $88 \mathrm{mg}$, 4 equiv, 0.4 mmol). The reaction mixture was stirred at room tempreture for $12 \mathrm{~h}$ under the irridiation of a $10 \mathrm{~W}$ blue LED $(450 \mathrm{~nm})$. The reaction mixture was then evaporated under vacuum. The residue was then purified by column chromatography on silica gel using petroleum ether/dichloromethane (3/1, v/v) as eluent to give product $\mathbf{8}$ as red solid in $46 \%$ yield $(22 \mathrm{mg}) .{ }^{1} \mathrm{H}$ NMR $\left(500 \mathrm{MHz}, \mathrm{CDCl}_{3}\right) \delta: 7.80(\mathrm{~d}, J=5.7 \mathrm{~Hz}, 2 \mathrm{H}), 7.48-$ $7.40(\mathrm{~m}, 5 \mathrm{H}), 7.38-7.33(\mathrm{~m}, 3 \mathrm{H}), 7.32-7.30(\mathrm{~m}, 2 \mathrm{H}), 6.90(\mathrm{~s}, 2 \mathrm{H}), 6.50(\mathrm{t}, J=6.2 \mathrm{~Hz}$, $1 \mathrm{H}), 6.42-6.40(\mathrm{~m}, 1 \mathrm{H}), 6.31(\mathrm{~d}, J=4.7 \mathrm{~Hz}, 1 \mathrm{H}), 5.36(\mathrm{~d}, J=4.7 \mathrm{~Hz}, 1 \mathrm{H}), 2.32(\mathrm{~s}, 3 \mathrm{H})$, 2.07 (s, 6H). ${ }^{13} \mathrm{C} \mathrm{NMR}\left(126 \mathrm{MHz}, \mathrm{CDCl}_{3}\right) \delta: 157.0,151.4,142.8,141.5,140.6,139.9$, 139.7, 139.6, 138.4, 136.6, 134.7, 130.1, 129.2, 129.0, 128.7, 128.6, 128.3, 128.2, 121.1, 118.4, 117.3, 21.1, 20.0. HRMS calcd for $\mathrm{C}_{32} \mathrm{H}_{27} \mathrm{~B}_{2} \mathrm{FN}_{2}[\mathrm{M}-\mathrm{F}]^{+}$: 469.2251, found: 469.2221 . 

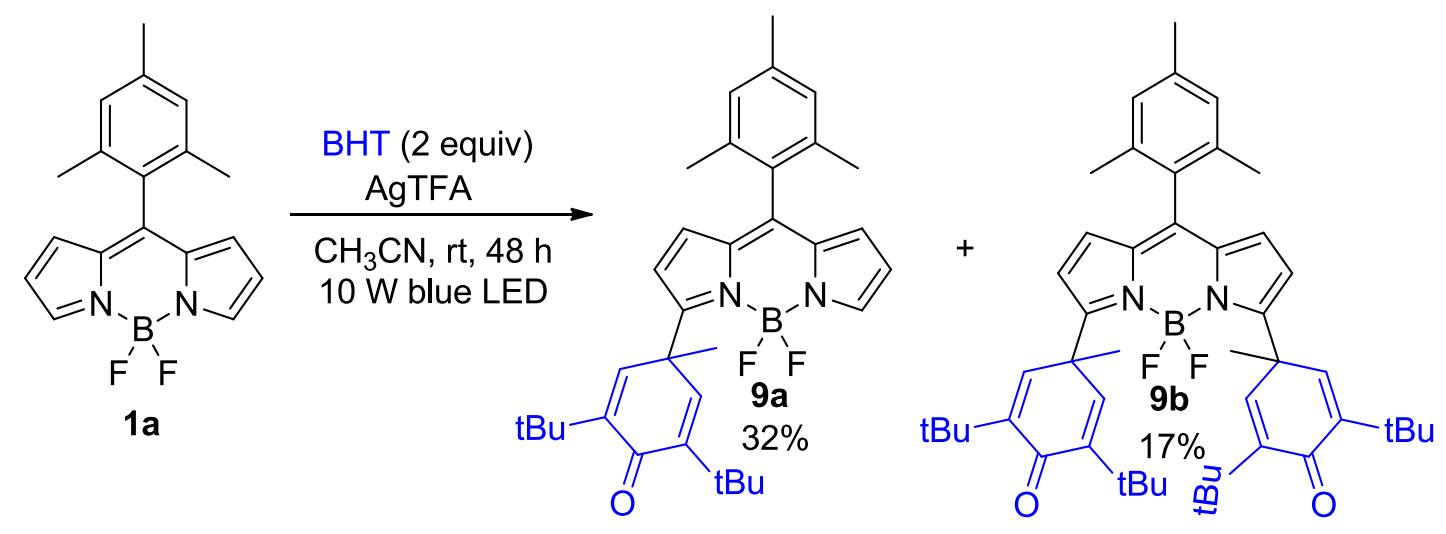

Syhthesis of BODIPYs 9a and 9b: To a solution of $\mathbf{1 a}(0.1 \mathrm{mmol}, 31 \mathrm{mg})$ and 2,6-tertbutyl-4-methylphenol (BHT) (44 mg, 2 equiv, $0.2 \mathrm{mmol})$ in distilled acetonitrile (1 mL) was added AgTFA ( $88 \mathrm{mg}, 4$ equiv, $0.4 \mathrm{mmol}$ ). The reaction mixture was stirred at room tempreture for $12 \mathrm{~h}$ under the irridiation of a $10 \mathrm{~W}$ blue LED $(450 \mathrm{~nm})$. The reaction mixture was evaporated under vacuum. The residue was then purified by column chromatography on silica gel using petroleum ether /dichloromethane $(6 / 1, \mathrm{v} / \mathrm{v})$ as eluent to give products $9 \mathbf{a}$ as yellow solid in $32 \%$ yield $(17 \mathrm{mg})$ and $\mathbf{9 b}$ as orange solid in $17 \%$ yield $(13 \mathrm{mg})$.

9a: ${ }^{1} \mathrm{H}$ NMR $\left(500 \mathrm{MHz}, \mathrm{CDCl}_{3}\right) \delta: 7.85(\mathrm{~s}, 1 \mathrm{H}), 6.97$ (s, 2H), 6.94 (s, 2H), $6.62(\mathrm{~d}, J$ $=4.1 \mathrm{~Hz}, 1 \mathrm{H}), 6.59(\mathrm{~d}, J=4.3 \mathrm{~Hz}, 1 \mathrm{H}), 6.46(\mathrm{~d}, J=4.0 \mathrm{~Hz}, 1 \mathrm{H}), 6.29(\mathrm{~d}, J=4.3 \mathrm{~Hz}$, $1 \mathrm{H}), 2.36(\mathrm{~s}, 3 \mathrm{H}), 2.10(\mathrm{~s}, 6 \mathrm{H}), 1.81(\mathrm{~s}, 3 \mathrm{H}), 1.28(\mathrm{~s}, 18 \mathrm{H}) .{ }^{13} \mathrm{C} \mathrm{NMR}\left(126 \mathrm{MHz}, \mathrm{CDCl}_{3}\right)$ $\delta: 186.4,163.2,146.7,145.6,143.8,143.7,139.0,137.7,136.7,134.7,131.0,130.1$, 129.2, 128.3, 118.9, 118.8, 42.8, 35.1, 29.6, 26.9, 21.3, 20.2. HRMS calcd for $\mathrm{C}_{33} \mathrm{H}_{40} \mathrm{BF}_{2} \mathrm{~N}_{2} \mathrm{O}[\mathrm{M}+\mathrm{H}]^{+}:$529.3202, found: 529.3232.

9b: ${ }^{1} \mathrm{H}$ NMR $\left(500 \mathrm{MHz}, \mathrm{CDCl}_{3}\right) \delta: 6.95(\mathrm{~s}, 4 \mathrm{H}), 6.93(\mathrm{~s}, 2 \mathrm{H}), 6.51(\mathrm{~d}, J=4.3 \mathrm{~Hz}, 2 \mathrm{H})$, $6.28(\mathrm{~d}, J=4.3 \mathrm{~Hz}, 2 \mathrm{H}), 2.35(\mathrm{~s}, 3 \mathrm{H}), 2.10(\mathrm{~s}, 6 \mathrm{H}), 1.79$ (s, 6H), $1.26(\mathrm{~s}, 36 \mathrm{H}),{ }^{13} \mathrm{C}$ NMR (126 MHz, $\left.\mathrm{CDCl}_{3}\right) \delta: 186.4,162.2,145.3,144.4,138.9,136.9,130.3,129.7$, 128.3, 119.5, 43.0, 35.1, 29.67, 26.7, 21.4, 20.3. HRMS calcd for $\mathrm{C}_{48} \mathrm{H}_{61} \mathrm{BFN}_{2} \mathrm{O}_{2}[\mathrm{M}-$ F] ${ }^{+}:$727.4810, found: 727.4793 . 


\section{Crystal data}
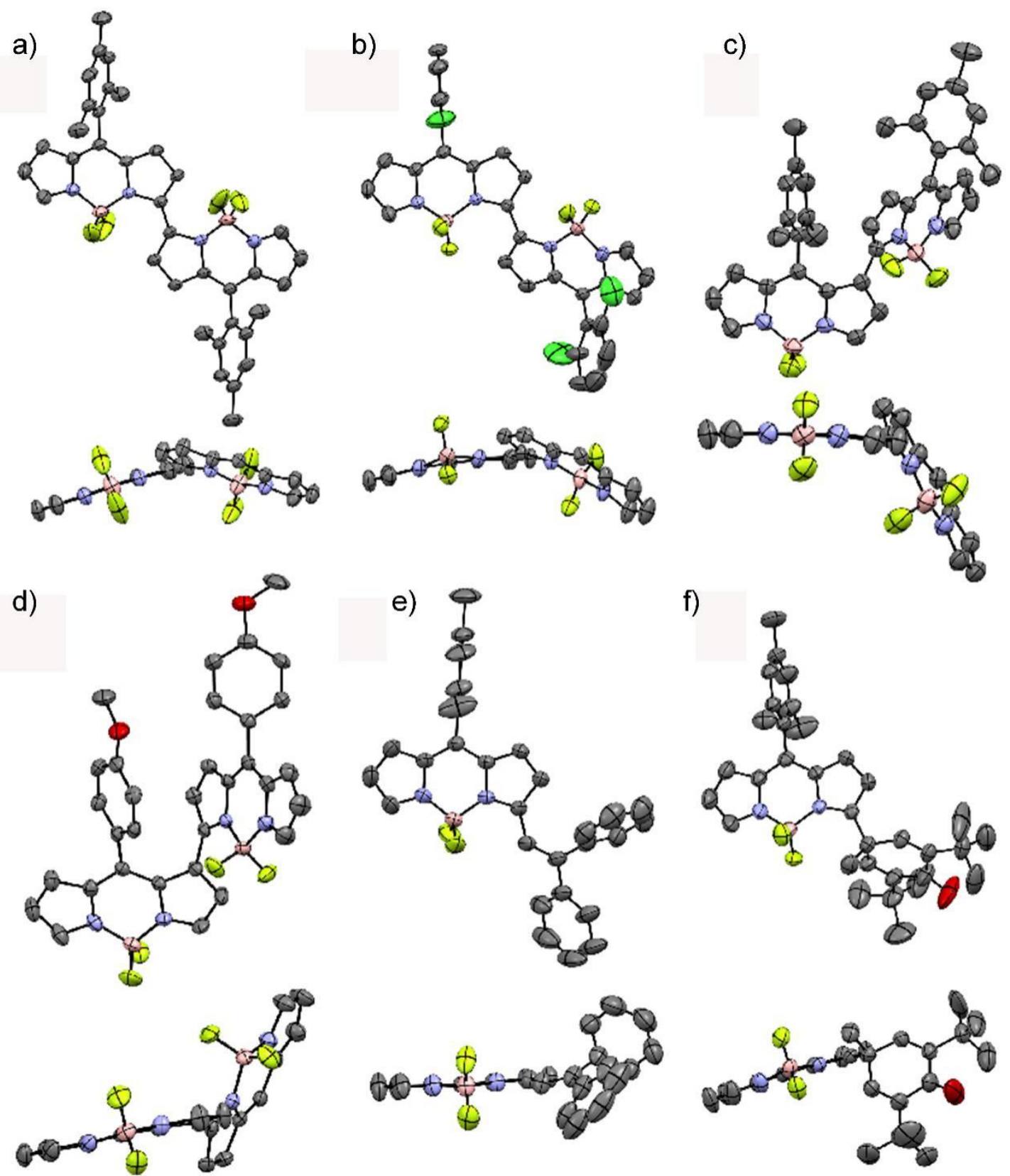

Figure S4. X-ray crystal structures (top and side views) of dimers $\mathbf{2 a}$ (a), 3a (b), 2b (c), 3d (d), 8 (e) and 9a (f): C, gray; N, blue; B, pink; F, bright green; O, red. Hydrogen atoms were removed for clarity. Aryl substituents were omitted in side views. Thermal ellipsoids are shown at the 50\% probability level. 
Table S2. Selected geometrical parameters of compounds $2,3,5,8$ and 9a obtained from crystallography

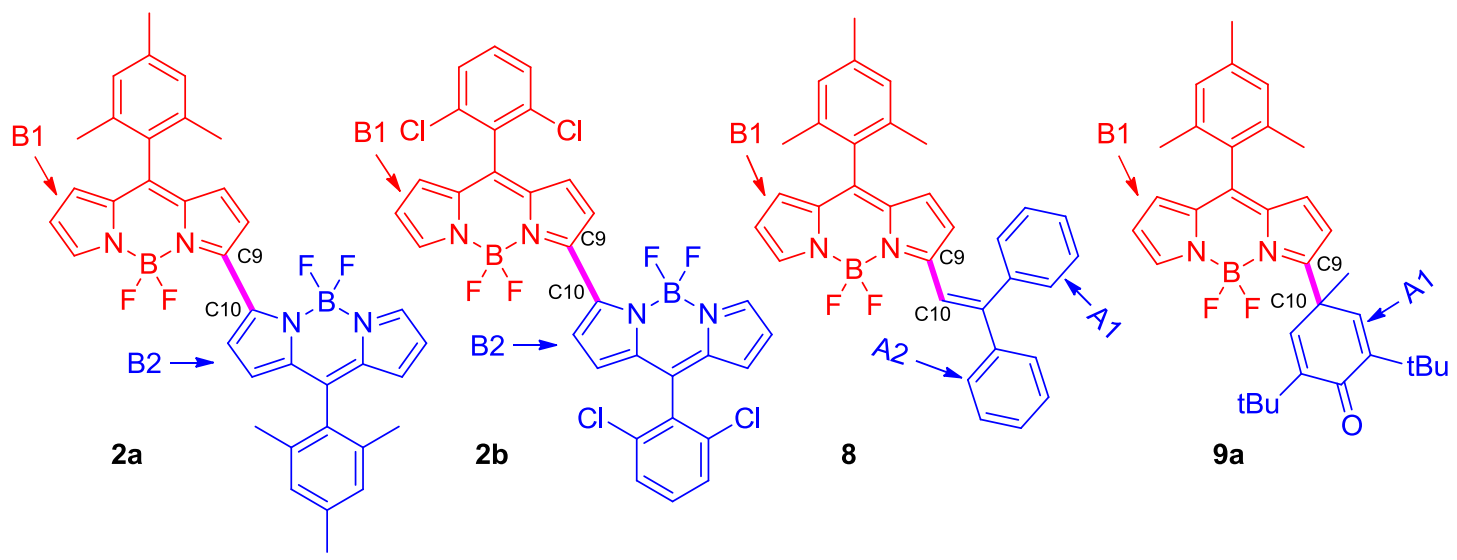

\begin{tabular}{|c|c|c|c|c|}
\hline & $\mathbf{2 a}$ & $\mathbf{2 b}$ & 8 & $9 \mathbf{a}$ \\
\hline the $\mathrm{C}_{9}-\mathrm{C}_{10}$ bond distances $(\AA)$ & $1.38(5)$ & $1.45(5)$ & $1.45(1)$ & $1.52(5)$ \\
\hline \multirow[t]{4}{*}{ the B-N bond distances $(\AA)$} & $1.53(4)$ & $1.56(1)$ & $1.53(0)$ & $1.56(9)$ \\
\hline & $1.53(6)$ & $1.55(6)$ & $1.55(2)$ & $1.55(5)$ \\
\hline & $1.53(4)$ & $1.56(3)$ & & \\
\hline & $1.53(6)$ & $1.54(2)$ & & \\
\hline $\begin{array}{l}\text { dihedral angles between meso-aryl and } \\
\text { dipyrrin core in B1 (deg) }\end{array}$ & $82.77(4)$ & $89.42(7)$ & $84.06(3)$ & $86.15(6)$ \\
\hline $\begin{array}{c}\text { dihedral angles between meso-aryl and } \\
\text { dipyrrin core in B2 (deg) }\end{array}$ & $82.77(4)$ & $80.85(2)$ & I & / \\
\hline $\begin{array}{l}\text { dihedral angles of two dipyrrin cores in B1 } \\
\text { and B2 (deg) }\end{array}$ & $40.61(1)$ & $41.73(1)$ & I & / \\
\hline the average and maximum deviations of & 0.030 & 0.097 & 0.028 & 0.027 \\
\hline $\begin{array}{l}\text { the eleven atoms from the mean plane of } \\
\text { dipyrrin }\left(\mathrm{C}_{9} \mathrm{~N}_{2}\right) \text { core in } \mathrm{B} 1(\AA)\end{array}$ & 0.058 & 0.19 & 0.053 & 0.054 \\
\hline the average and maximum deviations of & 0.030 & 0.096 & / & I \\
\hline $\begin{array}{l}\text { the eleven atoms from the mean plane of } \\
\text { dipyrrin }\left(\mathrm{C}_{9} \mathrm{~N}_{2}\right) \text { core in } \mathrm{B} 2(\AA)\end{array}$ & 0.058 & 0.18 & & \\
\hline $\begin{array}{l}\text { dihedral angles of two benzene rings A1 } \\
\text { and A2 }\end{array}$ & I & I & $72.64(1)$ & I \\
\hline $\begin{array}{c}\text { dihedral angles of } \mathrm{A} 1 \text { and } \mathrm{B} 1 \text { and/or } \mathrm{A} 2 \\
\text { and B1 rings }\end{array}$ & I & 1 & $\begin{array}{l}67.00(1) \\
49.41(8)\end{array}$ & $62.48(4)$ \\
\hline
\end{tabular}




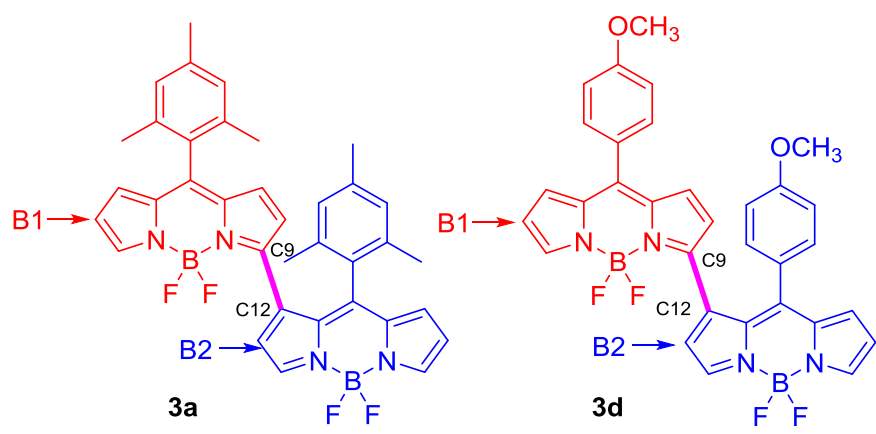

\begin{tabular}{|c|c|c|}
\hline & 3a & 3d \\
\hline the $\mathrm{C}_{9}-\mathrm{C}_{12}$ bond distances $(\AA)$ & $1.46(3)$ & $1.46(6)$ \\
\hline the B-N bond distances $(\AA)$ & $1.53(3)$ & $1.54(3)$ \\
& $1.56(4)$ & $1.55(7)$ \\
$1.54(8)$ & $1.54(3)$ \\
$1.53(2)$ & $54(4)$ \\
\hline $\begin{array}{c}\text { dihedral angles between meso-aryl and } \\
\text { dipyrrin core in B1 (deg) }\end{array}$ & $83.66(5)$ & $53.27(6)$ \\
\hline $\begin{array}{c}\text { dihedral angles between } \text { meso-aryl and } \\
\text { dipyrrin core in B2 (deg) }\end{array}$ & $77.13(6)$ & $53.03(6)$ \\
\hline $\begin{array}{c}\text { dihedral angles of two dipyrrin cores in } \mathrm{B} 1 \\
\text { and } \mathrm{B} 2(\mathrm{deg})\end{array}$ & $62.56(8)$ & $57.89(3)$ \\
\hline $\begin{array}{c}\text { the average and maximum deviations of the } \\
\text { eleven atoms from the mean plane of } \\
\text { dipyrrin }\left(\mathrm{C}_{9} \mathrm{~N}_{2}\right) \text { core in } \mathrm{B} 1(\AA)\end{array}$ & $0.032 / 0.066$ & $0.013 / 0.025$ \\
\hline $\begin{array}{c}\text { the average and maximum deviations of the } \\
\text { eleven atoms from the mean plane of } \\
\text { dipyrrin }\left(\mathrm{C}_{9} \mathrm{~N}_{2}\right) \text { core in } \mathrm{B} 2(\AA)\end{array}$ & $0.045 / 0.092$ & $0.035 / 0.068$ \\
\hline
\end{tabular}


Table S3. Crystal experimental details.

\begin{tabular}{|c|c|c|c|}
\hline Crystal data & $\mathbf{2 a}$ & $\mathbf{3 a}$ & 9a \\
\hline Chemical formula & $\mathrm{C}_{36} \mathrm{H}_{32} \mathrm{~B}_{2} \mathrm{~F}_{4} \mathrm{~N}_{4}$ & $\mathrm{C}_{36} \mathrm{H}_{32} \mathrm{~B}_{2} \mathrm{~F}_{4} \mathrm{~N}_{4}$ & $\mathrm{C}_{33} \mathrm{H}_{39} \mathrm{BF}_{2} \mathrm{~N}_{2} \mathrm{O}$ \\
\hline$M_{\mathrm{r}}$ & 618.28 & 618.28 & 528.47 \\
\hline $\begin{array}{l}\text { Crystal system, space } \\
\text { group }\end{array}$ & $\begin{array}{c}\text { monoclinic, } \\
P 2 / c\end{array}$ & $\begin{array}{l}\text { monoclinic, } \\
\qquad P 2_{1} / n\end{array}$ & $\begin{array}{l}\text { monoclinic, } \\
C 2 / c\end{array}$ \\
\hline Temperature (K) & 293.15 & 293.15 & 273.15 \\
\hline$a, b, c(\AA)$ & $\begin{array}{c}9.3235(10), \\
10.9925(12), \\
15.5891(17)\end{array}$ & $\begin{array}{c}12.6694(17), \\
15.300(2), \\
17.631(3)\end{array}$ & $\begin{array}{c}26.285(5), 11.639(3), \\
23.133(4)\end{array}$ \\
\hline$\alpha, \beta, \gamma\left(^{\circ}\right)$ & 90, 103.3570(10), 90 & $\begin{array}{c}90, \quad 110.947(2), \\
90\end{array}$ & $\begin{array}{l}\text { 90, } 120.38(7), \\
90\end{array}$ \\
\hline$V\left(\AA^{3}\right)$ & $1554.5(3)$ & $3191.7(8)$ & $6105(2)$ \\
\hline$Z$ & 2 & 4 & 8 \\
\hline$D_{\text {cale. }}\left(\mathrm{Mg} \cdot \mathrm{m}^{-3}\right)$ & 1.321 & 1.287 & 1.150 \\
\hline Radiation type & Mo $K \alpha$ & Mo $K \alpha$ & $\operatorname{MoK} \alpha$ \\
\hline$\mu\left(\mathrm{mm}^{-1}\right)$ & 0.094 & 0.092 & 0.077 \\
\hline Crystal size (mm) & $0.21 \times 0.21 \times 0.20$ & $0.23 \times 0.21 \times 0.2$ & $0.20 \times 0.12 \times 0.13$ \\
\hline$R_{\text {int }}$ & 0.0427 & 0.0349 & 0.1742 \\
\hline$\theta_{\max }\left[{ }^{\circ}\right]$ & 50.768 & 50.94 & 55.744 \\
\hline$\theta_{\min }\left[{ }^{\circ}\right]$ & 3.706 & 3.44 & 5.86 \\
\hline $\mathrm{R}_{1}[\mathrm{I} \geqslant \sigma(\mathrm{I})]$ & 0.0657 & 0.0467 & 0.1205 \\
\hline$w R_{2}$ & 0.1828 & 0.1354 & 0.2242 \\
\hline $\begin{array}{l}\text { No. of measured, } \\
\text { independent and } \\
\text { observed }[I>2 \sigma(I)] \\
\text { reflections }\end{array}$ & $15419,2859,2008$ & $30949,5890,3962$ & $93394,7016,4716$ \\
\hline $\begin{array}{c}\text { Largest diff. } \\
\text { peak/hole }\left(\mathrm{eA}^{-3}\right)\end{array}$ & $1.23,-0.68$ & $0.19,-0.16$ & $0.38,-0.24$ \\
\hline Parameter & 221 & 421 & 362 \\
\hline
\end{tabular}




\begin{tabular}{|c|c|c|c|}
\hline Crystal data & $2 b$ & 3d & 8 \\
\hline Chemical formula & $\mathrm{C}_{30} \mathrm{H}_{16} \mathrm{~B}_{2} \mathrm{Cl}_{4} \mathrm{~F}_{4} \mathrm{~N}_{4}$ & $\mathrm{C}_{32} \mathrm{H}_{24} \mathrm{~B}_{2} \mathrm{~F}_{4} \mathrm{~N}_{4} \mathrm{O}_{2}$ & $\mathrm{C}_{32} \mathrm{H}_{27} \mathrm{BF}_{2} \mathrm{~N}_{2}$ \\
\hline$M_{\mathrm{r}}$ & 671.89 & 594.17 & 488.36 \\
\hline $\begin{array}{c}\text { Crystal system, space } \\
\text { group }\end{array}$ & triclinic, $P-1$ & trigonal, $R 3 \mathrm{c}$ & triclinic, $P-1$ \\
\hline Temperature (K) & 293.15 & 273.15 & 293.15 \\
\hline$a, b, c(\AA)$ & $\begin{array}{l}14.1324(16), \\
14.8863(17), \\
15.9600(18)\end{array}$ & $\begin{array}{c}14.1306(9), \\
14.1306(9), \\
73.267(4)\end{array}$ & $\begin{array}{l}10.308(2), \\
12.549(3), \\
12.948(3)\end{array}$ \\
\hline$\alpha, \beta, \gamma\left(^{\circ}\right)$ & $\begin{array}{c}109.70(2), 106.536(2) \\
90.68(2)\end{array}$ & $\begin{array}{c}90.00 \\
90.00,120.00\end{array}$ & $\begin{array}{c}65.10,68.91(2) \\
71.62(2)\end{array}$ \\
\hline$V\left(\AA^{3}\right)$ & $3008.5(6)$ & $12669.5(14)$ & $1390.7(5)$ \\
\hline$Z$ & 4 & 18 & 2 \\
\hline$D_{\text {cale. }}\left(\mathrm{Mg} \cdot \mathrm{m}^{-3}\right)$ & 1.483 & 1.402 & 1.166 \\
\hline Radiation type & $\operatorname{MoK} \alpha(\lambda=0.71073)$ & $\operatorname{MoK} \alpha(\lambda=0.71073)$ & Mo $K \alpha(\lambda=0.71073)$ \\
\hline$\mu\left(\mathrm{mm}^{-1}\right)$ & 0.447 & 0.106 & 0.077 \\
\hline Crystal size (mm) & $0.23 \times 0.21 \times 0.20$ & $0.23 \times 0.20 \times 0.19$ & $0.23 \times 0.22 \times 0.21$ \\
\hline$R_{\text {int }}$ & 0.0551 & 0.0755 & 0.1536 \\
\hline$\theta_{\max }\left[^{\circ}\right]$ & 50.8 & 50.76 & 55.142 \\
\hline$\theta_{\min }\left[{ }^{\circ}\right]$ & 2.84 & 5.76 & 3.596 \\
\hline $\mathrm{R}_{1}[\mathrm{I} \geqslant \sigma(\mathrm{I})]$ & 0.0892 & 0.0359 & 0.0737 \\
\hline $\mathrm{wR}_{2}$ & 0.1692 & 0.0803 & 0.228 \\
\hline $\begin{array}{l}\text { No. of measured, } \\
\text { independent and } \\
\text { observed }[I>2 \sigma(I)] \\
\text { reflections }\end{array}$ & $29112,10658,5741$ & $142677,5172,4551$ & $12189,6281,4183$ \\
\hline $\begin{array}{l}\text { Largest diff. peak/hole } \\
\qquad\left(\mathrm{eA}^{-3}\right)\end{array}$ & $0.55,-0.27$ & $0.15,-0.18$ & $0.78,-0.27$ \\
\hline Parameter & 781 & 399 & 337 \\
\hline
\end{tabular}




\section{Photophysical date}

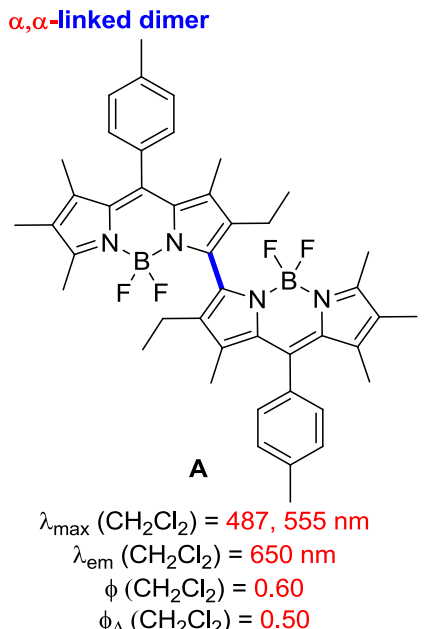

meso, $\beta$-linked dimers

meso,meso-linked dimer
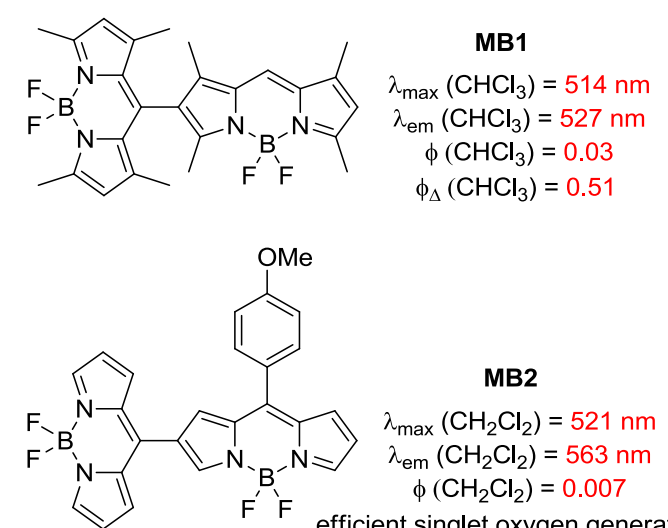

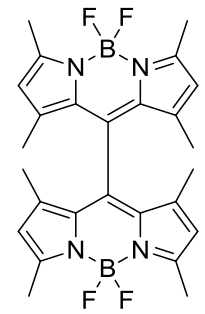

M

$\lambda_{\max }\left(\mathrm{CHCl}_{3}\right)=515 \mathrm{~nm}$ $\lambda_{\text {em }}\left(\mathrm{CHCl}_{3}\right)=588 \mathrm{~nm}$ $\phi\left(\mathrm{CHCl}_{3}\right)=0.31$ $\phi_{\Delta}\left(\mathrm{CHCl}_{3}\right)=0.46$

\section{$\beta, \beta$-linked dimers}

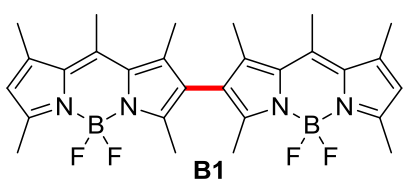

$\lambda_{\text {max }}\left(\mathrm{CH}_{2} \mathrm{Cl}_{2}\right)=526 \mathrm{~nm}$ $\lambda_{\text {em }}\left(\mathrm{CH}_{2} \mathrm{Cl}_{2}\right)=562 \mathrm{~nm}$ $\phi\left(\mathrm{CH}_{2} \mathrm{Cl}_{2}\right)=0.67$

fused ring-linked dimers<smiles></smiles>

$\lambda_{\text {max }}\left(\mathrm{CHCl}_{3}\right)=692 \mathrm{~nm}$

$\lambda_{\text {em }}\left(\mathrm{CHCl}_{3}\right)=703 \mathrm{~nm}$ $\phi\left(\mathrm{CHCl}_{3}\right)=0.72$

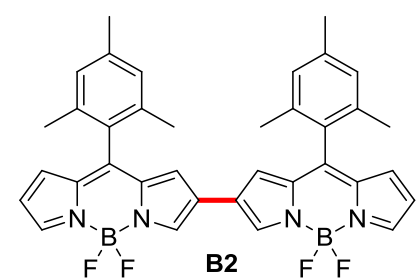

$\lambda_{\max }\left(\mathrm{CH}_{2} \mathrm{Cl}_{2}\right)=609 \mathrm{~nm}$ $\lambda_{\text {em }}\left(\mathrm{CH}_{2} \mathrm{Cl}_{2}\right)=655 \mathrm{~nm}$ $\phi\left(\mathrm{CH}_{2} \mathrm{Cl}_{2}\right)=0.15$

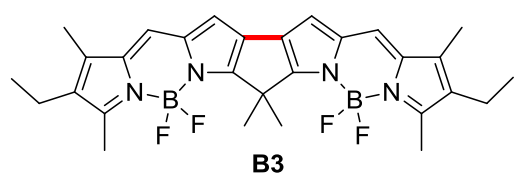

$\lambda_{\text {max }}\left(\mathrm{CH}_{2} \mathrm{Cl}_{2}\right)=688 \mathrm{~nm}$ $\lambda_{\text {em }}\left(\mathrm{CH}_{2} \mathrm{Cl}_{2}\right)=701 \mathrm{~nm}$ $\phi\left(\mathrm{CH}_{2} \mathrm{Cl}_{2}\right)=0.54$

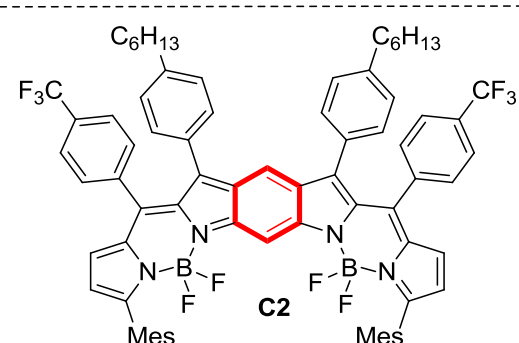

Mes $\lambda_{\text {max }}(\mathrm{THF})=629 \mathrm{~nm}$ $\lambda_{\text {em }}($ THF $)=940 \mathrm{~nm}$ $\phi(\mathrm{THF})<0.01$

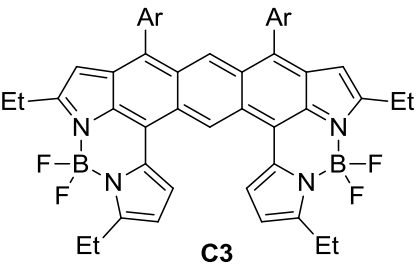

$\lambda_{\max }\left(\mathrm{CHCl}_{3}\right)=1136 \mathrm{~nm}$

$\phi:$ fluorescent quantum yield $\phi_{\Delta}:$ singlet oxygen luminescence yield

Chart S1. Previously reported representative BODIPY dimers $\left(\mathrm{A},{ }^{14} \mathrm{M},{ }^{15} \mathrm{MB} 1,{ }^{15}\right.$ $\mathrm{MB} 2,{ }^{16} \mathrm{~B} 1,{ }^{17} \mathrm{~B} 2,{ }^{18} \mathrm{B3},{ }^{19} \mathrm{C} 1,{ }^{20} \mathrm{C} 2,{ }^{21}$ and $\mathrm{C}^{22}$ ) and their photophysical properties: 
Table S4: Photophysical properties of dyes 2-9 in different solvents at room temperature (Data of starting BODIPYs 1 in dichloromethane were included for comparision).

\begin{tabular}{|c|c|c|c|c|c|c|}
\hline dyes & solvents & $\begin{array}{c}\lambda_{\text {abs }}^{\max } \\
(\mathrm{nm})\end{array}$ & $\begin{array}{c}\lambda_{\mathrm{em}}^{\max } \\
(\mathrm{nm})\end{array}$ & $\varepsilon_{\mathrm{abs}\left(10^{-4}\right)^{\mathrm{a}}}$ & $\phi^{\mathrm{b}}$ & $\begin{array}{c}\text { Stokes Shift } \\
\left(\mathrm{cm}^{-1}\right)\end{array}$ \\
\hline $1 \mathbf{a}$ & dichloromethane & 500 & 512 & 4.9 & 0.92 & 470 \\
\hline \multirow{5}{*}{$2 \mathbf{a}$} & hexane & 668 & 703 & 7.2 & 0.71 & 760 \\
\hline & toluene & 673 & 708 & 7.5 & 0.65 & 740 \\
\hline & dichloromethane & 663 & 703 & 6.9 & 0.71 & 880 \\
\hline & acetonitrile & 651 & 692 & 7.4 & 0.72 & 910 \\
\hline & methanol & 653 & 692 & 7.2 & 0.60 & 860 \\
\hline \multirow[t]{5}{*}{$\mathbf{3 a}$} & hexane & 570 & 641 & 5.3 & 0.18 & 1970 \\
\hline & toluene & 578 & 664 & 5.1 & 0.14 & 2280 \\
\hline & dichloromethane & 575 & 678 & 4.6 & 0.06 & 2640 \\
\hline & acetonitrile & 563 & 689 & 4.5 & 0.01 & 3260 \\
\hline & methanol & 565 & 677 & 4.8 & 0.02 & 2900 \\
\hline $\mathbf{1 b}$ & dichloromethane & 512 & 525 & 5.0 & 0.75 & 480 \\
\hline \multirow[t]{5}{*}{$2 b$} & hexane & 693 & 725 & 7.5 & 0.41 & 640 \\
\hline & toluene & 698 & 732 & 7.2 & 0.40 & 660 \\
\hline & dichloromethane & 685 & 725 & 7.4 & 0.50 & 800 \\
\hline & acetonitrile & 667 & 709 & 6.5 & 0.56 & 890 \\
\hline & methanol & 677 & 712 & 8.2 & 0.37 & 730 \\
\hline \multirow[t]{5}{*}{$3 \mathbf{b}$} & hexane & 585 & 667 & 6.5 & $0.14^{\mathrm{c}}$ & 2100 \\
\hline & toluene & 593 & 678 & 6.6 & 0.22 & 2120 \\
\hline & dichloromethane & 587 & 692 & 5.3 & 0.12 & 2685 \\
\hline & acetonitrile & 574 & 700 & 5.8 & 0.05 & 3140 \\
\hline & methanol & 576 & 688 & 6.5 & 0.05 & 2830 \\
\hline 1c & dichloromethane & 500 & 520 & 4.7 & 0.06 & 770 \\
\hline \multirow[t]{5}{*}{$2 c$} & hexane & 664 & 703 & 6.5 & 0.70 & 835 \\
\hline & toluene & 668 & 708 & 6.1 & 0.69 & 860 \\
\hline & dichloromethane & 653 & 701 & 5.6 & 0.72 & 1050 \\
\hline & acetonitrile & 641 & 689 & 4.9 & 0.70 & 1090 \\
\hline & methanol & 646 & 690 & 6.2 & 0.65 & 990 \\
\hline \multirow[t]{5}{*}{$3 c$} & hexane & 554 & 654 & 5.9 & 0.07 & 2780 \\
\hline & toluene & 561 & 670 & 5.1 & 0.12 & 2900 \\
\hline & dichloromethane & 553 & 685 & 5.0 & 0.06 & 3485 \\
\hline & acetonitrile & 542 & 695 & 5.0 & 0.003 & 4060 \\
\hline & methanol & 544 & 680 & 4.8 & 0.006 & 3680 \\
\hline
\end{tabular}




\begin{tabular}{|c|c|c|c|c|c|c|}
\hline 1d & dichloromethane & 496 & 514 & 4.5 & 0.08 & 710 \\
\hline \multirow[t]{5}{*}{ 2d } & hexane & 663 & 700 & 6.5 & 0.60 & 810 \\
\hline & toluene & 666 & 706 & 6.0 & 0.67 & 860 \\
\hline & dichloromethane & 651 & 697 & 5.4 & 0.67 & 1020 \\
\hline & acetonitrile & 639 & 689 & 5.7 & 0.72 & 1160 \\
\hline & methanol & 642 & 689 & 5.8 & 0.61 & 1060 \\
\hline \multirow[t]{5}{*}{ 3d } & hexane & 553 & 652 & 5.6 & 0.11 & 2760 \\
\hline & toluene & 561 & 672 & 5.2 & 0.09 & 2940 \\
\hline & dichloromethane & 552 & 687 & 4.5 & 0.05 & 3560 \\
\hline & acetonitrile & 542 & 698 & 4.4 & 0.003 & 4140 \\
\hline & methanol & 545 & 680 & 4.7 & 0.004 & 3640 \\
\hline 1e & dichloromethane & 518 & 535 & 4.5 & 0.56 & 610 \\
\hline \multirow[t]{5}{*}{$2 e$} & hexane & 700 & 735 & 6.8 & 0.27 & 680 \\
\hline & toluene & 708 & 751 & 6.3 & 0.28 & 820 \\
\hline & dichloromethane & 692 & 735 & 5.6 & 0.29 & 840 \\
\hline & acetonitrile & 694 & 719 & 6.1 & 0.30 & 570 \\
\hline & methanol & 680 & 723 & 6.3 & 0.21 & 880 \\
\hline \multirow[t]{5}{*}{$3 \mathbf{e}$} & hexane & 548 & 691 & 5.6 & 0.10 & 3770 \\
\hline & toluene & 556 & 703 & 5.3 & 0.09 & 3750 \\
\hline & dichloromethane & 550 & 705 & 4.9 & 0.05 & 4000 \\
\hline & acetonitrile & 544 & 733 & 5.0 & 0.01 & 4740 \\
\hline & methanol & 544 & 731 & 4.4 & 0.02 & 4700 \\
\hline \multirow[t]{5}{*}{5} & hexane & 708 & 766 & 7.6 & 0.23 & 1070 \\
\hline & toluene & 712 & 775 & 7.0 & 0.24 & 1140 \\
\hline & dichloromethane & 702 & 774 & 6.3 & 0.15 & 1320 \\
\hline & acetonitrile & 686 & 759 & 6.4 & 0.03 & 1400 \\
\hline & methanol & 694 & 760 & 6.8 & 0.06 & 1250 \\
\hline \multirow[t]{5}{*}{6} & hexane & 746 & 806 & 8.4 & 0.13 & 1000 \\
\hline & toluene & 750 & 817 & 7.5 & 0.13 & 1090 \\
\hline & dichloromethane & 742 & 813 & 6.5 & 0.11 & 1180 \\
\hline & acetonitrile & 722 & 796 & 7.1 & 0.09 & 1290 \\
\hline & methanol & 734 & 798 & 7.3 & 0.08 & 1090 \\
\hline \multirow[t]{5}{*}{7} & hexane & 760 & 795 & 3.6 & 0.01 & 580 \\
\hline & toluene & 760 & 808 & 3.3 & 0.01 & 690 \\
\hline & dichloromethane & 710 & 802 & 3.3 & 0.004 & 1440 \\
\hline & acetonitrile & 702 & 783 & 3.1 & 0.002 & 1470 \\
\hline & methanol & 700 & 781 & 3.3 & 0.003 & 1500 \\
\hline
\end{tabular}




\begin{tabular}{|c|c|c|c|c|c|c|}
\hline $\mathbf{8}$ & hexane & 570 & 585 & 5.3 & 0.65 & 470 \\
& toluene & 574 & 589 & 5.1 & 0.53 & 440 \\
& dichloromethane & 570 & 590 & 4.8 & 0.25 & 590 \\
& acetonitrile & 564 & 584 & 4.8 & 0.17 & 610 \\
& methanol & 565 & 586 & 5.3 & 0.17 & 630 \\
\hline 9a & hexane & 508 & 530 & 7.4 & 0.87 & 820 \\
& toluene & 510 & 533 & 8.2 & 0.85 & 850 \\
& dichloromethane & 508 & 530 & 8.3 & 0.84 & 820 \\
& acetonitrile & 506 & 527 & 8.2 & 0.81 & 790 \\
& methanol & 506 & 527 & 6.3 & 0.82 & 790 \\
\hline 9b & hexane & 520 & 522 & 6.2 & 0.85 & 80 \\
& toluene & 520 & 522 & 6.0 & 0.83 & 180 \\
& dichloromethane & 518 & 523 & 6.9 & 0.85 & 190 \\
& acetonitrile & 516 & 520 & 6.0 & 0.73 & 150 \\
& methanol & 516 & 519 & 6.8 & 0.80 & 110 \\
\hline
\end{tabular}

${ }^{a}$ date corresponding to the strongest absorption maximum, the unit for $\varepsilon$ is $\mathrm{M}^{-1} \mathrm{~cm}^{-1}$. The standard errors are less than $10 \%$ from three independent measurements. ${ }^{b}$ Fluorescence quantum yields of 1a, 1b, 1c, 1d and 1e were calculated using Fluorescein ( $\Phi=0.9$ in $0.1 \mathrm{M} \mathrm{NaOH}$ ) as the reference. Fluorescence quantum yields of 8, 9a and 9b were calculated using Rhodamine B $(\Phi=0.49$ in ethanol) as the reference. Fluorescence quantum yields of $\mathbf{2 a}, \mathbf{2 b}, \mathbf{2 c}, \mathbf{2 d}, \mathbf{2 e}, \mathbf{5}$ and $\mathbf{7}$ were calculated using 1,7diphenyl-3,5-di(4-methoxyphenyl) -azadipyrromethene ( $\Phi=0.36$ in chloroform) as the reference. Fluorescence quantum yields of $\mathbf{3 a}, \mathbf{3 b}, \mathbf{3 c}, \mathbf{3 d}$ and $\mathbf{3 e}$ were calculated using Cresyl violet perchlorate $(\Phi=0.54$ in methanol) as the reference. Fluorescence quantum yields of $\mathbf{6}$ was calculated using Indocyanine Green $(\Phi=0.12$ in dimethyl sulfoxide) as the reference. ${ }^{\mathrm{c}} 15 \mathrm{v} \%$ dichloromethane was added. 

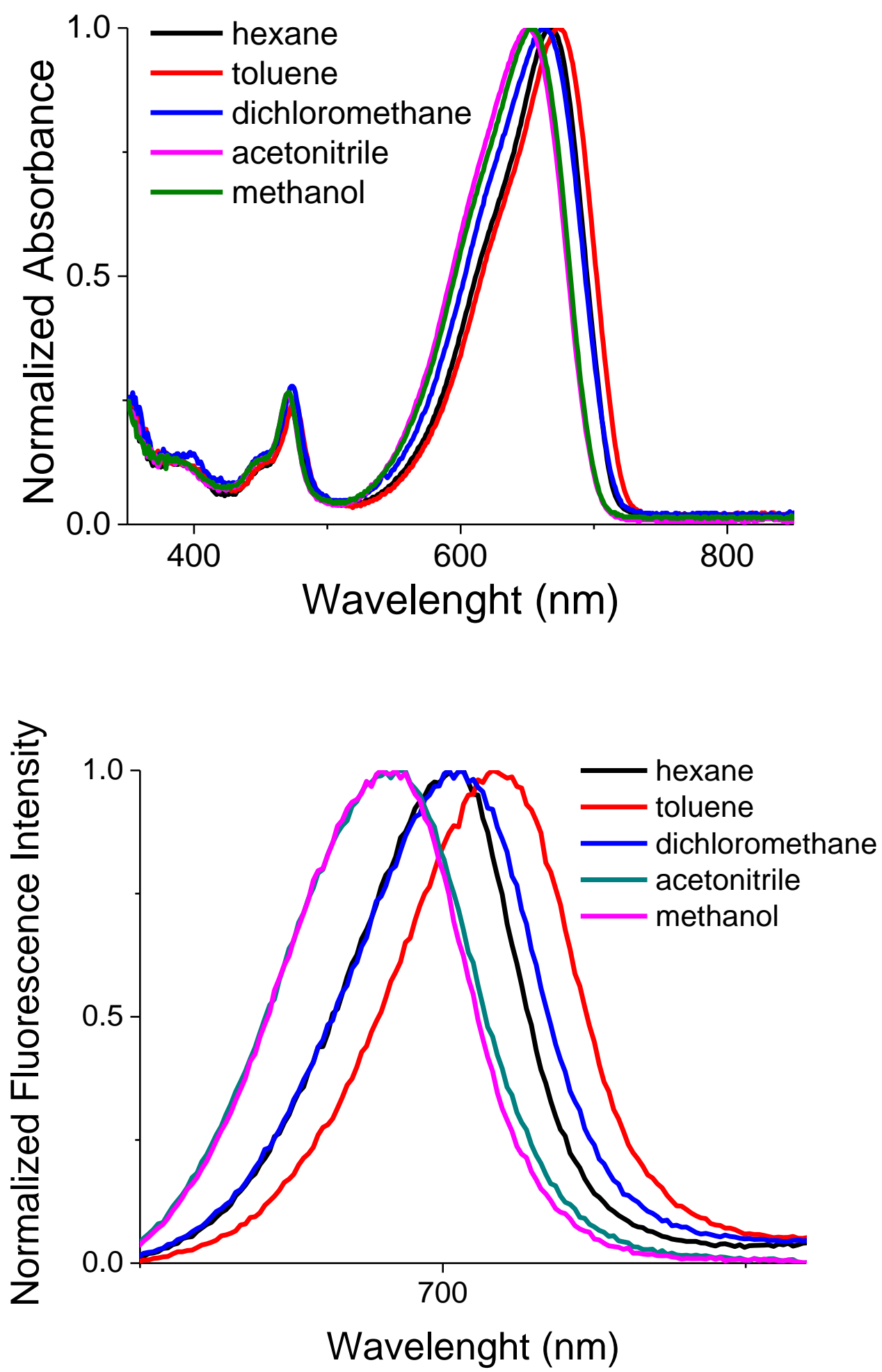

Figure S5: Absorption (top) and emission (bottom) spectra of compound 2a recorded in different solvents. Excited at $640 \mathrm{~nm}$. 

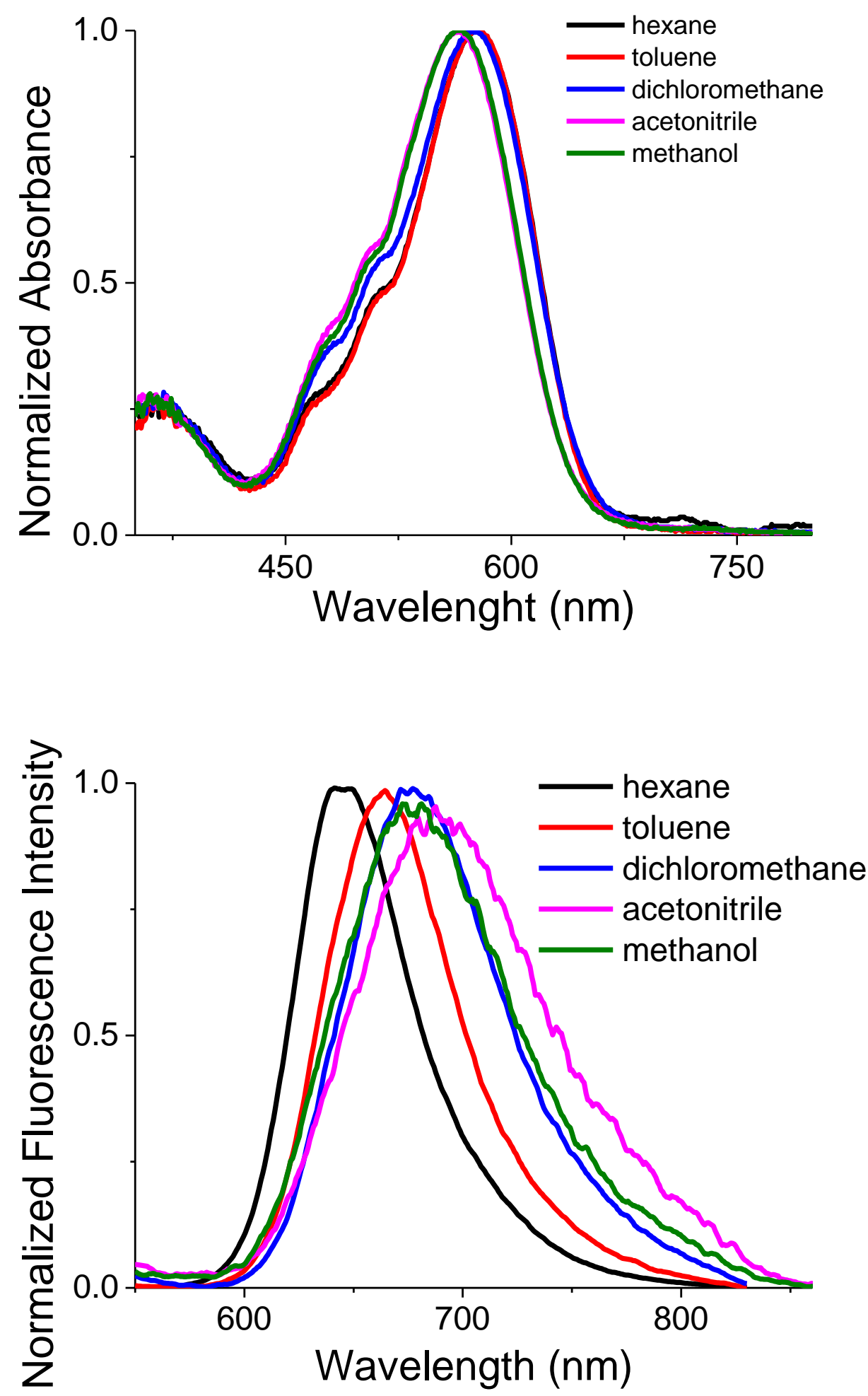

Figure S6: Absorption (top) and emission (bottom) spectra of compound 3a recorded in different solvents. Excited at $530 \mathrm{~nm}$. 

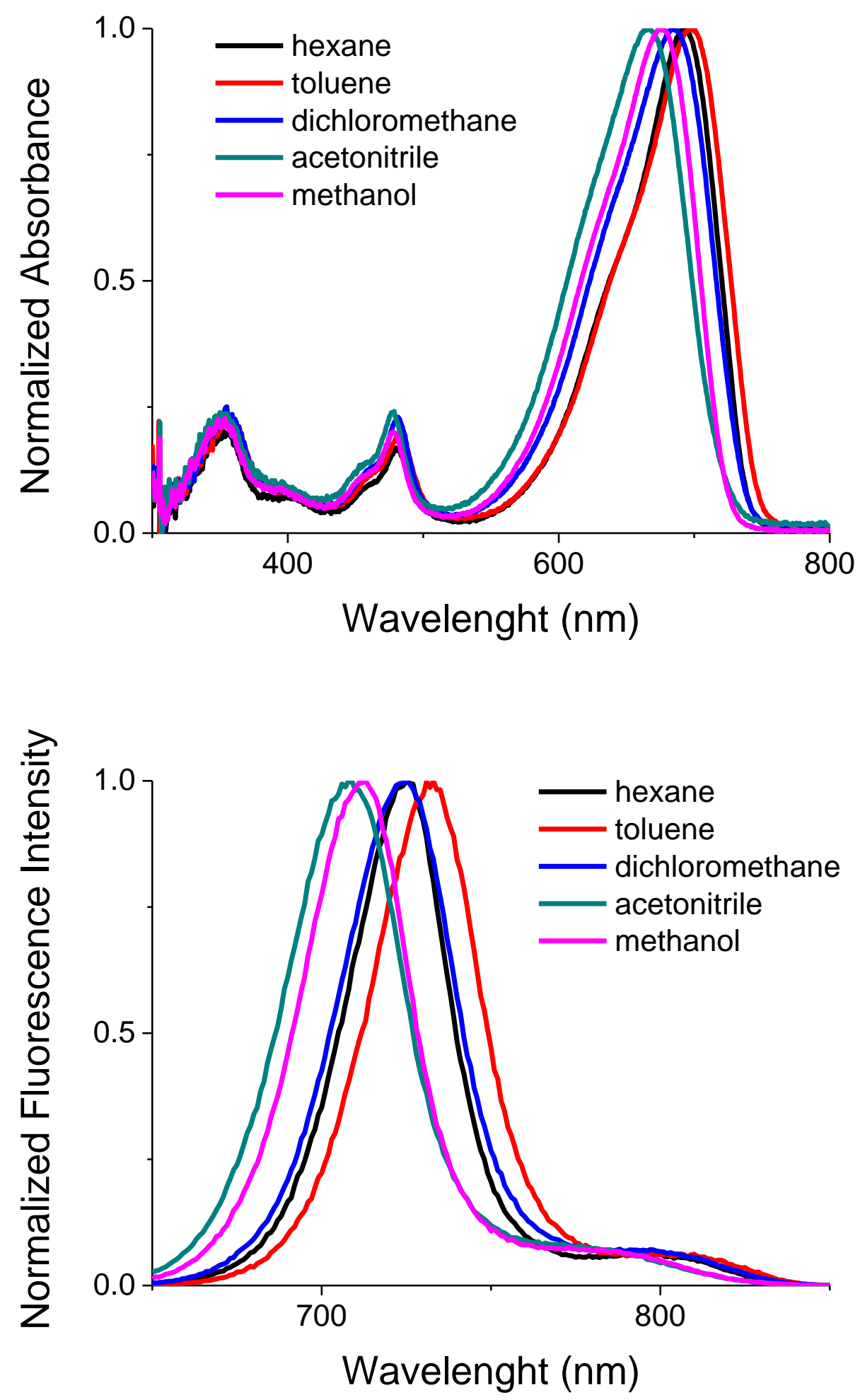

Figure S7: Absorption (top) and emission (bottom) spectra of compound 2b recorded in different solvents. Excited at $640 \mathrm{~nm}$ 

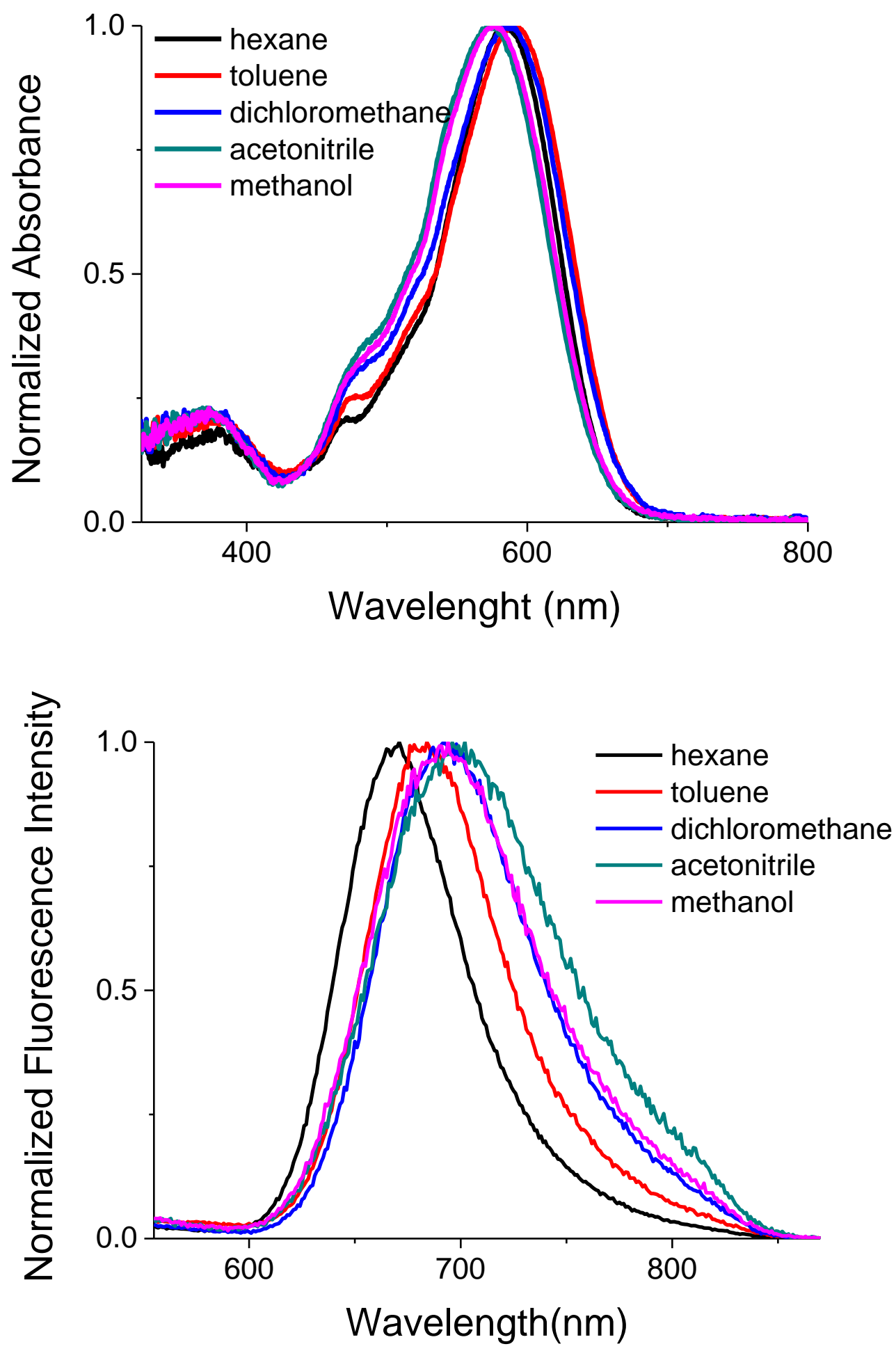

Figure S8: Absorption (top) and emission (bottom) spectra of compound $\mathbf{3 b}$ recorded in different solvents. Excited at $540 \mathrm{~nm}$. 

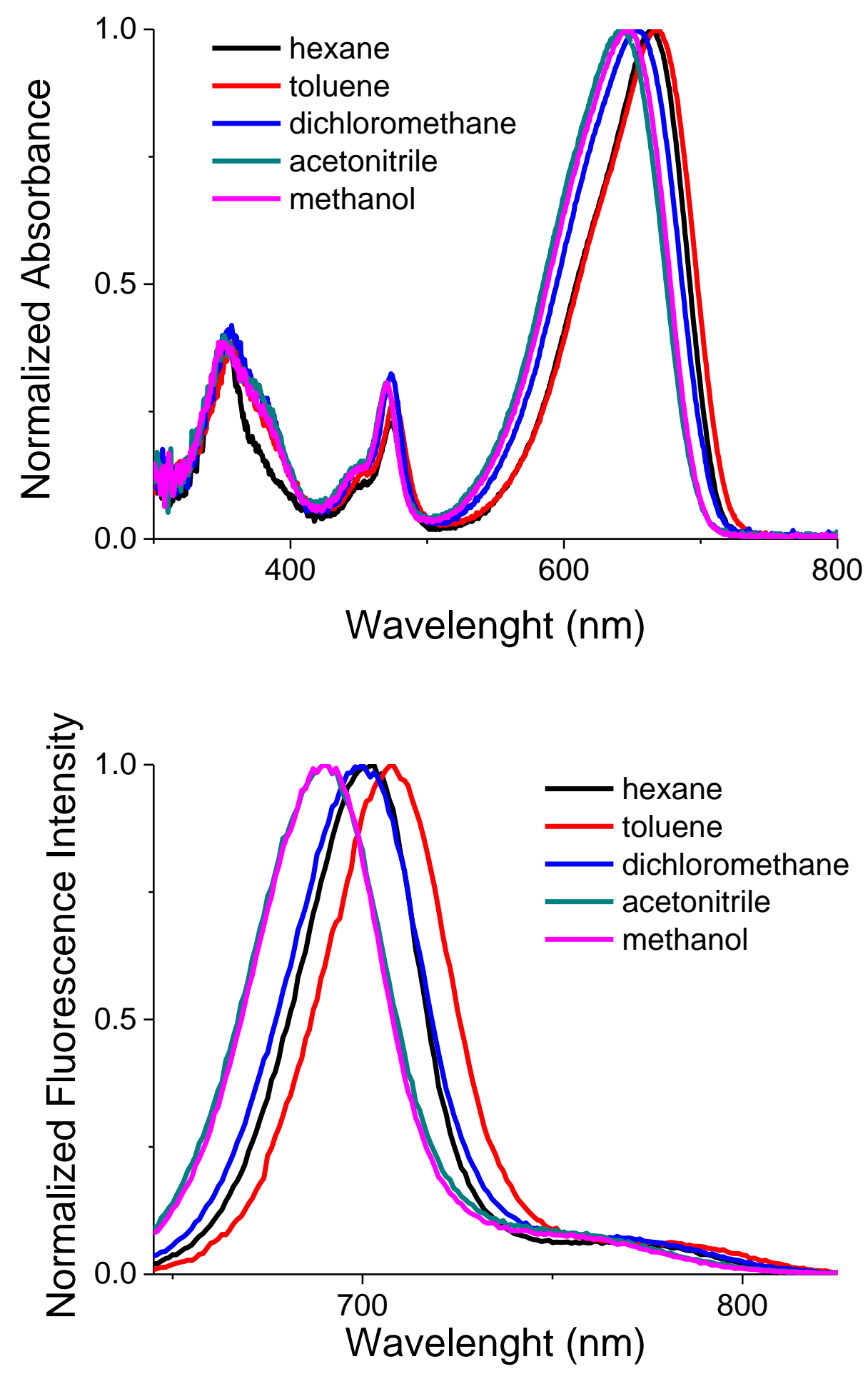

Figure S9: Absorption (top) and emission (bottom) spectra of compound 2c recorded in different solvents. Excited at $640 \mathrm{~nm}$. 

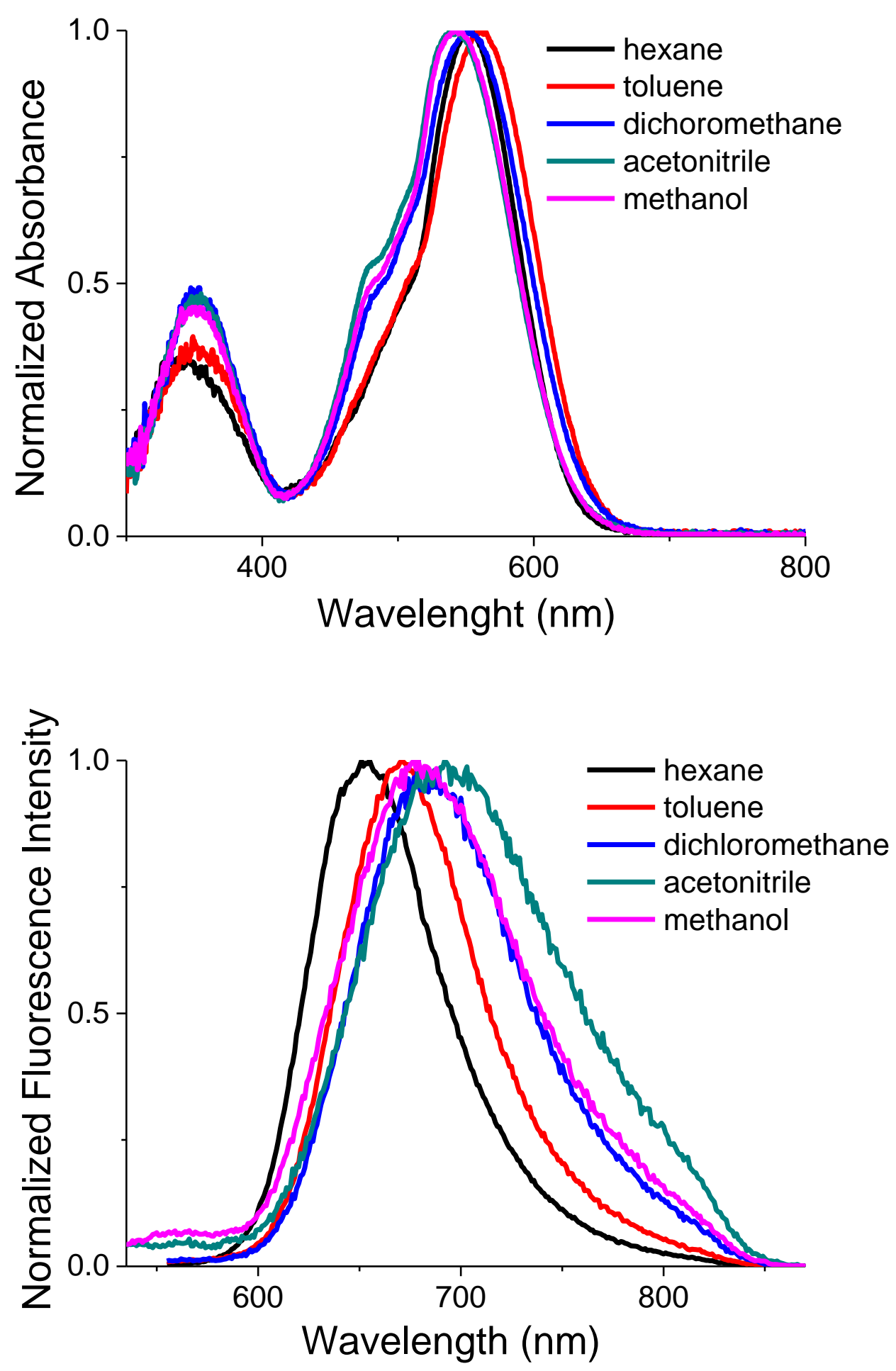

Figure S10: Absorption (top) and emission (bottom) spectra of compound 3c recorded in different solvents. Excited at $530 \mathrm{~nm}$. 

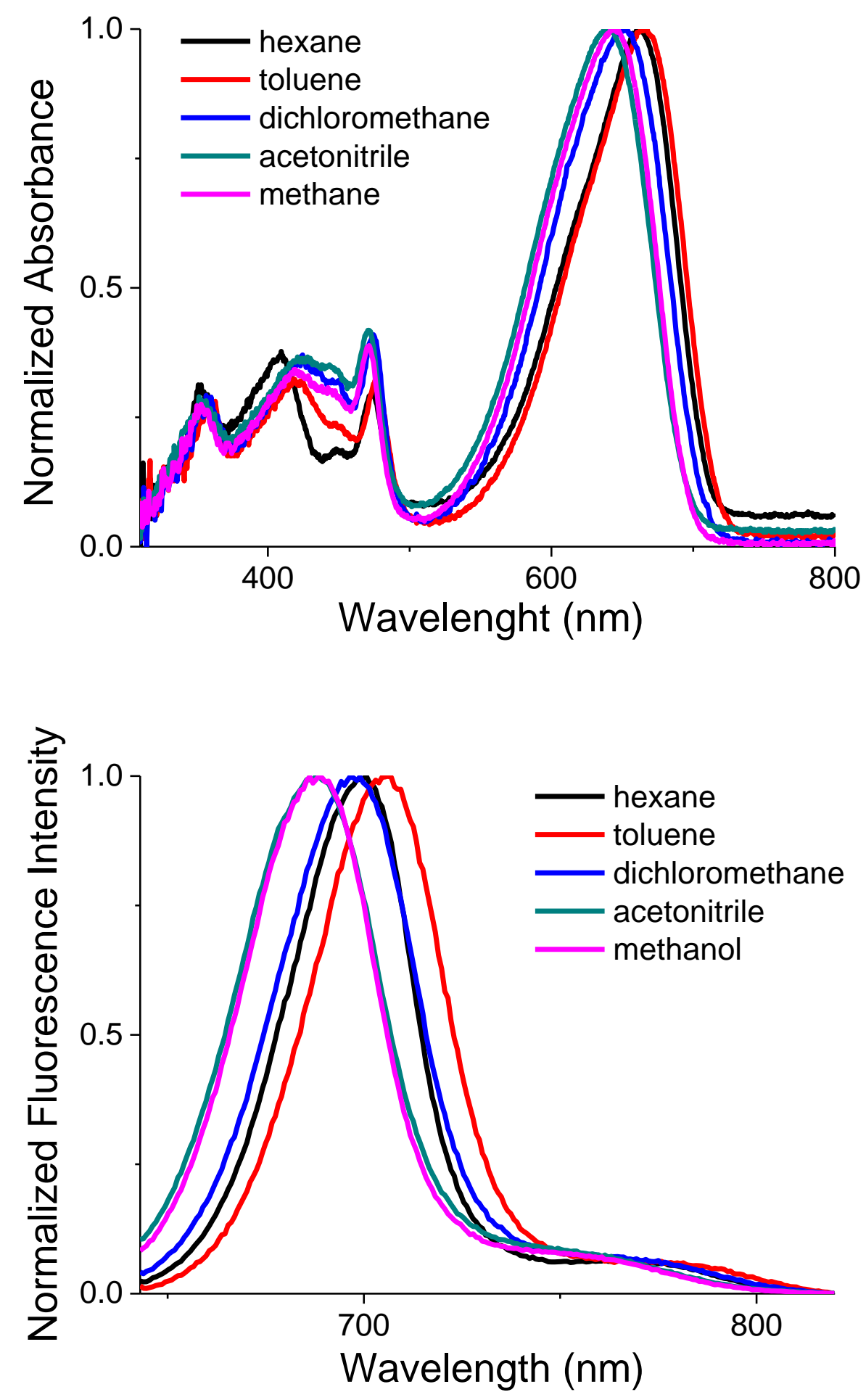

Figure S11: Absorption (top) and emission (bottom) spectra of compound 2d recorded in different solvents. Excited at $640 \mathrm{~nm}$. 

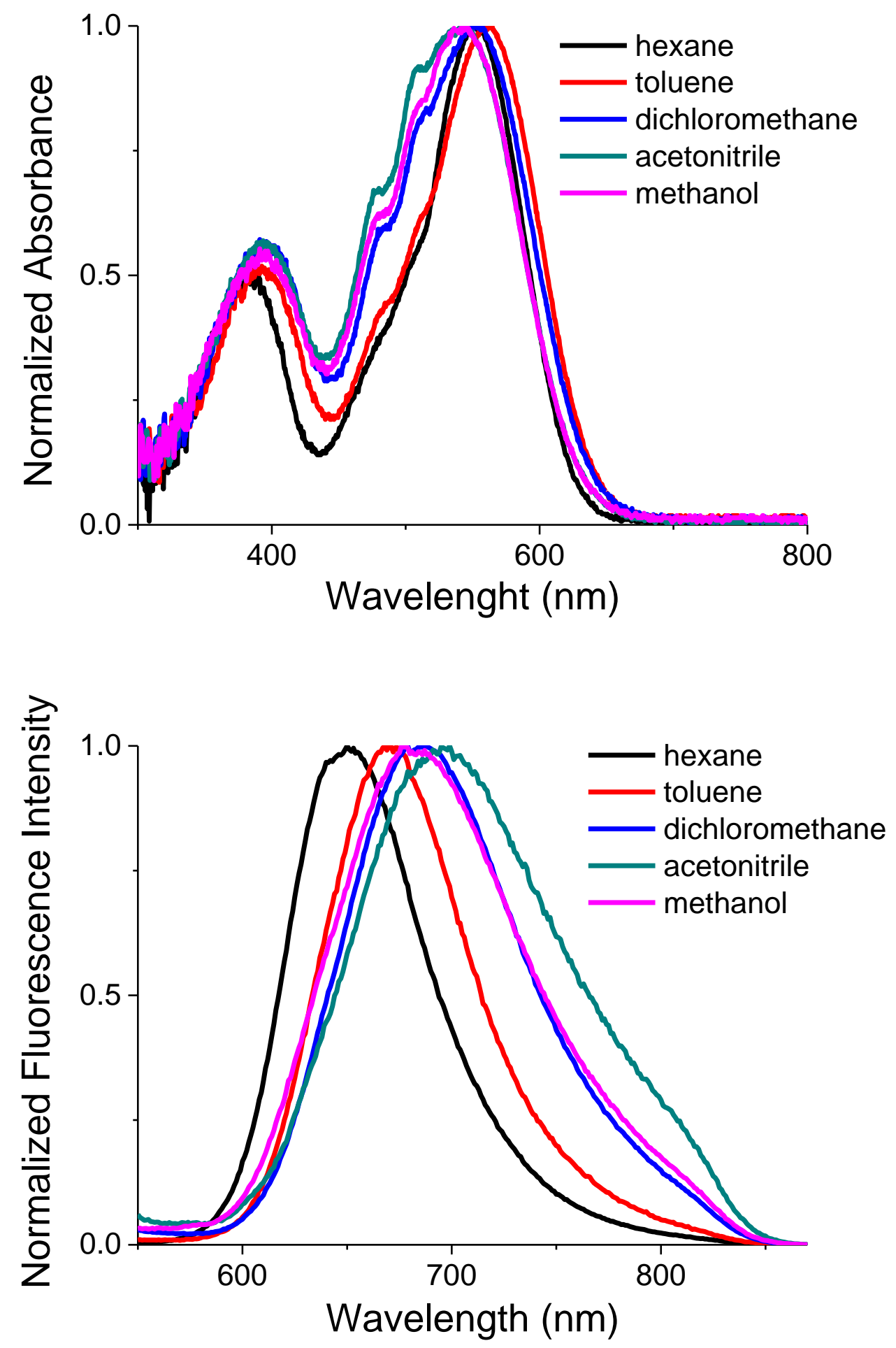

Figure S12: Absorption (top) and emission (bottom) spectra of compound 3d recorded in different solvents. Excited at $530 \mathrm{~nm}$. 

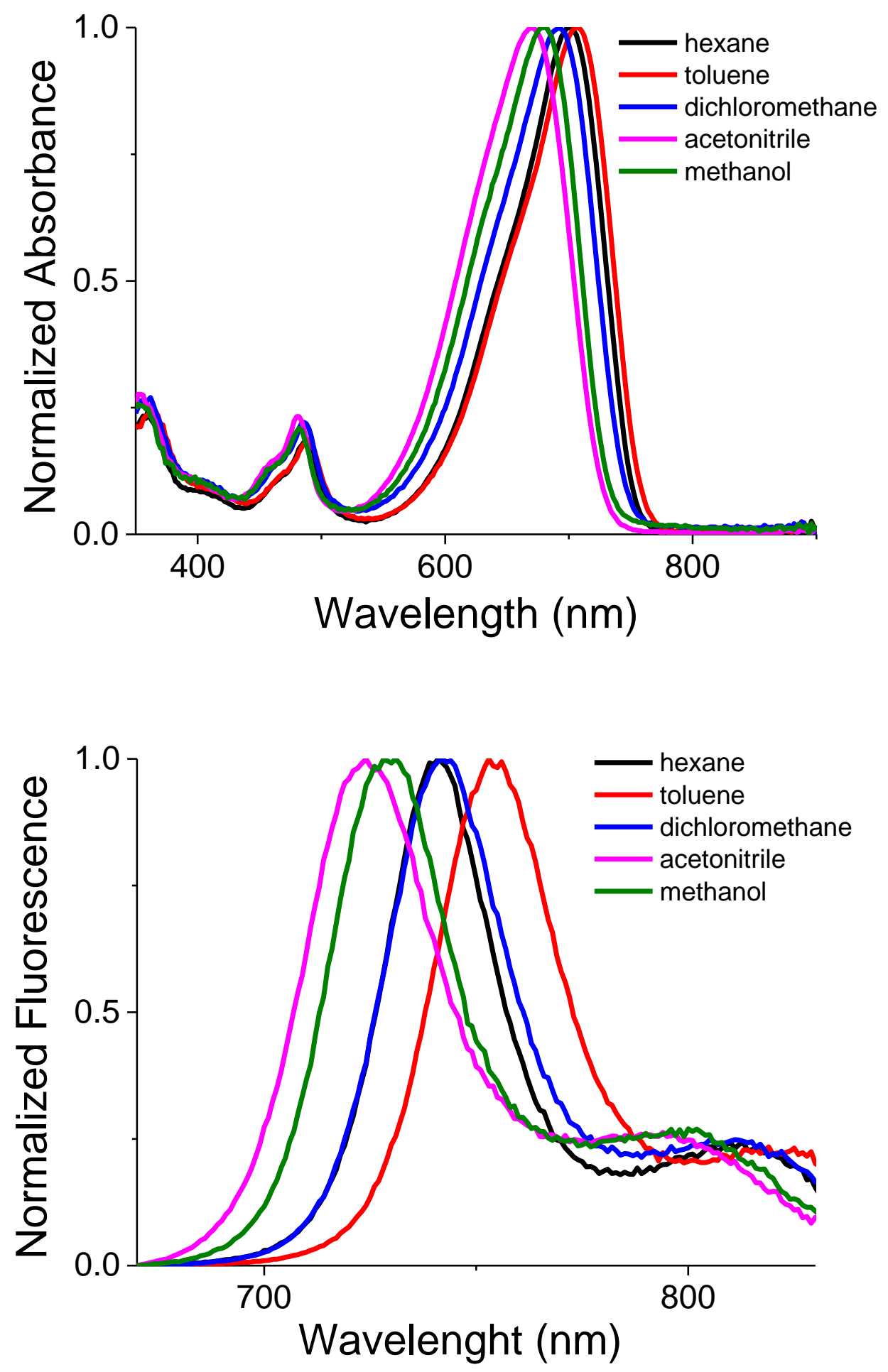

Figure S13: Absorption (top) and emission (bottom) spectra of compound 2e recorded in different solvents. Excited at $650 \mathrm{~nm}$. 

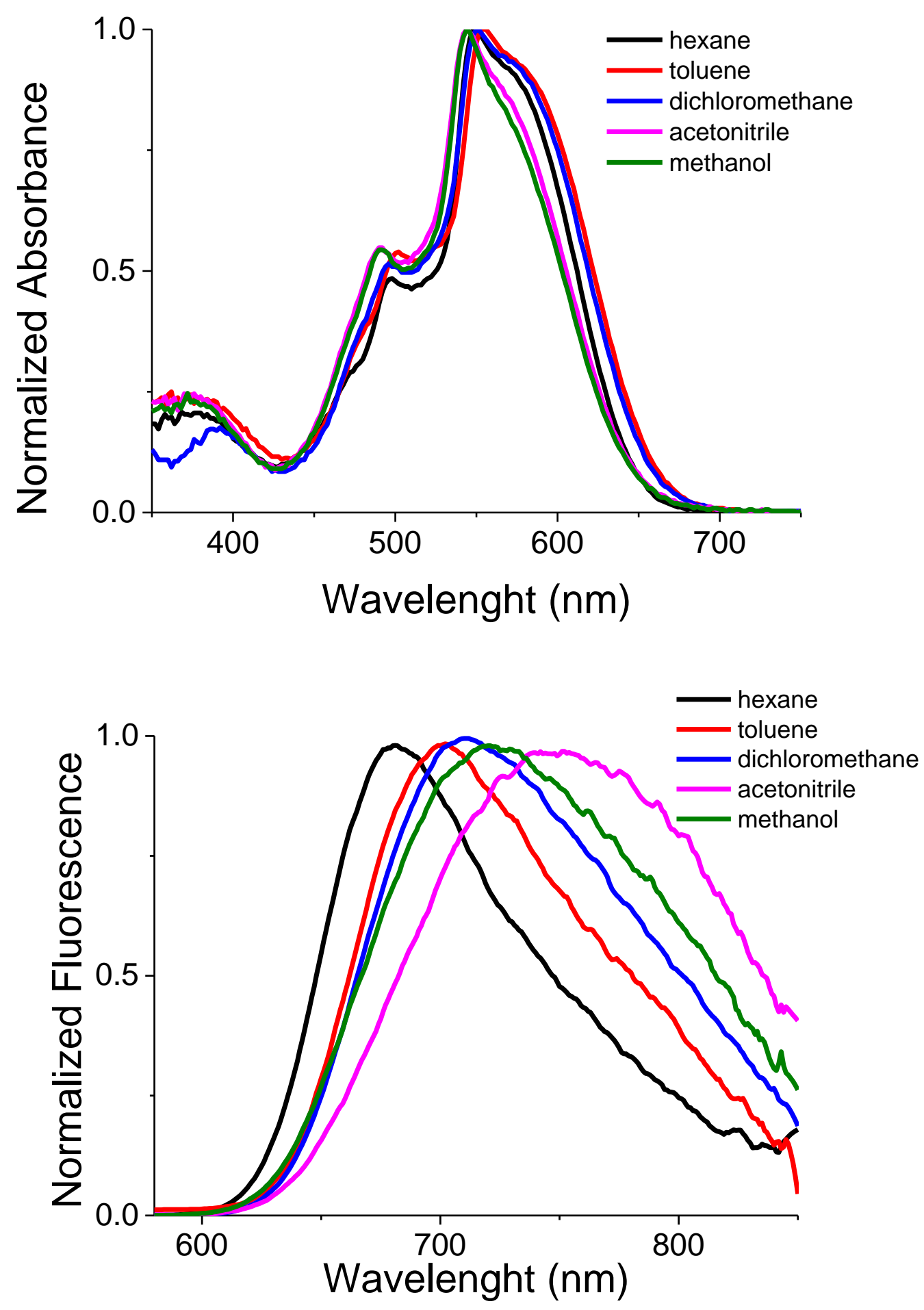

Figure S14: Absorption (top) and emission (bottom) spectra of compound 3e recorded in different solvents. Excited at $530 \mathrm{~nm}$ 

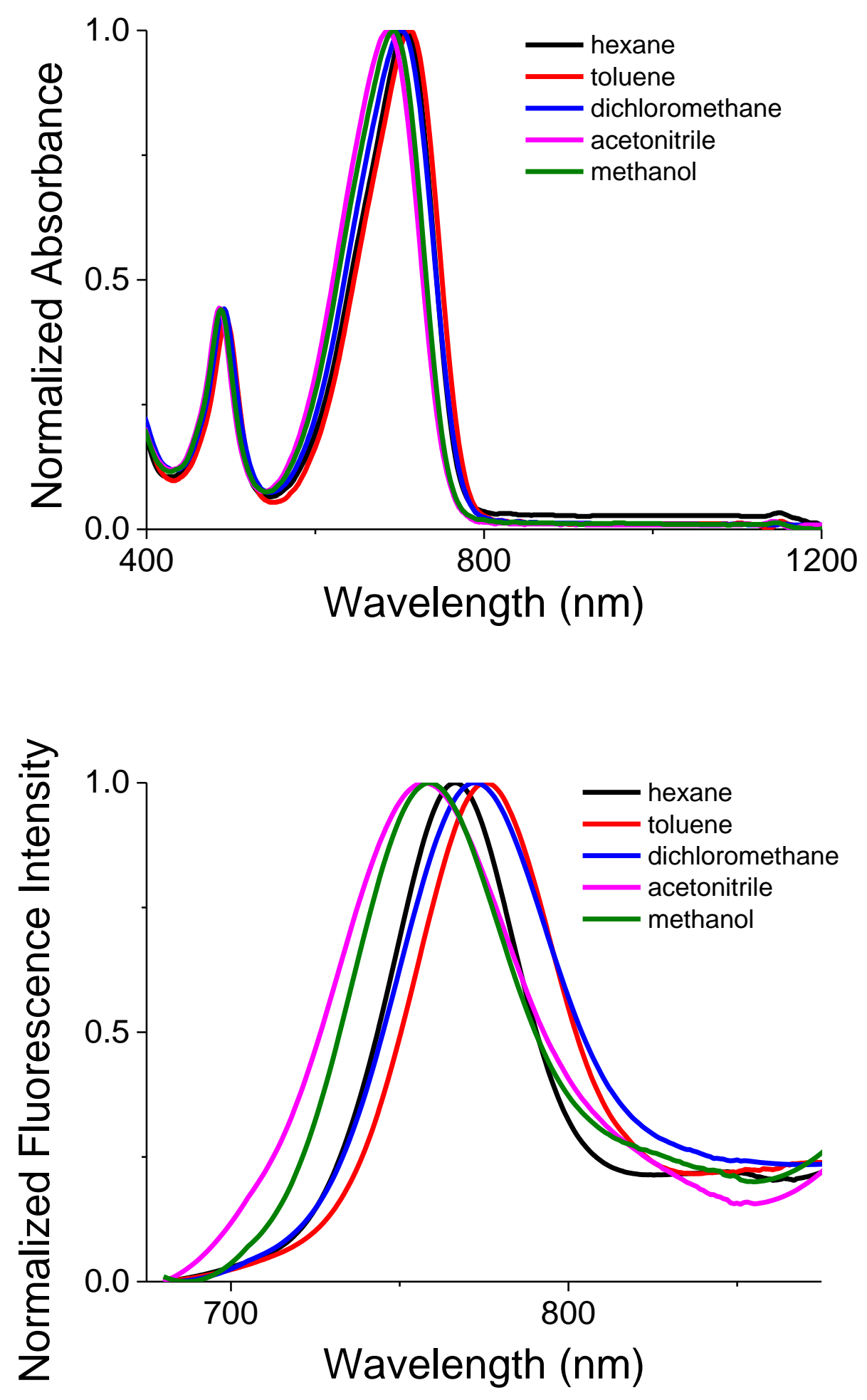

Figure S15. Normalized absorption (top) and Normalized fluorescence emission (bottom) spectra of 5 recorded in different solvents; excited at $670 \mathrm{~nm}$. 

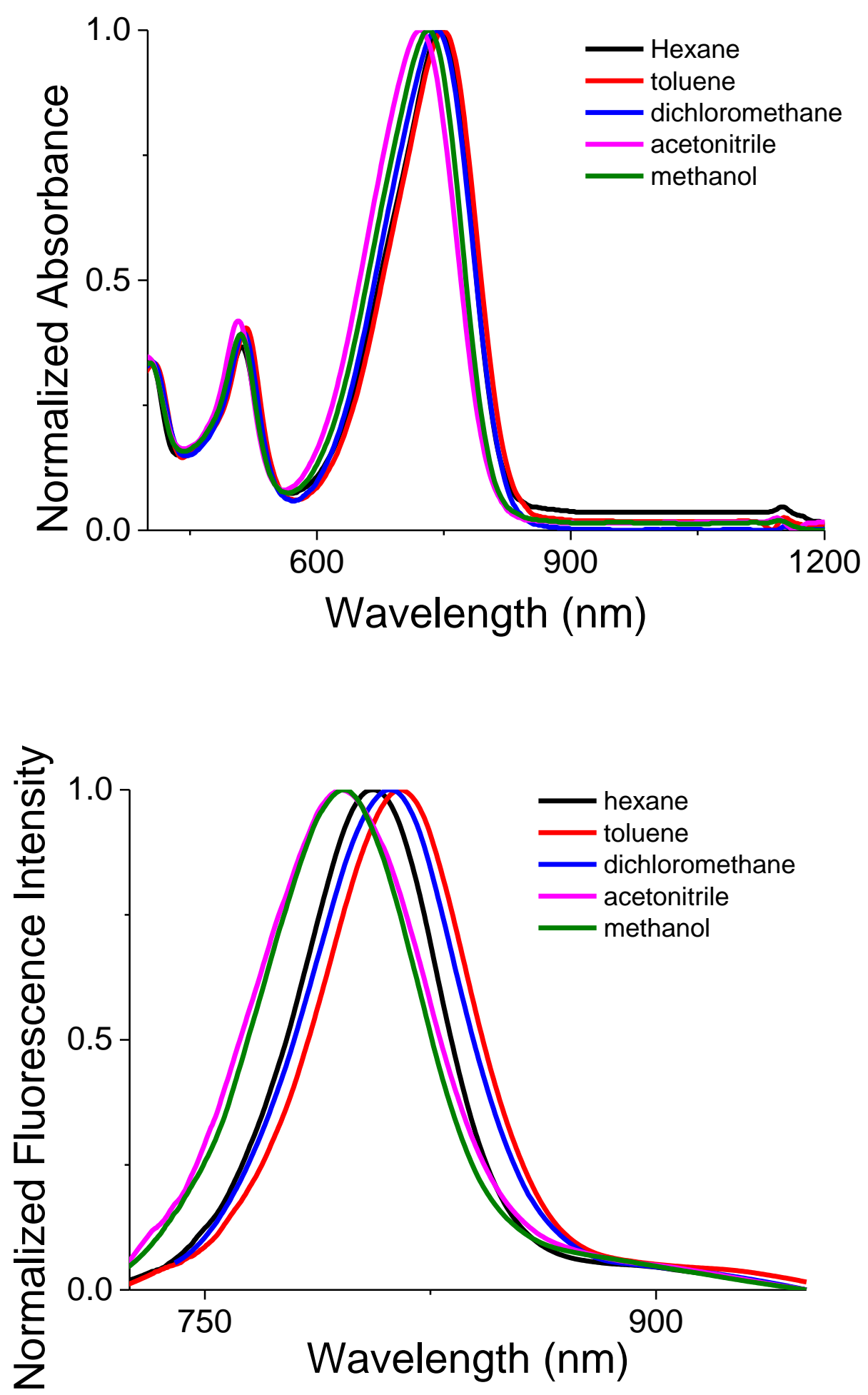

Figure S16. Normalized absorption (top) and Normalized fluorescence emission (bottom) spectra of 6 recorded in different solvents; excited at $730 \mathrm{~nm}$. 

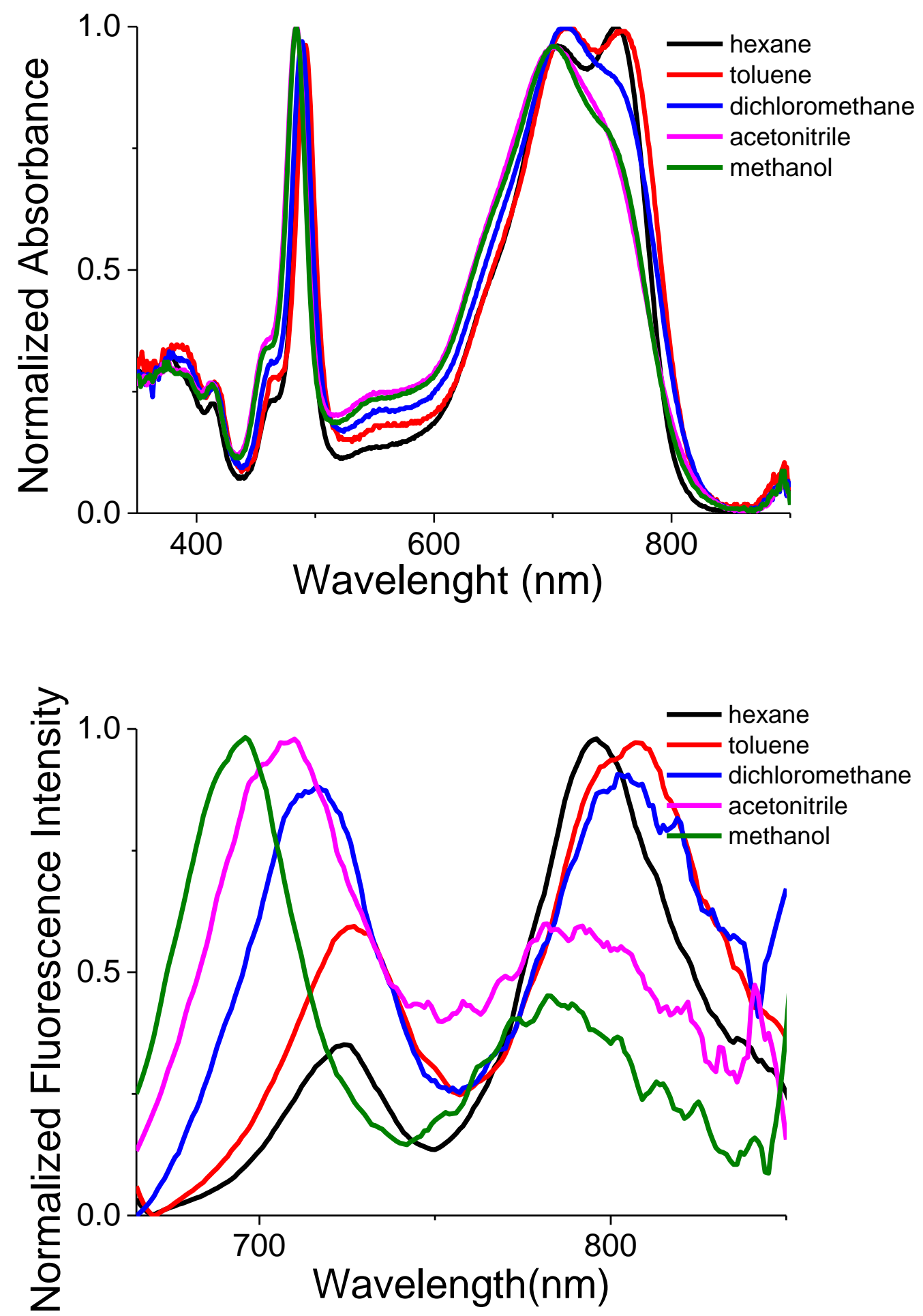

Figure S17: Absorption (top) and emission (bottom) spectra of compound 7 recorded in different solvents. Excited at $660 \mathrm{~nm}$. 

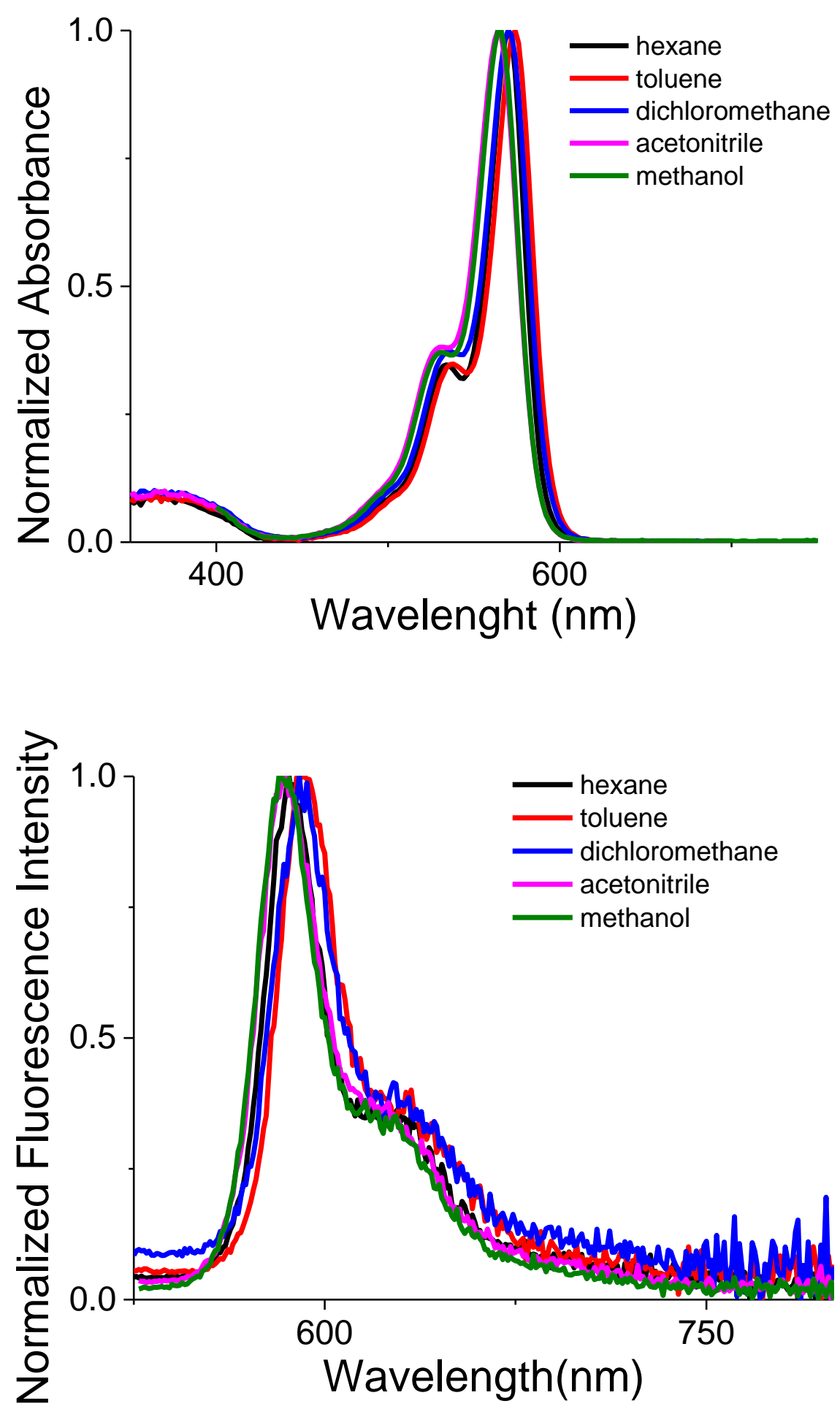

Figure S18: Absorption (top) and emission (bottom) spectra of compound 8 recorded in different solvents. Excited at $520 \mathrm{~nm}$ 

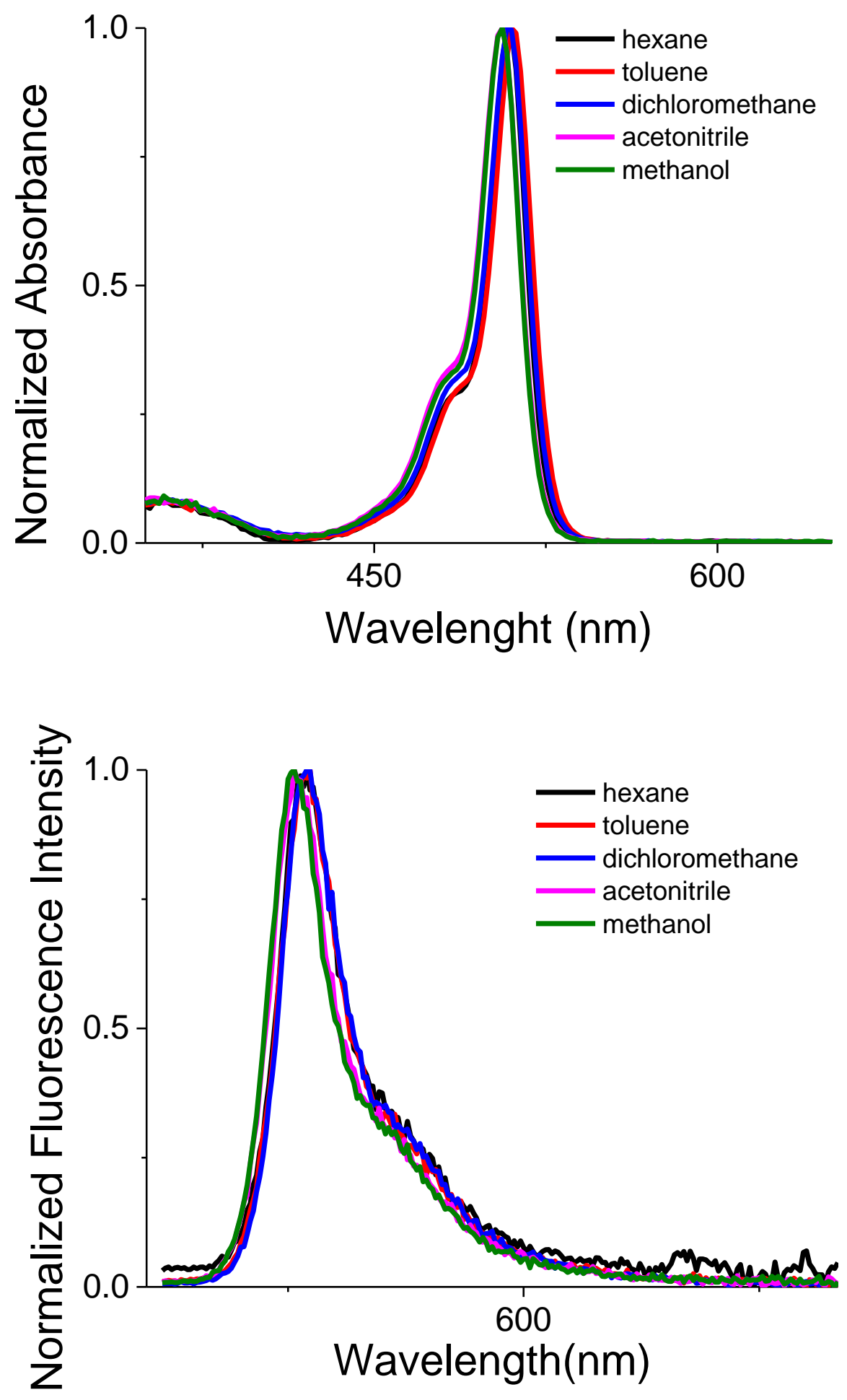

Figure S19: Absorption (top) and emission (bottom) spectra of compound 9a recorded in different solvents. Excited at $485 \mathrm{~nm}$ 

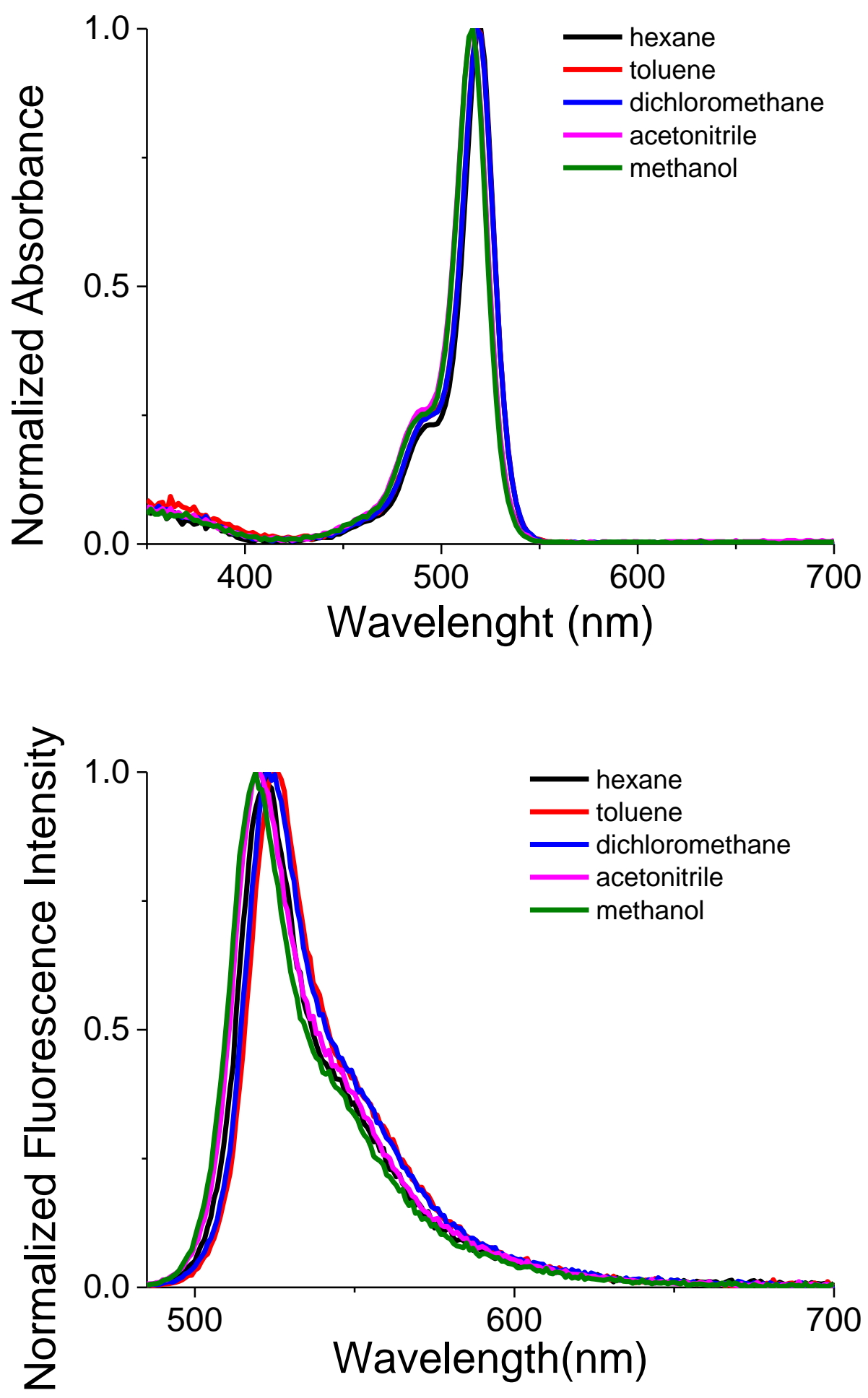

Figure S20: Absorption (top) and emission (bottom) spectra of compound 9b recorded in different solvents. Excited at $485 \mathrm{~nm}$ 


\section{Singlet oxygen generation properties}

A comparative study of the relative singlet oxygen generating efficiency of these dyes $\left(5 \times 10^{-6} \mathrm{M}\right)$ was performed in air-saturated solvents under light at $532 \mathrm{~nm}$ irradiation $\left(10 \mathrm{~mW} / \mathrm{cm}^{2}\right)$ condition using 1,3 diphenylisobenzofuran (DPBF, $\left.4 \times 10^{-5} \mathrm{M}\right)$ as a trap molecule. ${ }^{23}$ A wellknown photosensitizer against Rose Bengal (RB) sodium salt (1× $10^{-5} \mathrm{M}, \Phi_{\Delta}=0.8$ in methanol) was used as reference. The decrease of the absorbance band of DPBF at $410 \mathrm{~nm}$ was monitored. Singlet oxygen quantum yield $\left(\Phi_{\Delta}\right)$ determinations were carried out using the chemical trapping method. The $\Phi_{\Delta}$ value was obtained by the relative method using $\mathbf{R B}$ as the reference as shown in eqn (1)

$\Phi_{\Delta \mathrm{sam}}=\Phi_{\Delta \mathrm{ref}}\left[\left(\mathrm{m}_{\mathrm{sam}} / \mathrm{m}_{\mathrm{ref}}\right)\left(\mathrm{L}_{\mathrm{ref}} / \mathrm{L}_{\mathrm{sam}}\right)\right]$

Where $\Phi_{\Delta \text { ref }}$ and $\Phi_{\Delta \text { sam }}$ are the singlet oxygen quantum yield for the standard $\mathbf{R B}$ and photosensitizer (3a, 3b, 3c, $\mathbf{3 d}, \mathbf{3 e}$ and $\mathbf{5})$. $\mathrm{m}_{\text {sam }}$ and $\mathrm{m}_{\text {ref }}$ are the slope of the difference $(\triangle \mathrm{OD})$ in the change in the absorption maximum wavelength of DPBF (around 410 $\mathrm{nm}$ ), which are plotted against the photoirradiation time, $\mathrm{L}_{\mathrm{ref}}$ and $\mathrm{L}_{\mathrm{sam}}$ are the light harvesting efficiency, which is given by $\mathrm{L}=1-10^{-\mathrm{A}}$ (" $\mathrm{A}$ " is the absorbance at the photoirradiation wavelength $532 \mathrm{~nm}$ ). 

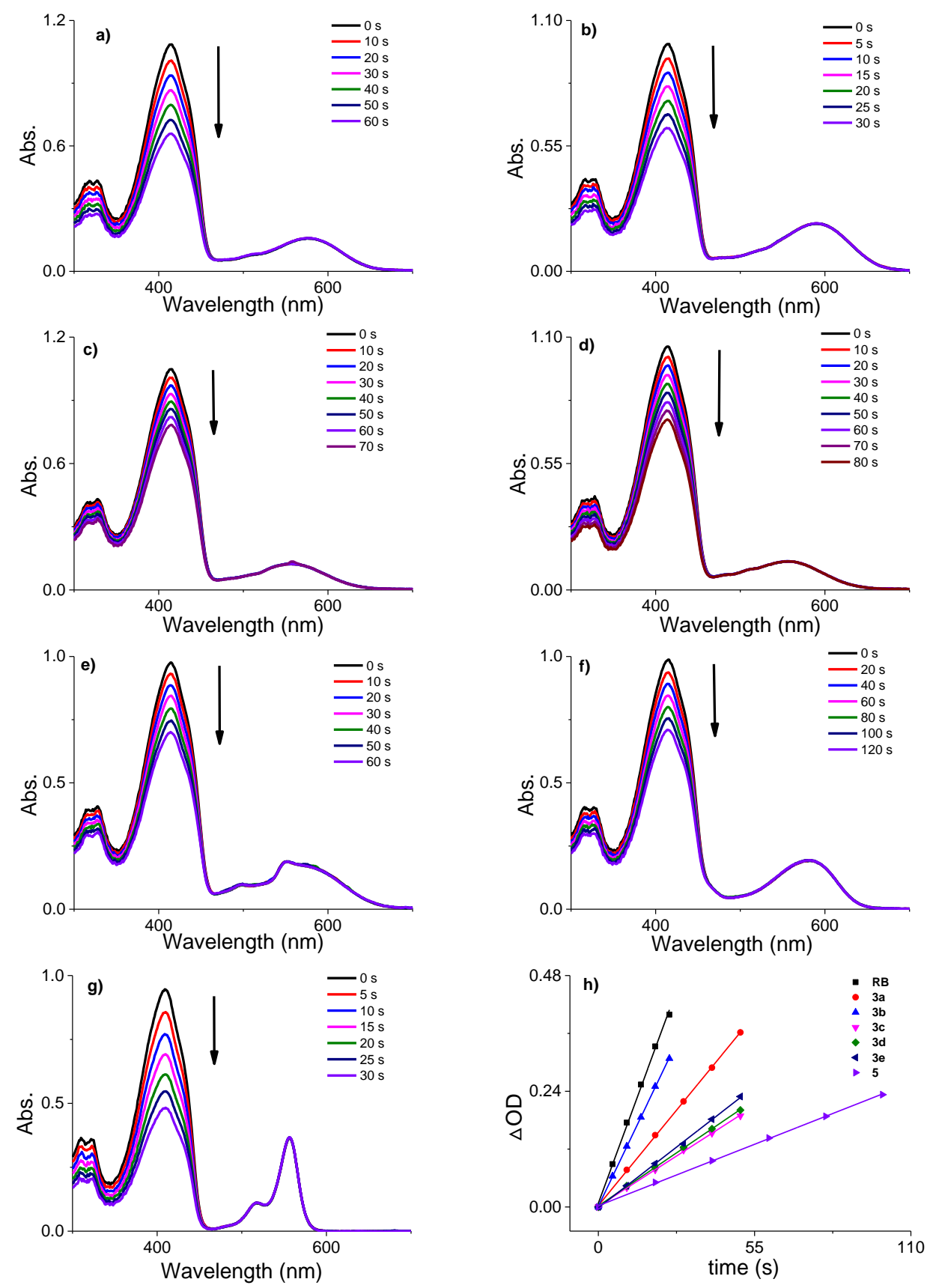

Figure S21. Absorption spectra of DPBF (initial absorbance around 1.0 at $410 \mathrm{~nm}$ ) upon irradiation in the presence of BODIPY 3a with initial absorbance around 0.1 at $532 \mathrm{~nm}$. a) 3a for $60 \mathrm{~s}$ (recorded at $10 \mathrm{~s}$ intervals) in chloroform; b) $3 \mathbf{b}$ for $30 \mathrm{~s}$ (recorded at $5 \mathrm{~s}$ intervals) in chloroform; c) $3 \mathrm{c}$ for $60 \mathrm{~s}$ (recorded at $10 \mathrm{~s}$ intervals) in chloroform; d) 3d for $60 \mathrm{~s}$ (recorded at $10 \mathrm{~s}$ intervals) in chloroform; e) 3e for $60 \mathrm{~s}$ (recorded at 10 $\mathrm{s}$ intervals) in chloroform; f) $\mathbf{5}$ for $120 \mathrm{~s}$ (recorded at $20 \mathrm{~s}$ intervals) in chloroform; $\mathrm{g}$ ) RB for $30 \mathrm{~s}$ (recorded at $5 \mathrm{~s}$ intervals) in methanol; h) plot of change in absorbance of DPBF at $410 \mathrm{~nm}$ vs irradiation time in the presence of BODIPY 3a-e and $\mathbf{5}$ in chloroform against $\mathbf{R B}$ as the standard in methanol. 

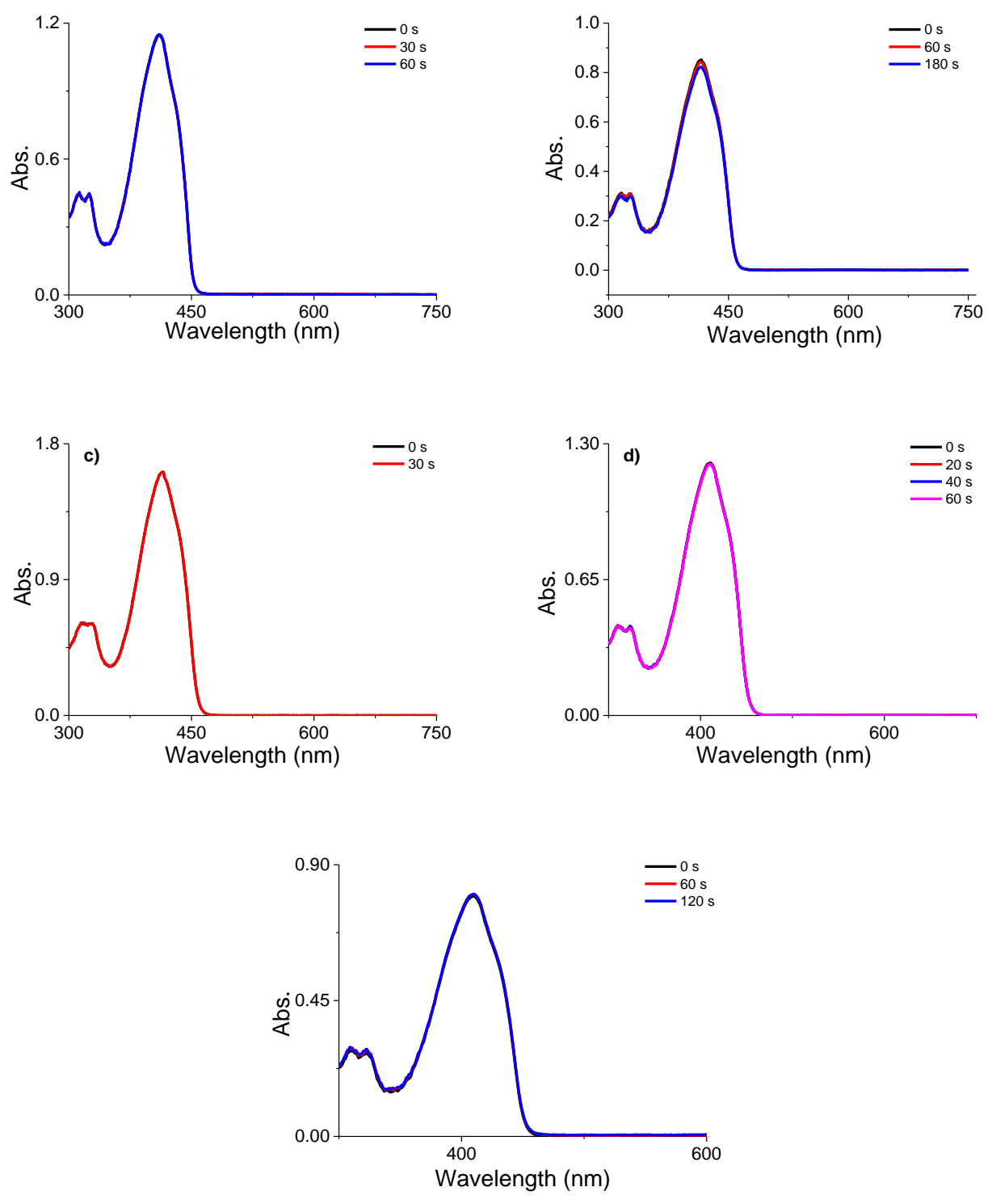

Figure S22. Control experiments: absorption spectra of DPBF (initial absorbance around 1.0 at $415 \mathrm{~nm}$ ) upon irradiation in different solvents; a) in hexane for irradiation $60 \mathrm{~s}$; b) in toluene for irradiation $180 \mathrm{~s}$; c) in chloroform for irradiation $30 \mathrm{~s}$; d) in acetonitrile for irradiation $60 \mathrm{~s}$; e) in methanol for irradiation $120 \mathrm{~s}$. 
9. ${ }^{1} \mathrm{H},{ }^{13} \mathrm{C}$ NMR and HRMS spectra for all new compounds

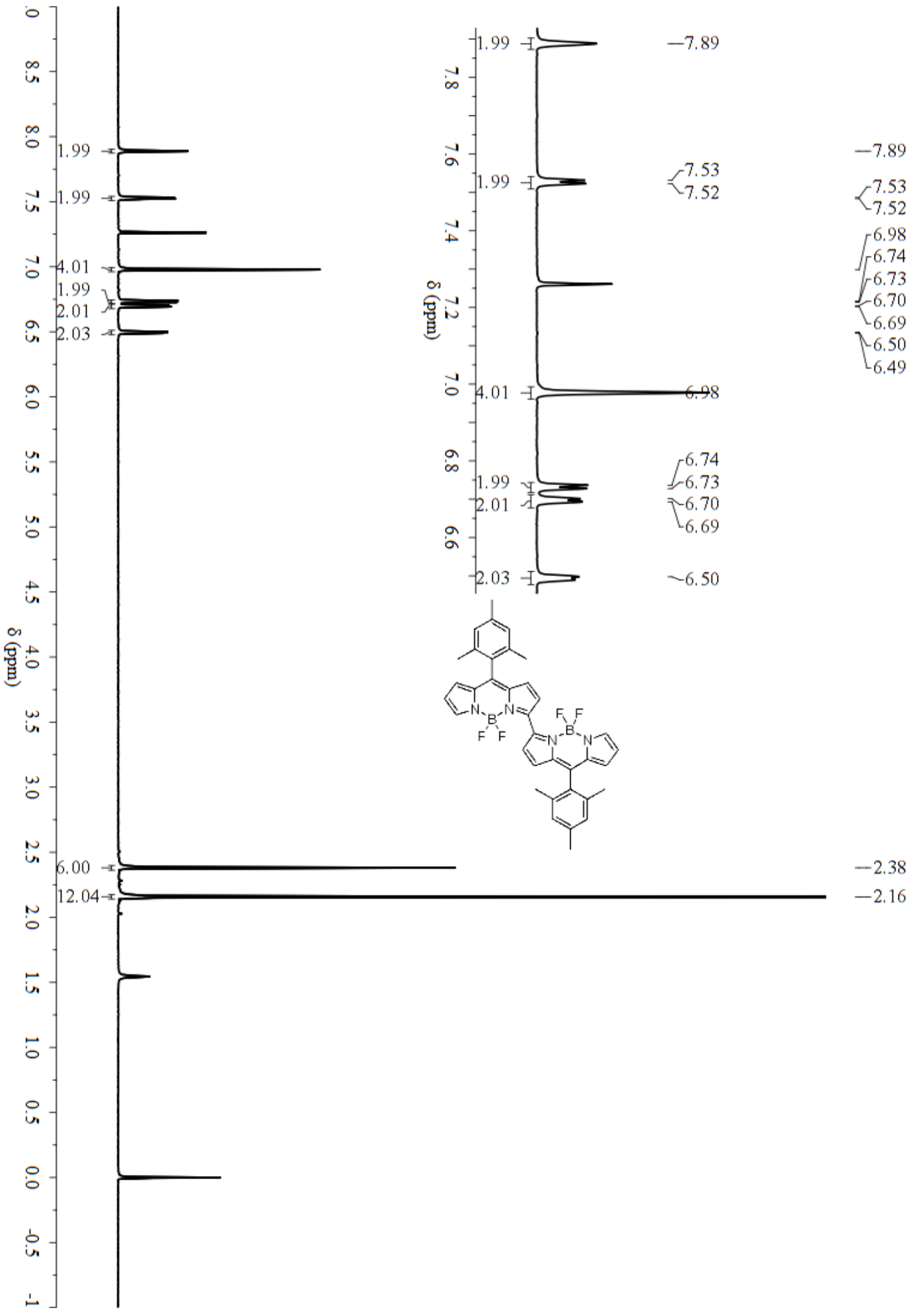

${ }^{1} \mathrm{H}$ NMR spectrum of $\mathbf{2 a}\left(\mathrm{CDCl}_{3}, 500 \mathrm{MHz}\right)$ 


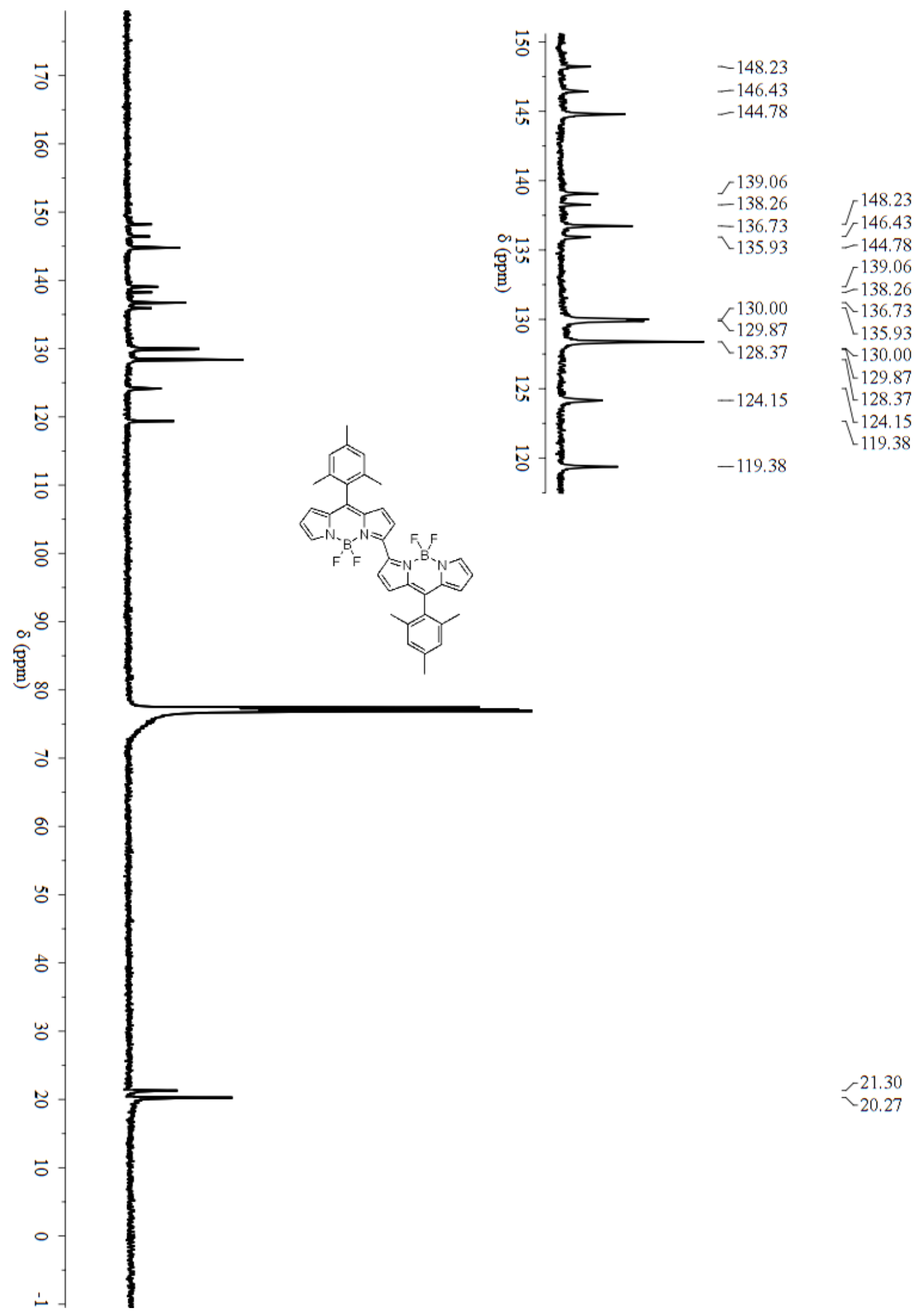

${ }^{13} \mathrm{C}$ NMR spectrum of $\mathbf{2 a}\left(\mathrm{CDCl}_{3}, 126 \mathrm{MHz}\right)$ 


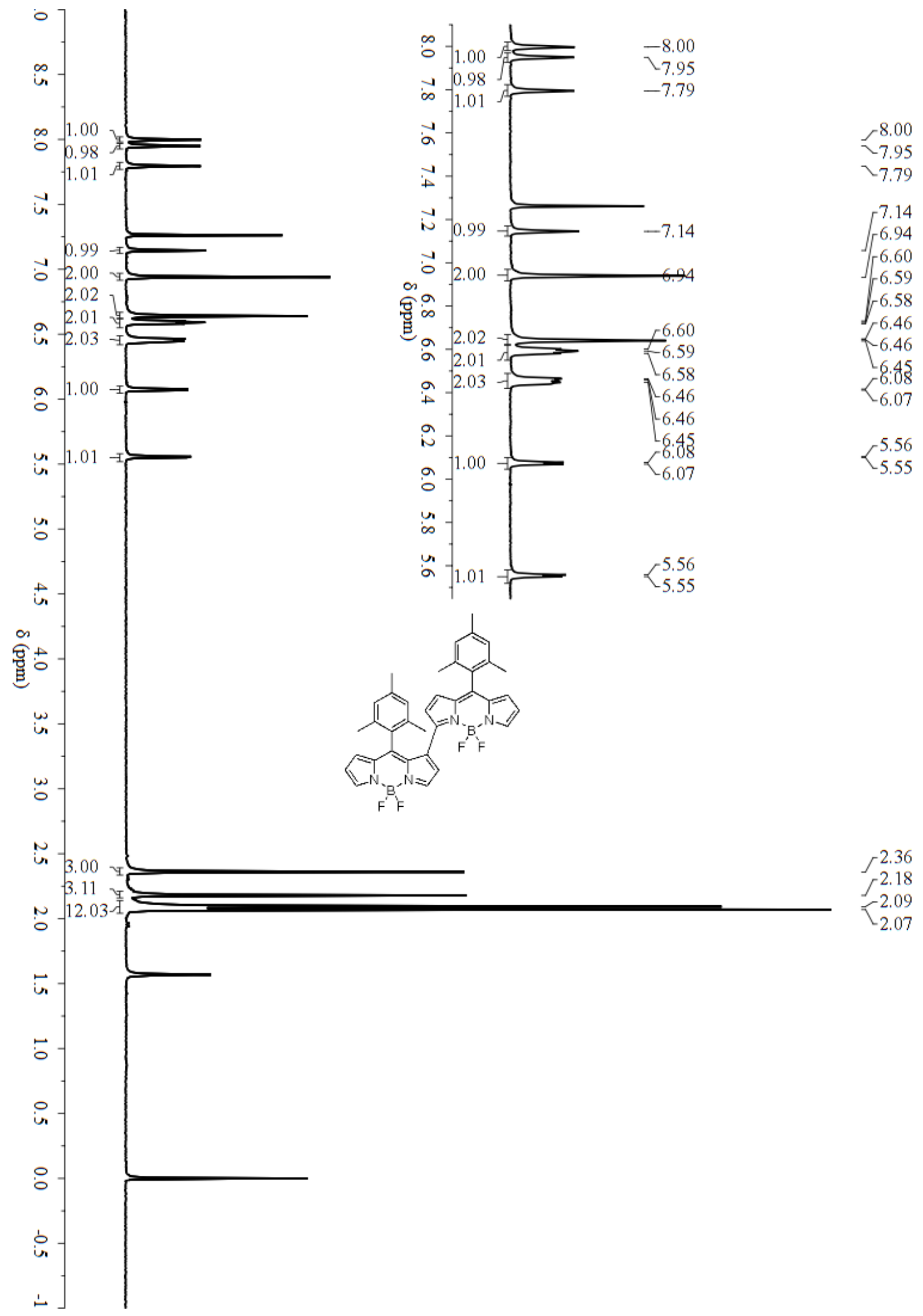

${ }^{1} \mathrm{H}$ NMR spectrum of $\mathbf{3 a}\left(\mathrm{CDCl}_{3}, 500 \mathrm{MHz}\right)$ 


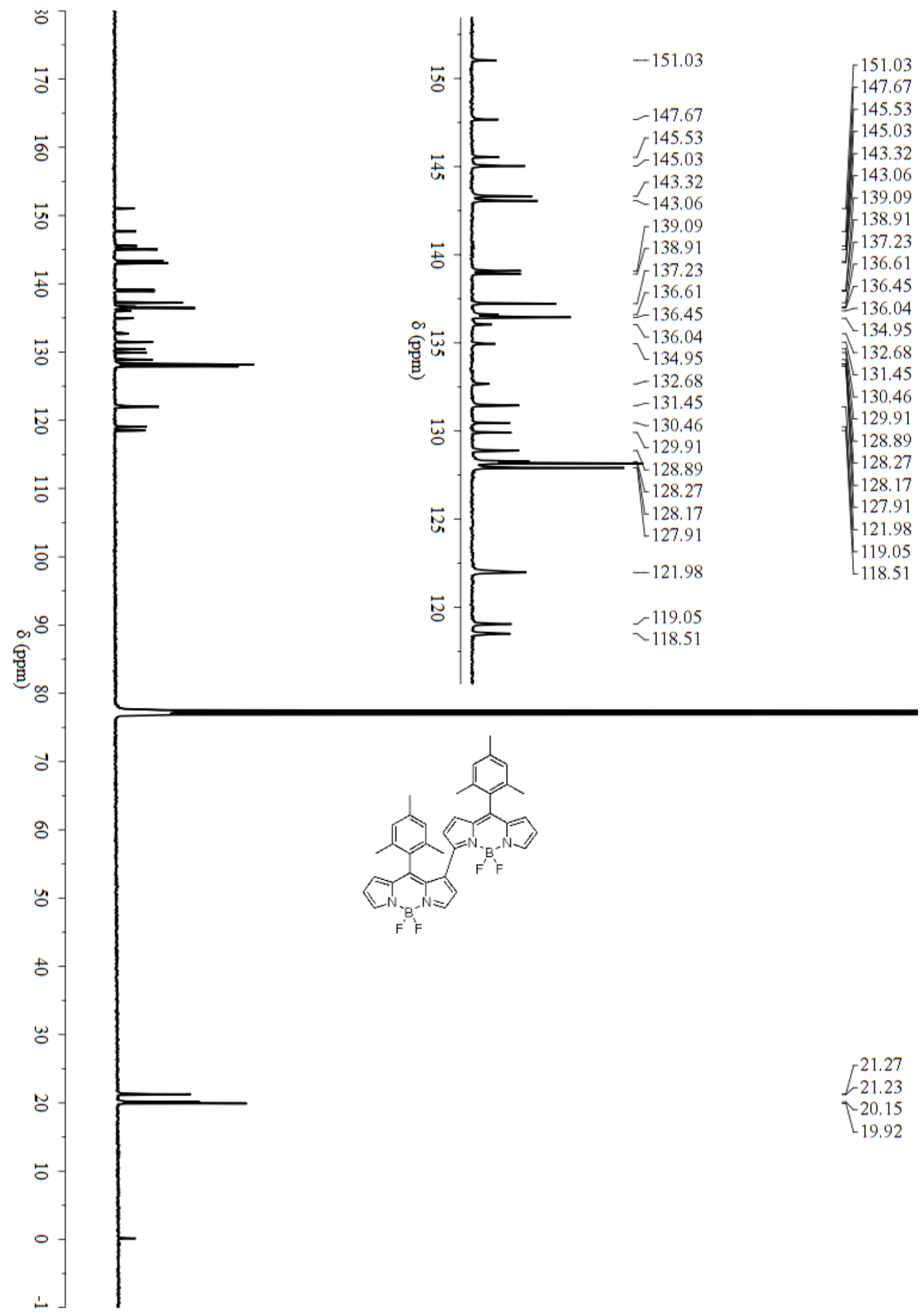

${ }^{13} \mathrm{C}$ NMR spectrum of $\mathbf{3 a}\left(\mathrm{CDCl}_{3}, 126 \mathrm{MHz}\right)$ 


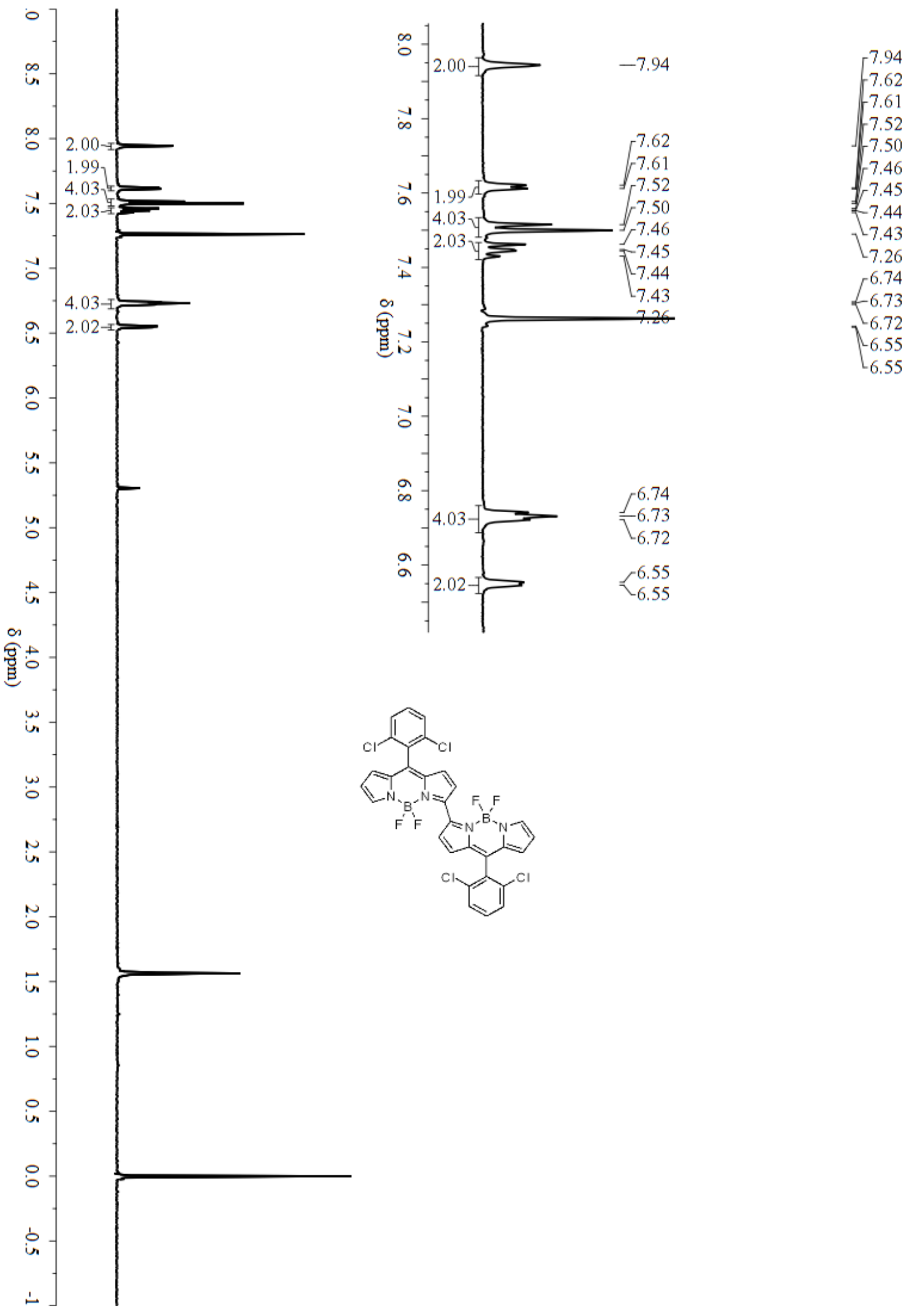

${ }^{1} \mathrm{H}$ NMR spectrum of $\mathbf{2 b}$ in $\left(\mathrm{CDCl}_{3}, 500 \mathrm{MHz}\right)$ 


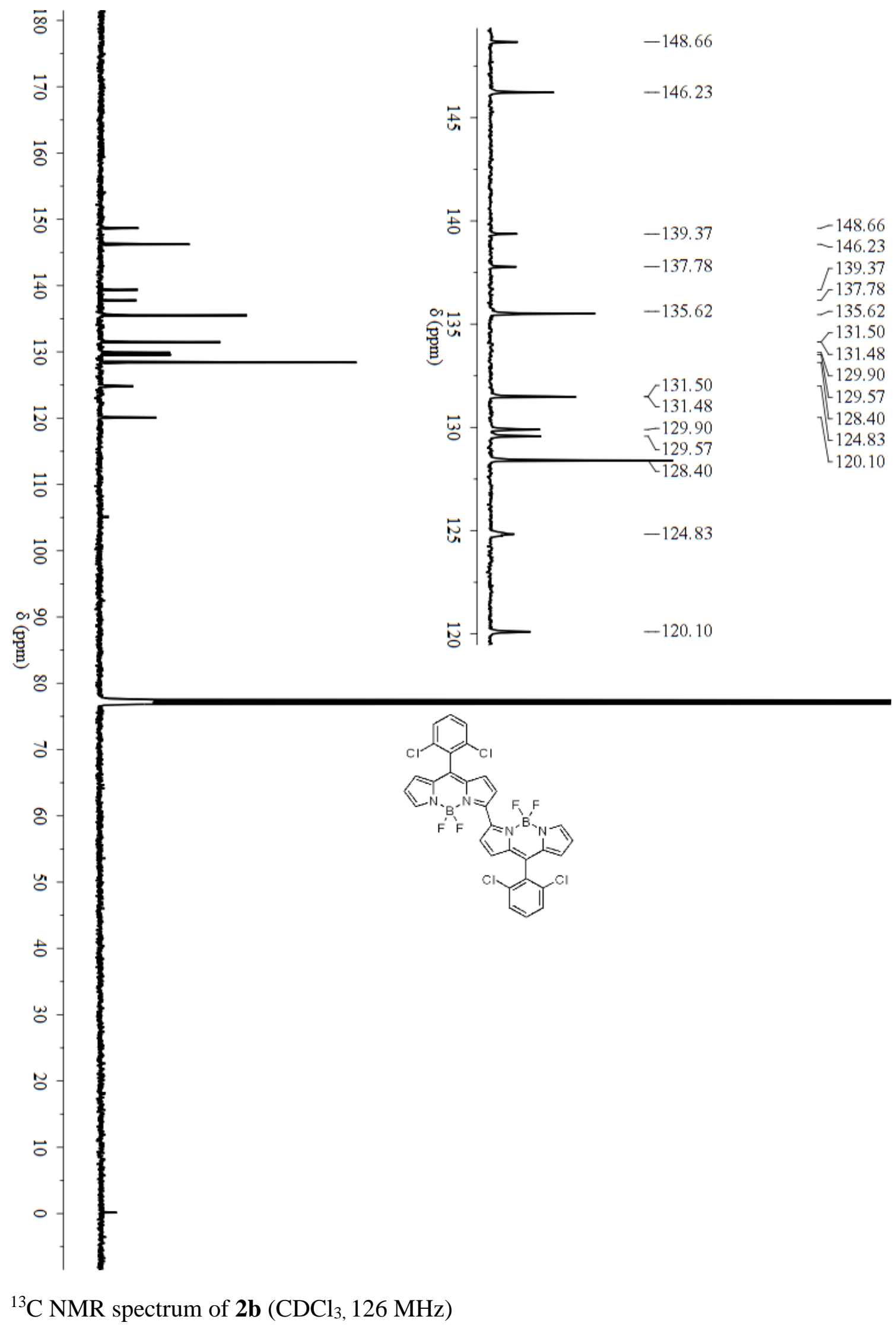




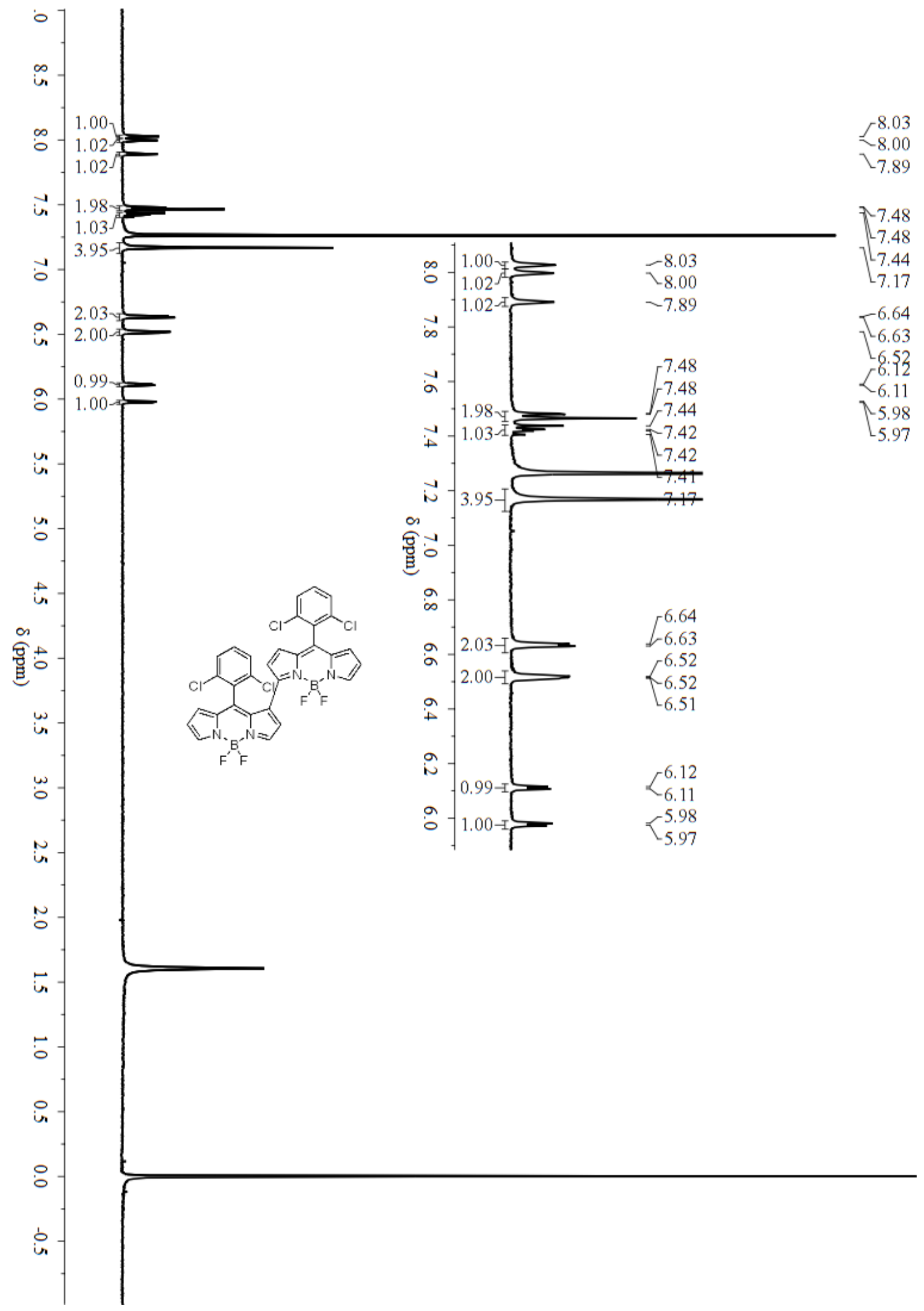

${ }^{1} \mathrm{H}$ NMR spectrum of $\mathbf{3 b}\left(\mathrm{CDCl}_{3}, 500 \mathrm{MHz}\right)$ 


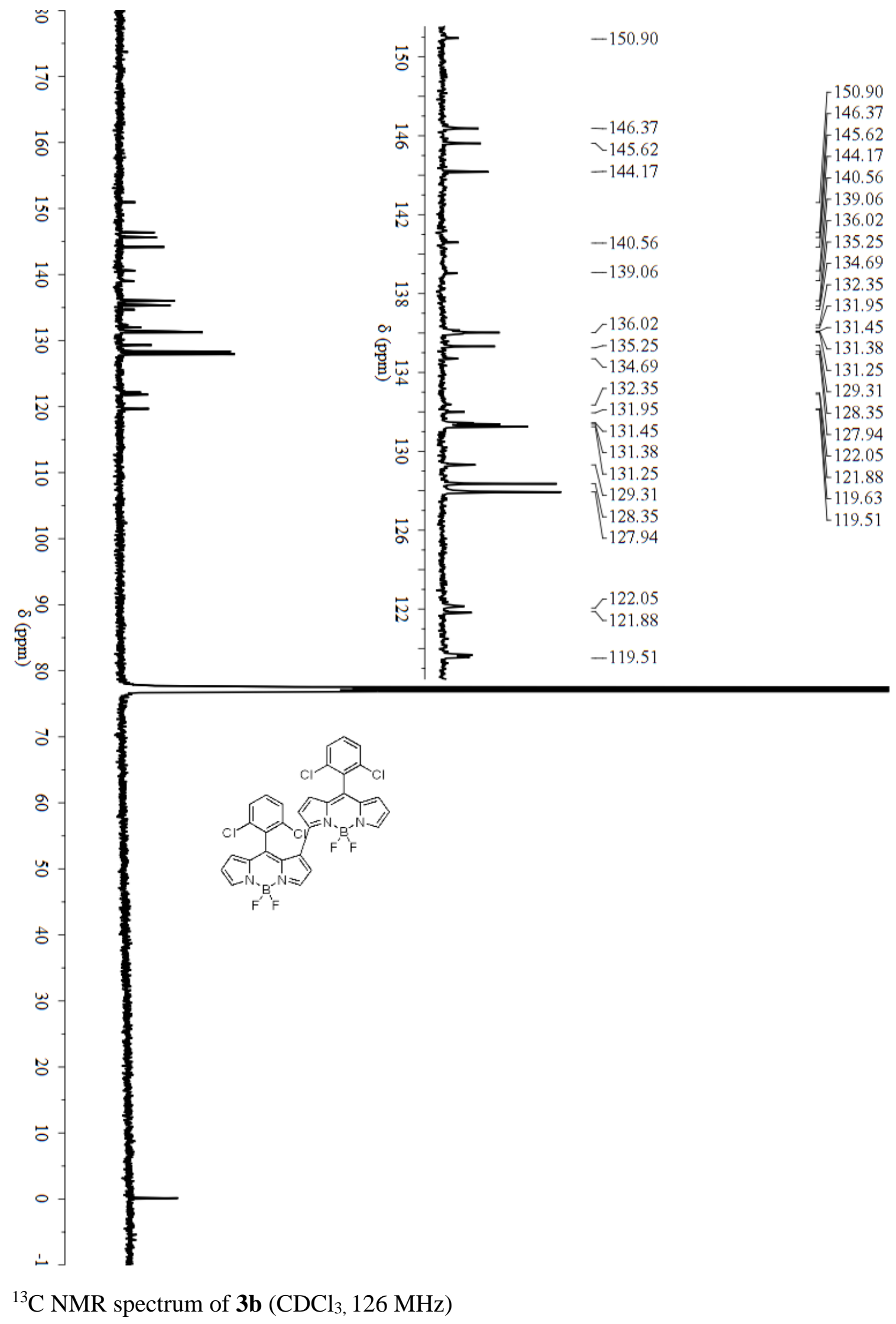




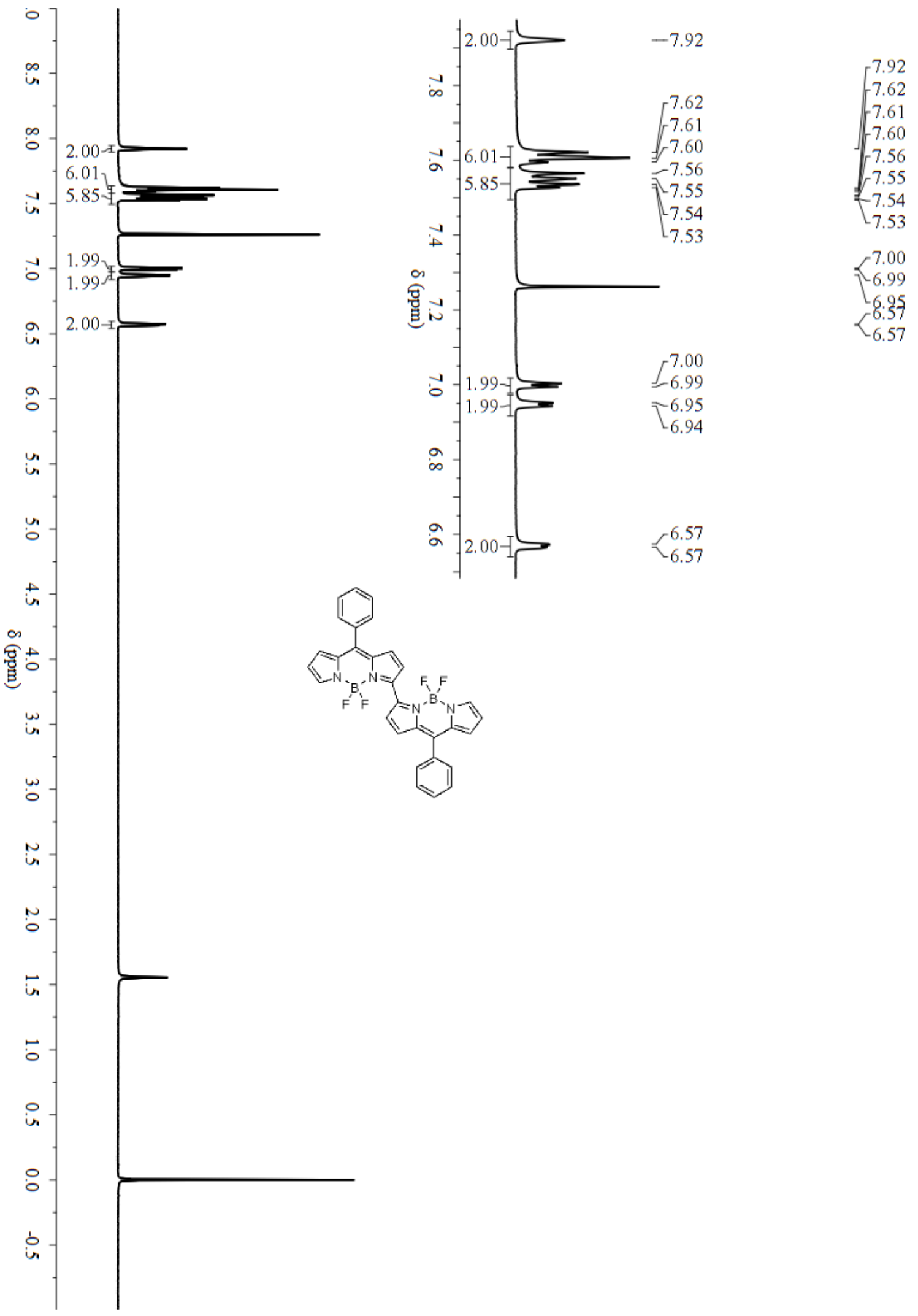

${ }^{1} \mathrm{H}$ NMR spectrum of $\mathbf{2 c}\left(\mathrm{CDCl}_{3}, 500 \mathrm{MHz}\right)$ 


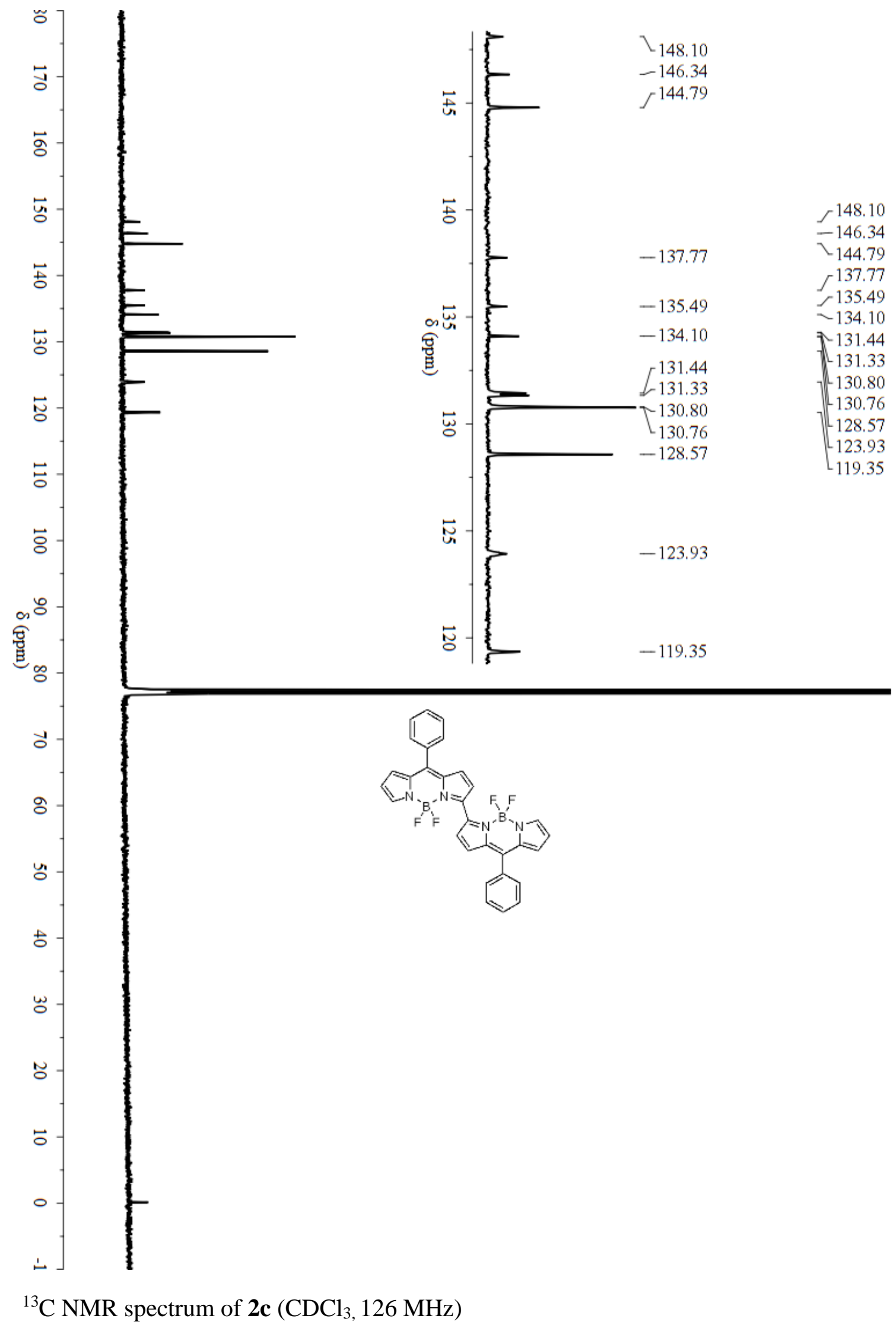




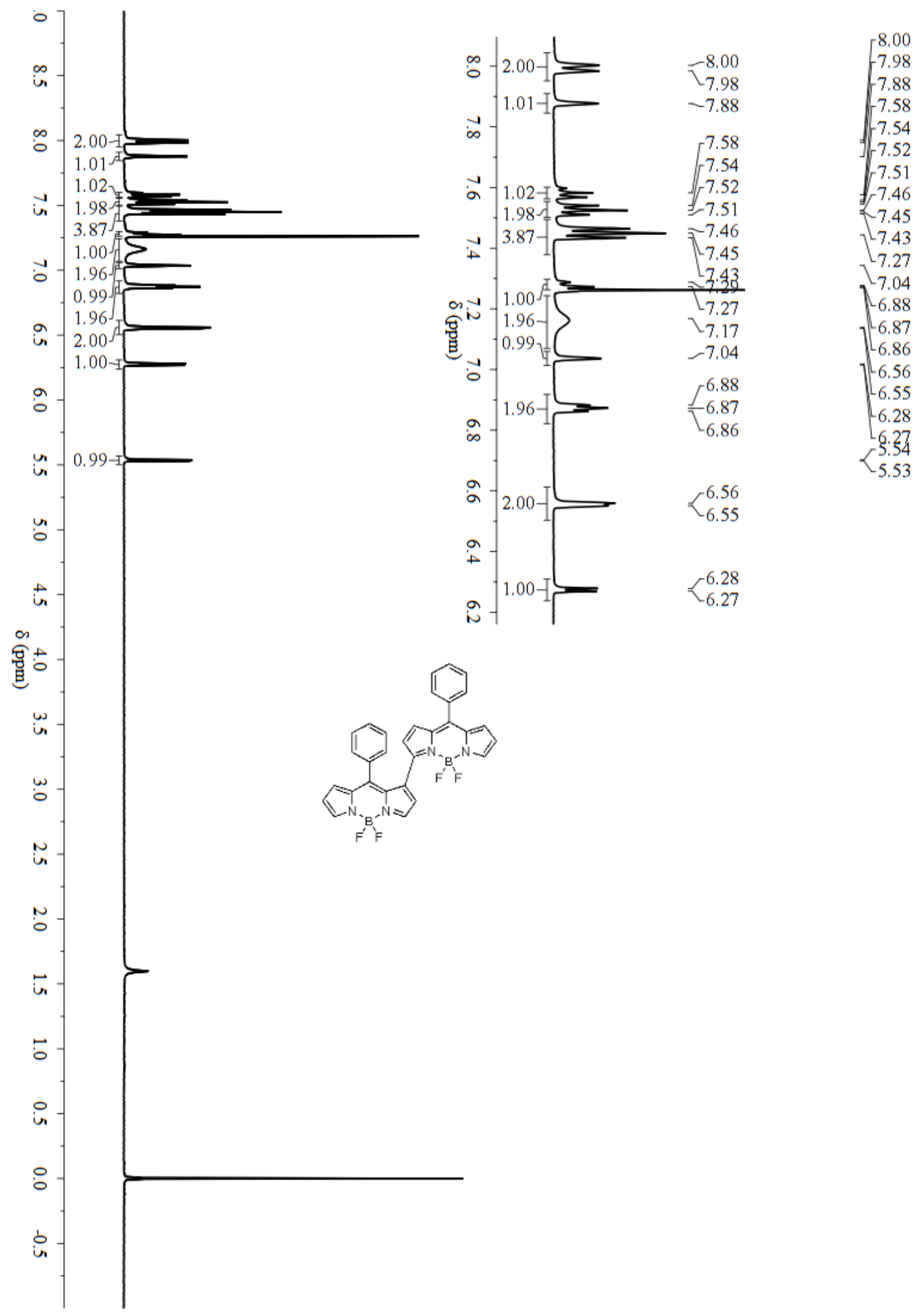

${ }^{1} \mathrm{H}$ NMR spectrum of $\mathbf{3 c}\left(\mathrm{CDCl}_{3}, 500 \mathrm{MHz}\right)$ 


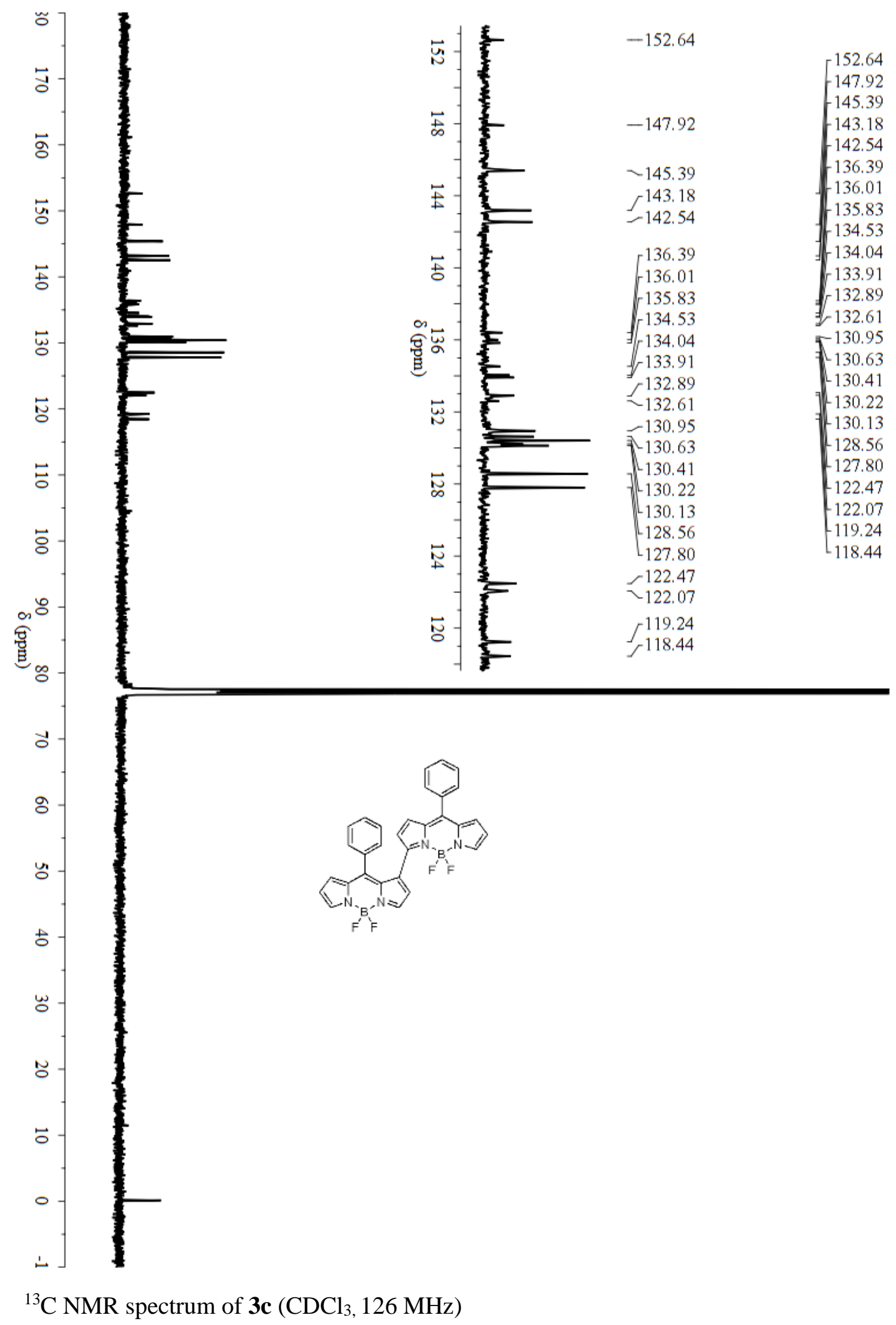



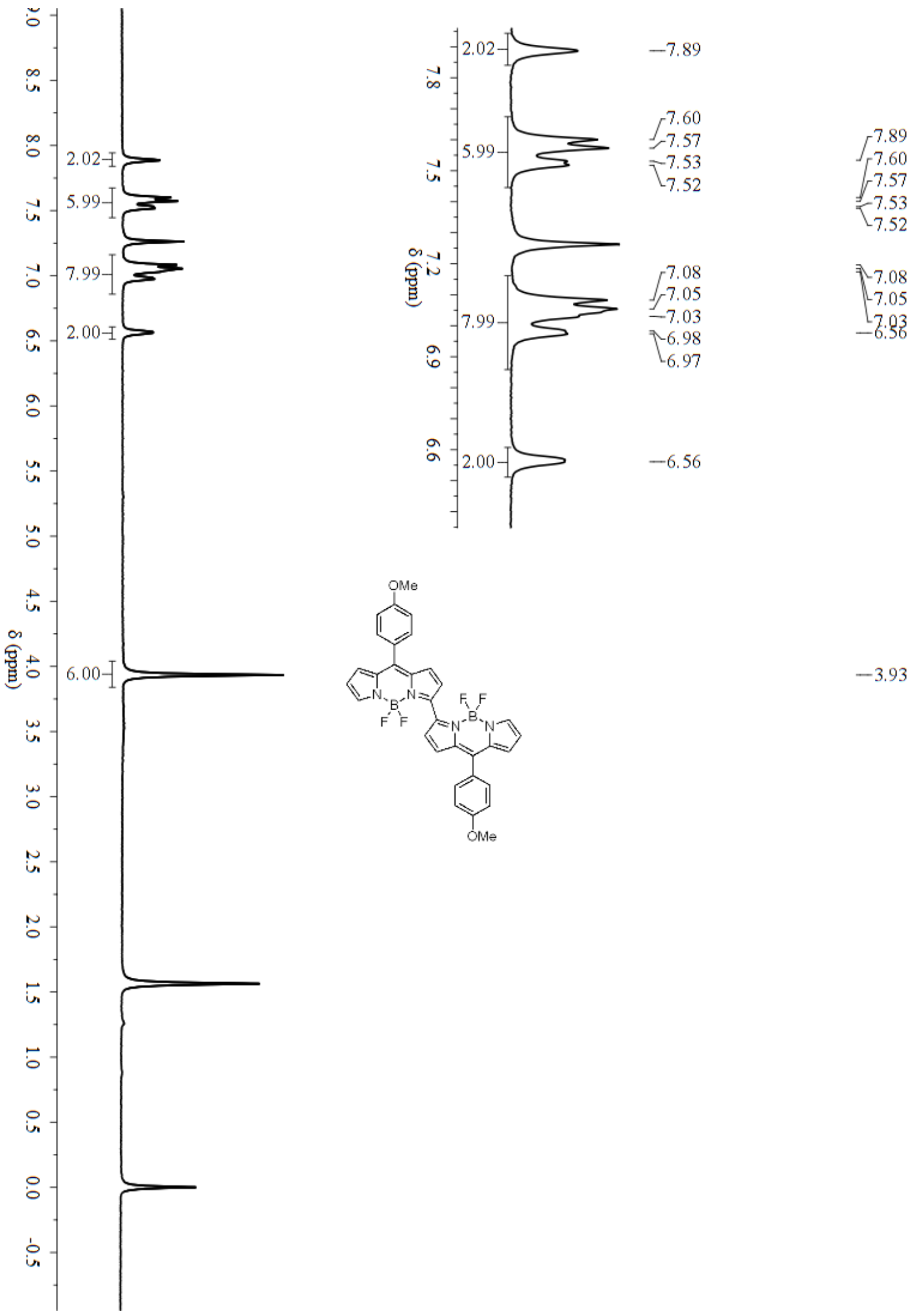

${ }^{1} \mathrm{H}$ NMR spectrum of $\mathbf{2 d}\left(\mathrm{CDCl}_{3}, 300 \mathrm{MHz}\right)$ 


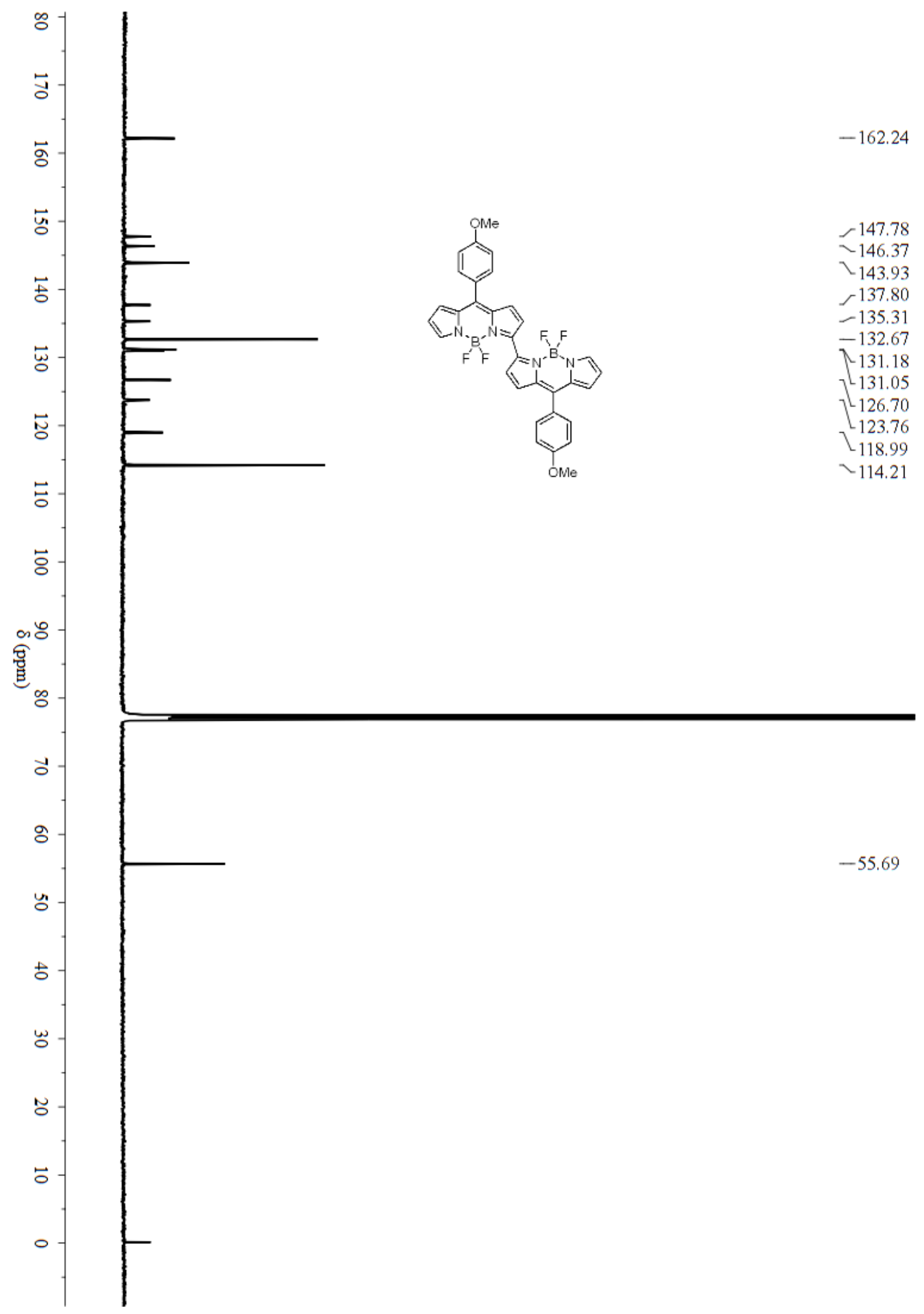

${ }^{13} \mathrm{C}$ NMR spectrum of $\mathbf{2 d}\left(\mathrm{CDCl}_{3}, 126 \mathrm{MHz}\right)$ 


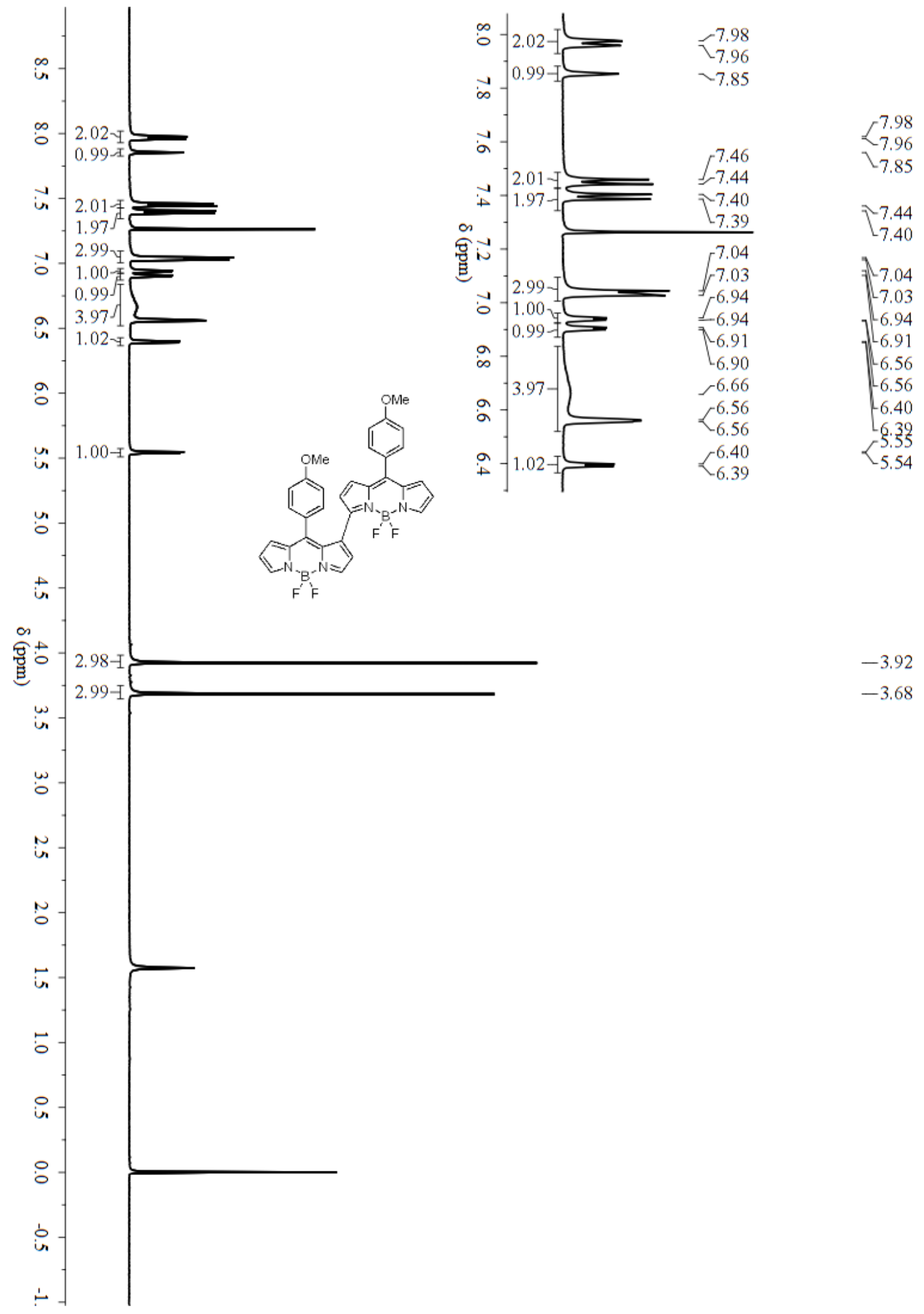

${ }^{1} \mathrm{H}$ NMR spectrum of $\mathbf{3 d}\left(\mathrm{CDCl}_{3}, 500 \mathrm{MHz}\right)$ 


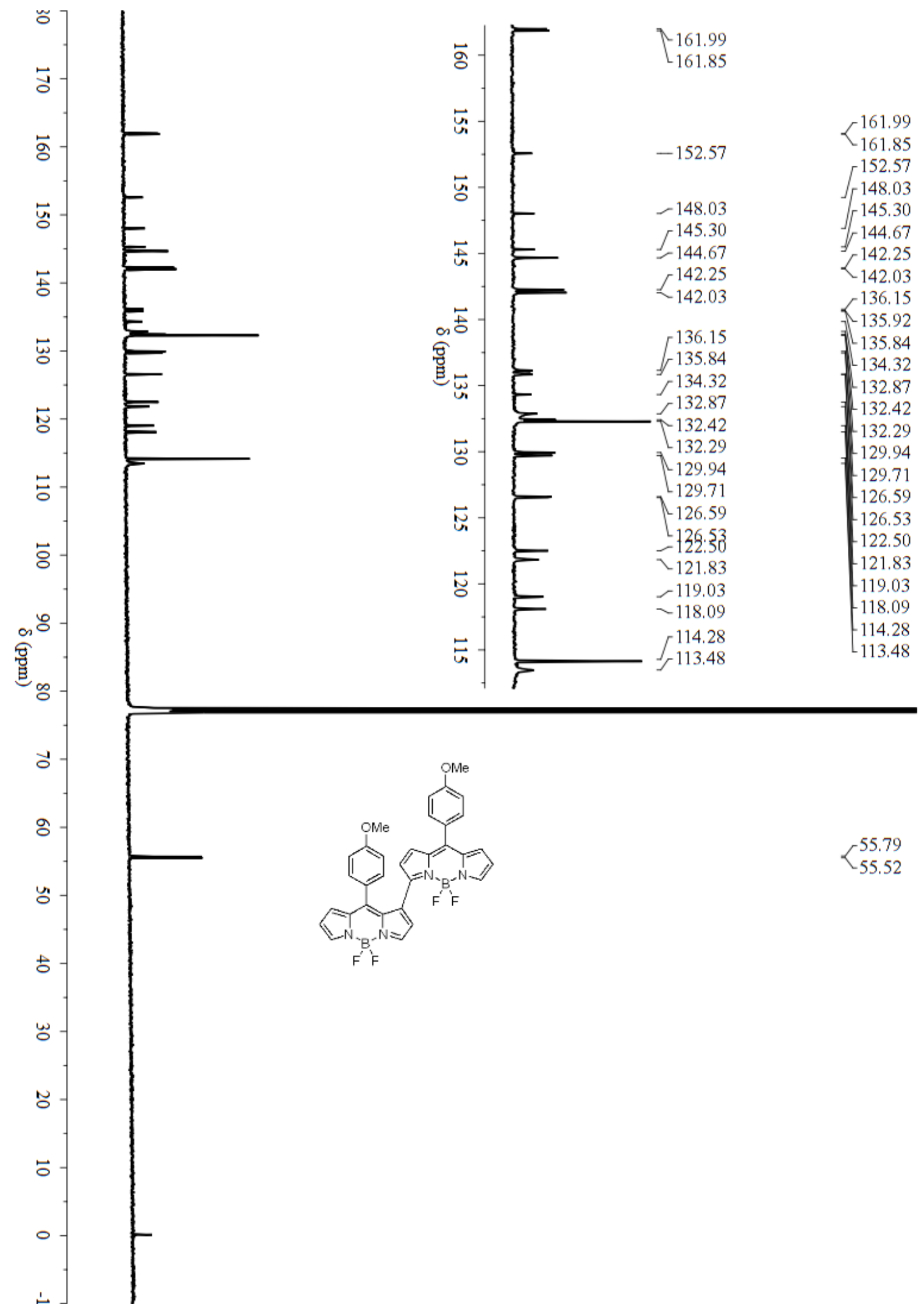

${ }^{13} \mathrm{C}$ NMR spectrum of $\mathbf{3 d}\left(\mathrm{CDCl}_{3}, 126 \mathrm{MHz}\right)$ 


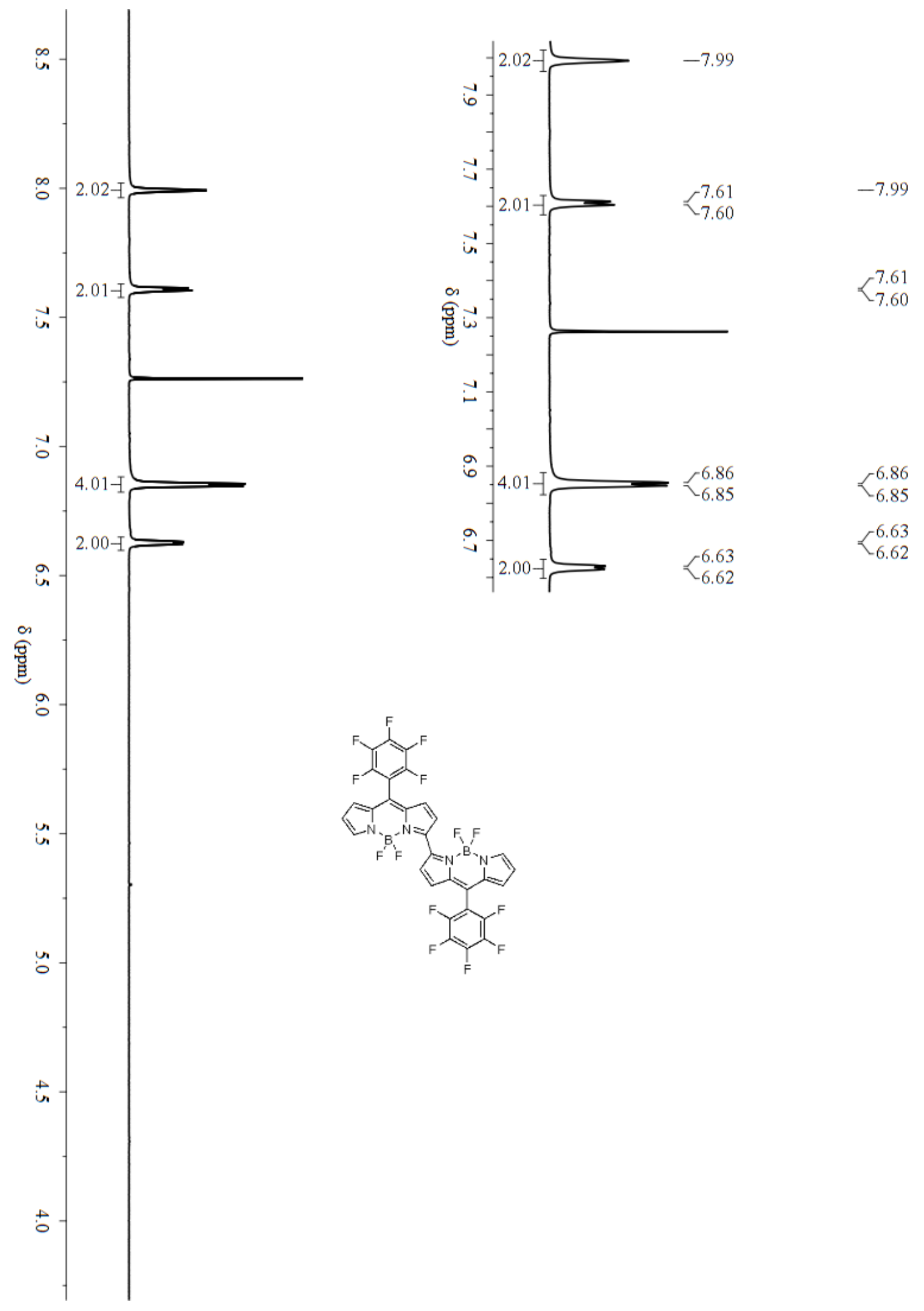

${ }^{1} \mathrm{H}$ NMR spectrum of $\mathbf{2 e}\left(\mathrm{CDCl}_{3}, 500 \mathrm{MHz}\right)$ 


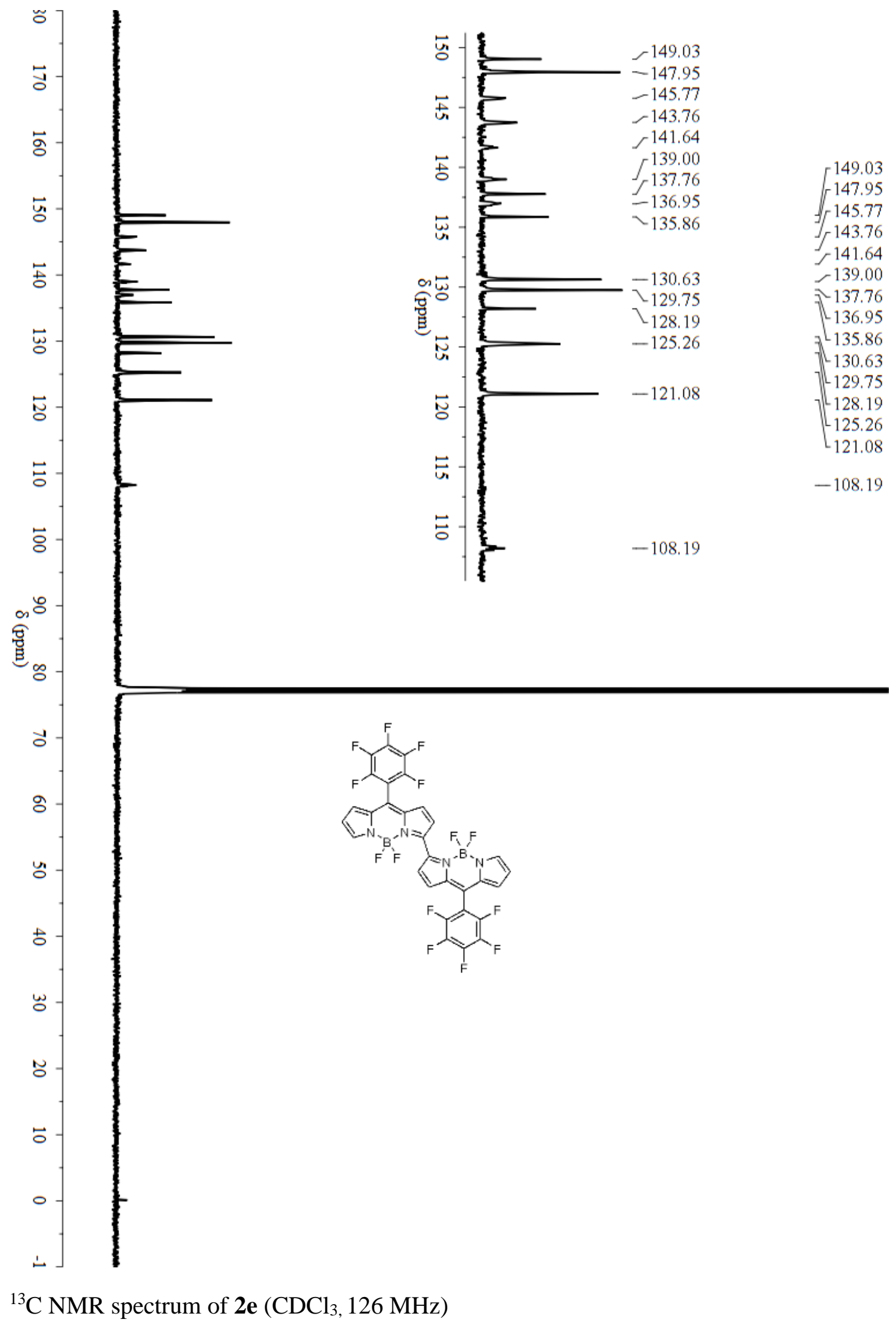




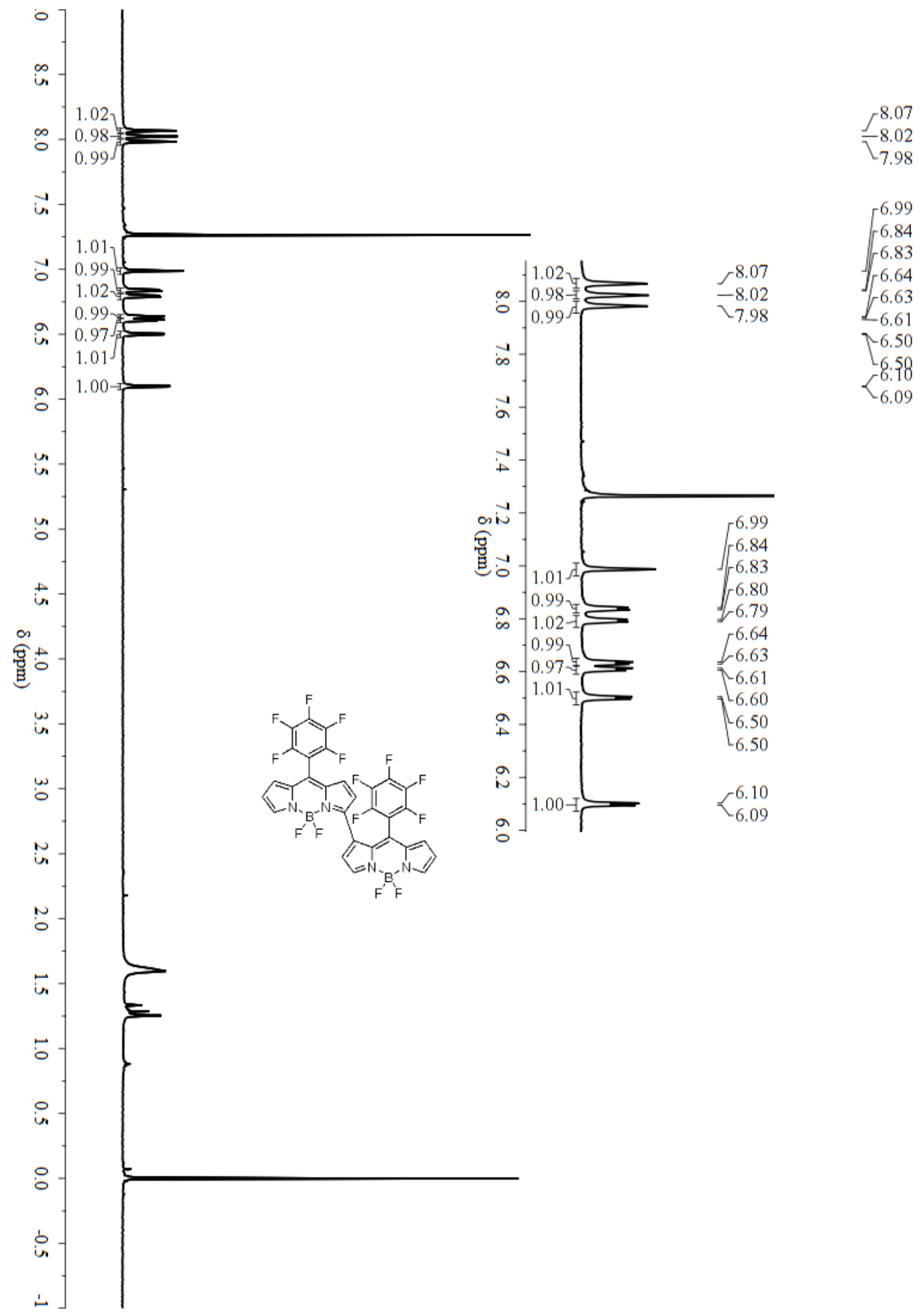

${ }^{1} \mathrm{H}$ NMR spectrum of $\mathbf{3 e}\left(\mathrm{CDCl}_{3}, 500 \mathrm{MHz}\right)$ 


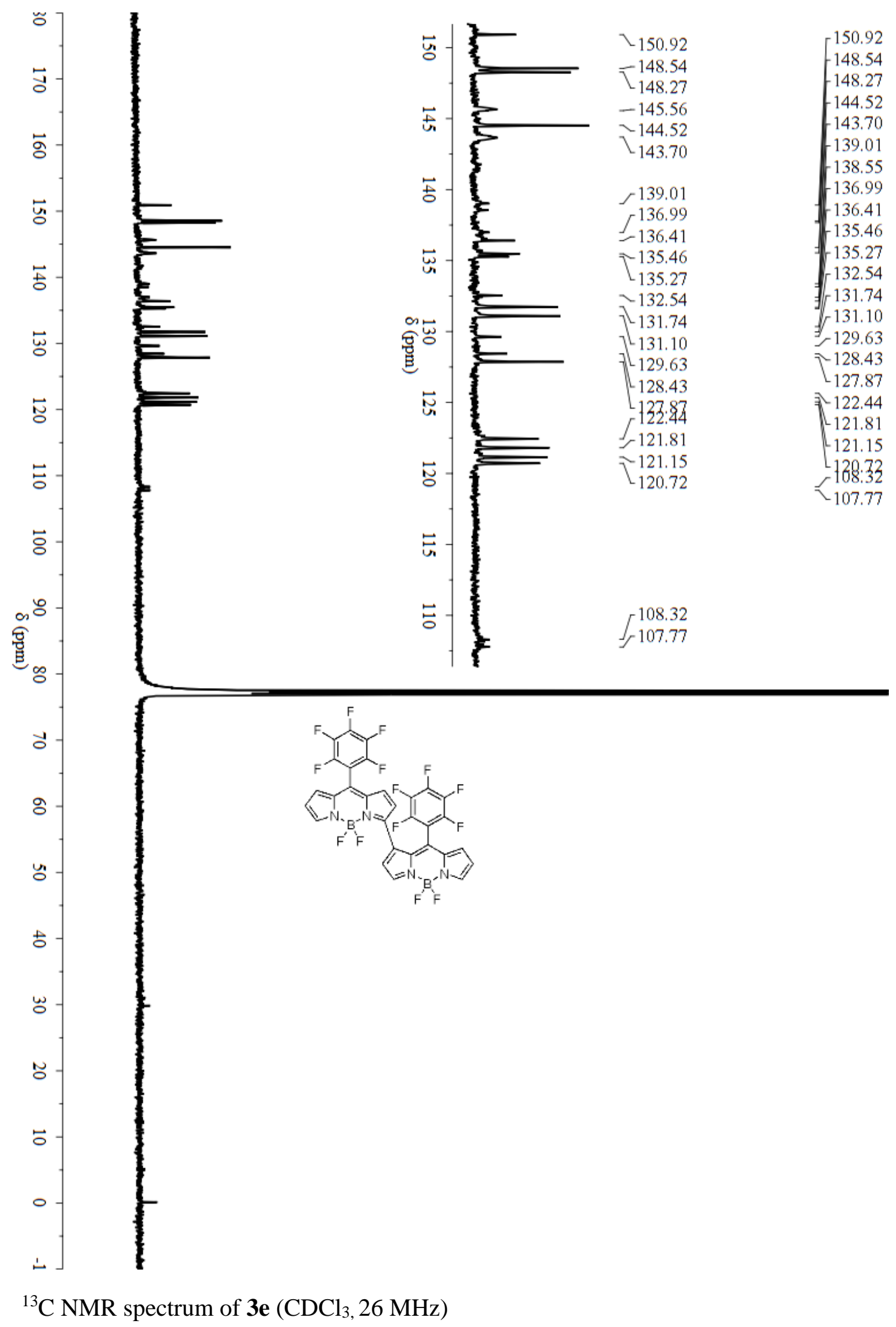




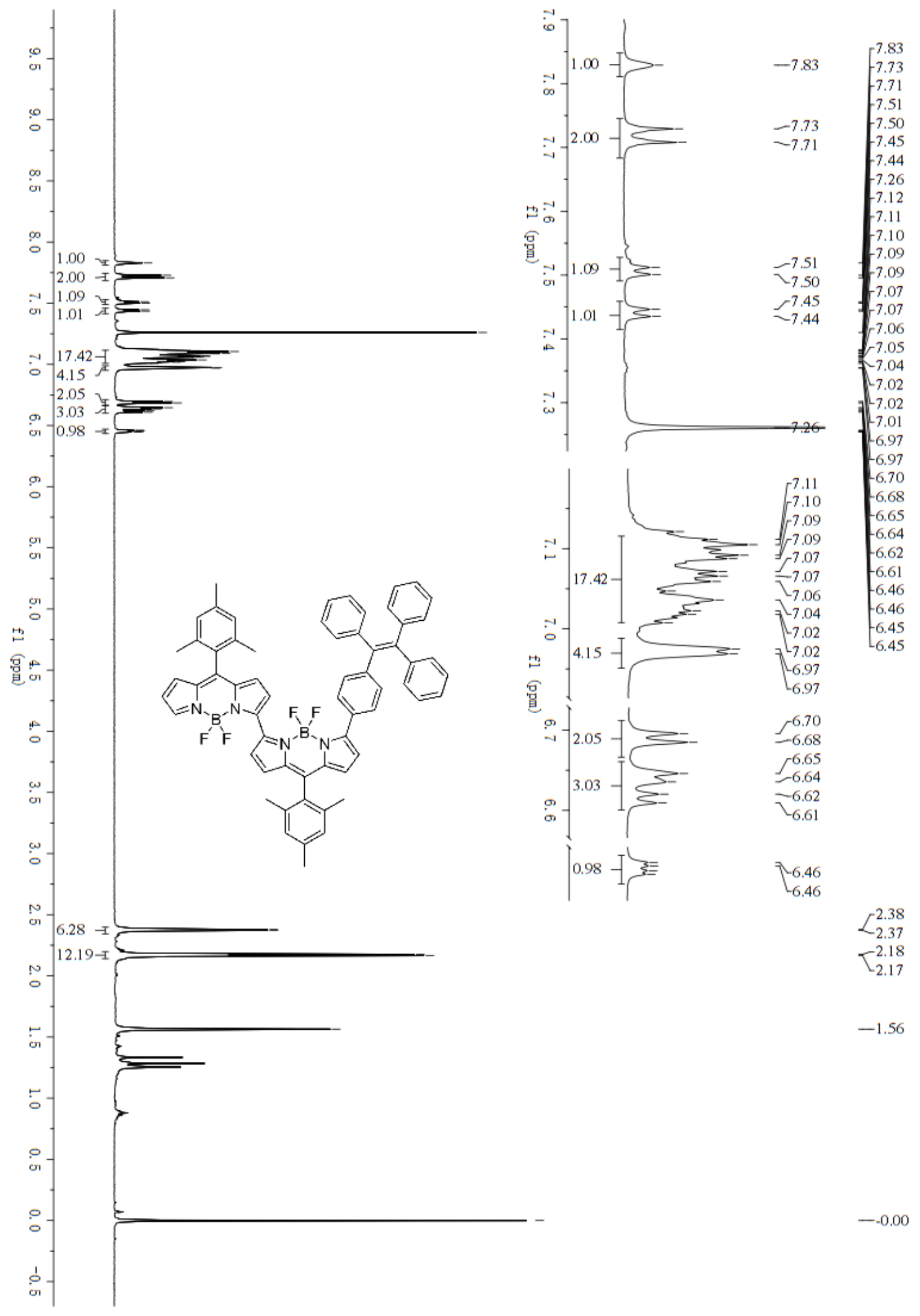

${ }^{1} \mathrm{H} \mathrm{NMR}$ spectrum of $5\left(\mathrm{CDCl}_{3}, 400 \mathrm{MHz}\right)$ 


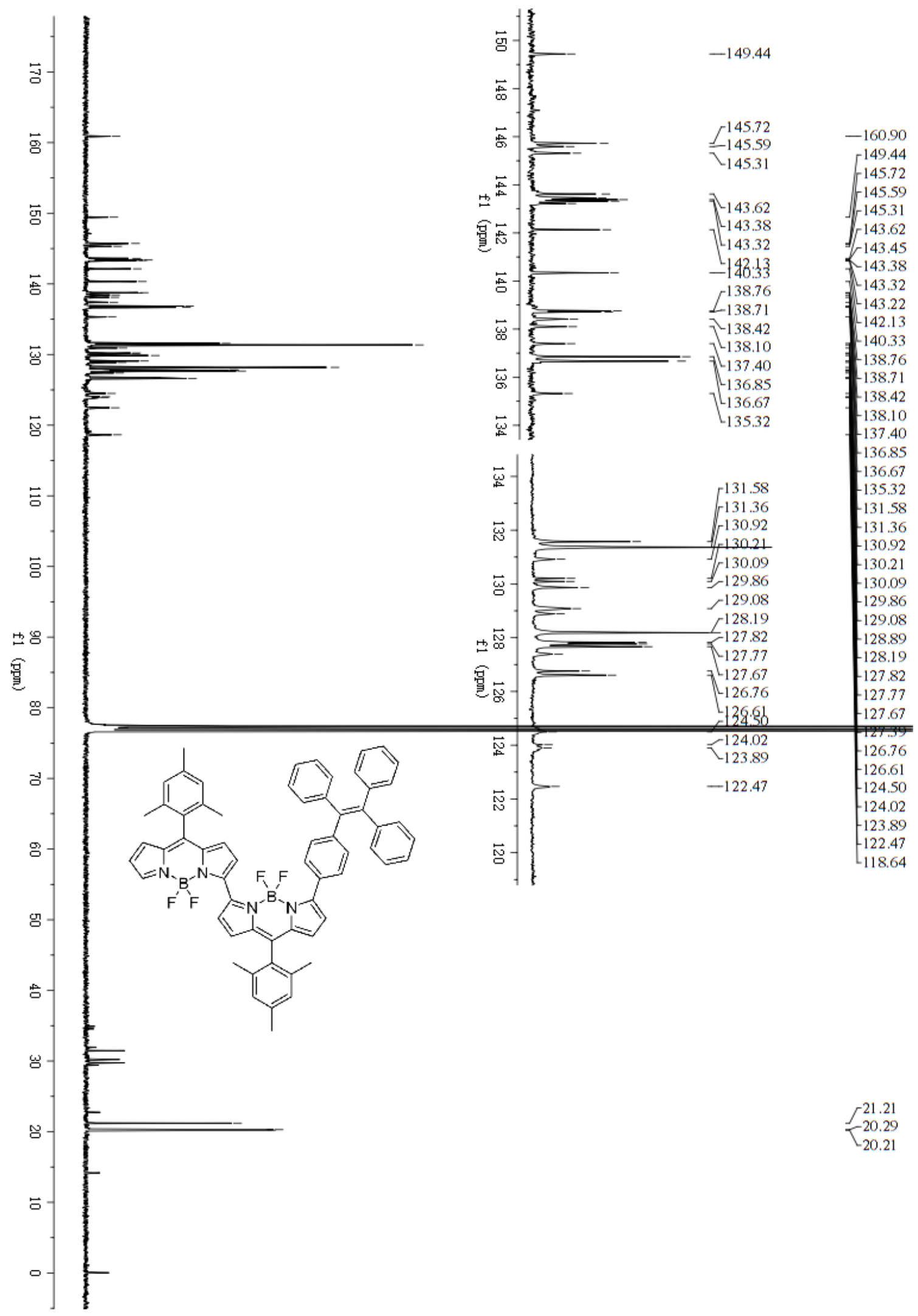

${ }^{13} \mathrm{C}$ NMR spectrum of $5\left(\mathrm{CDCl}_{3}, 101 \mathrm{MHz}\right)$ 


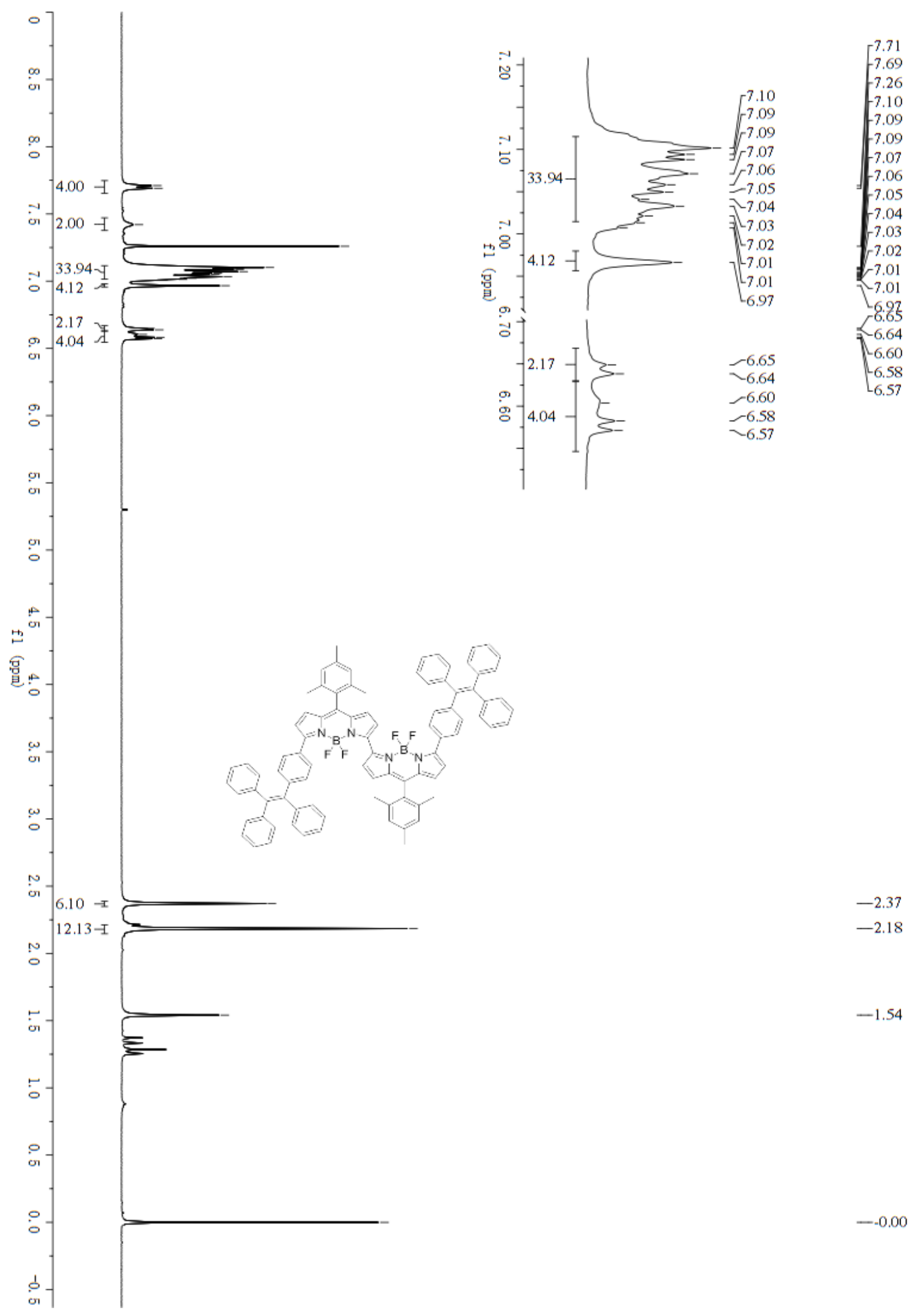

${ }^{1} \mathrm{H}$ NMR spectrum of $6\left(\mathrm{CDCl}_{3}, 400 \mathrm{MHz}\right)$ 


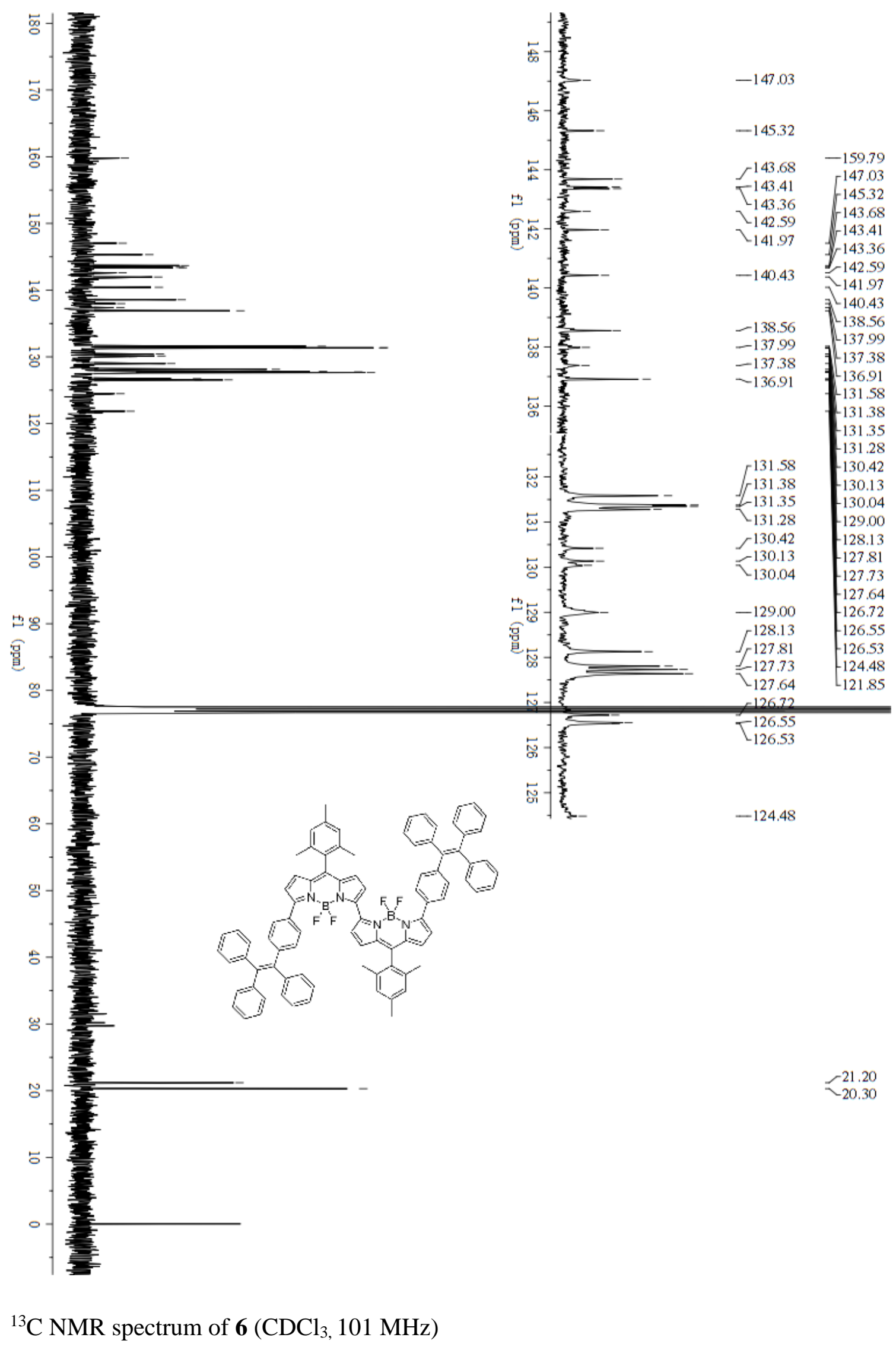




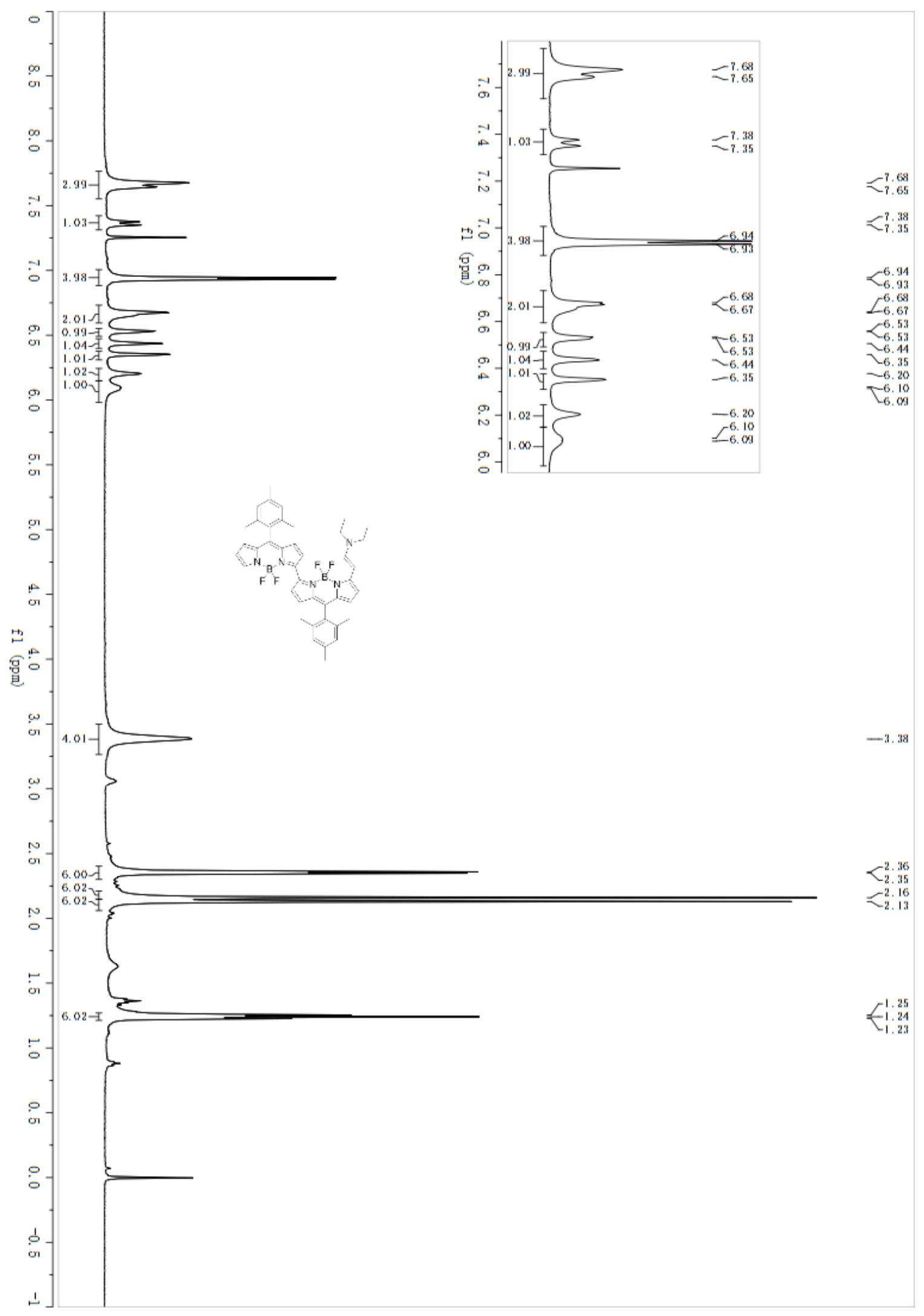

${ }^{1} \mathrm{H} \mathrm{NMR}$ spectrum of $7\left(\mathrm{CDCl}_{3}, 500 \mathrm{MHz}\right)$ 


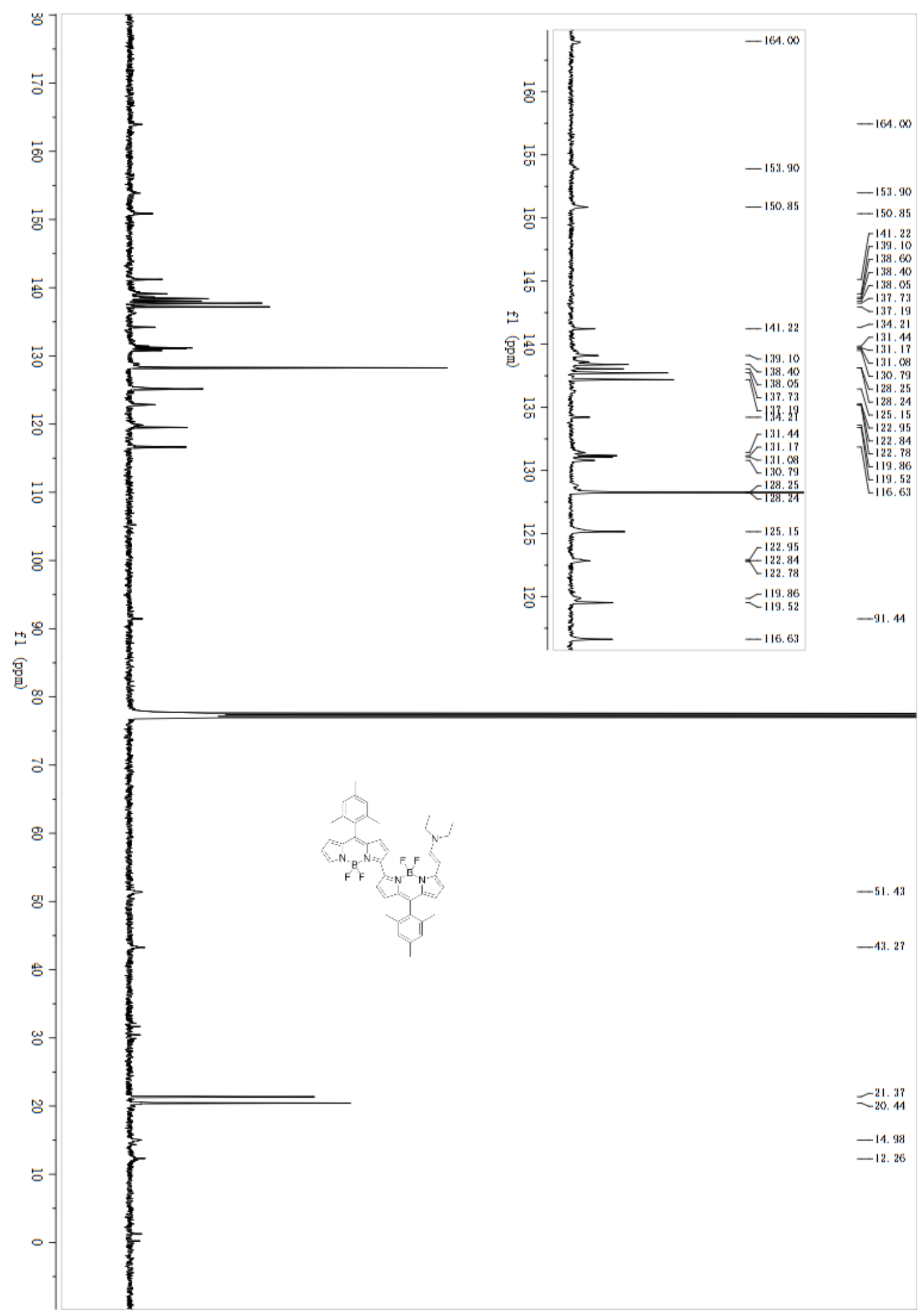

${ }^{13} \mathrm{C}$ NMR spectrum of $7\left(\mathrm{CDCl}_{3}, 126 \mathrm{MHz}\right)$ 


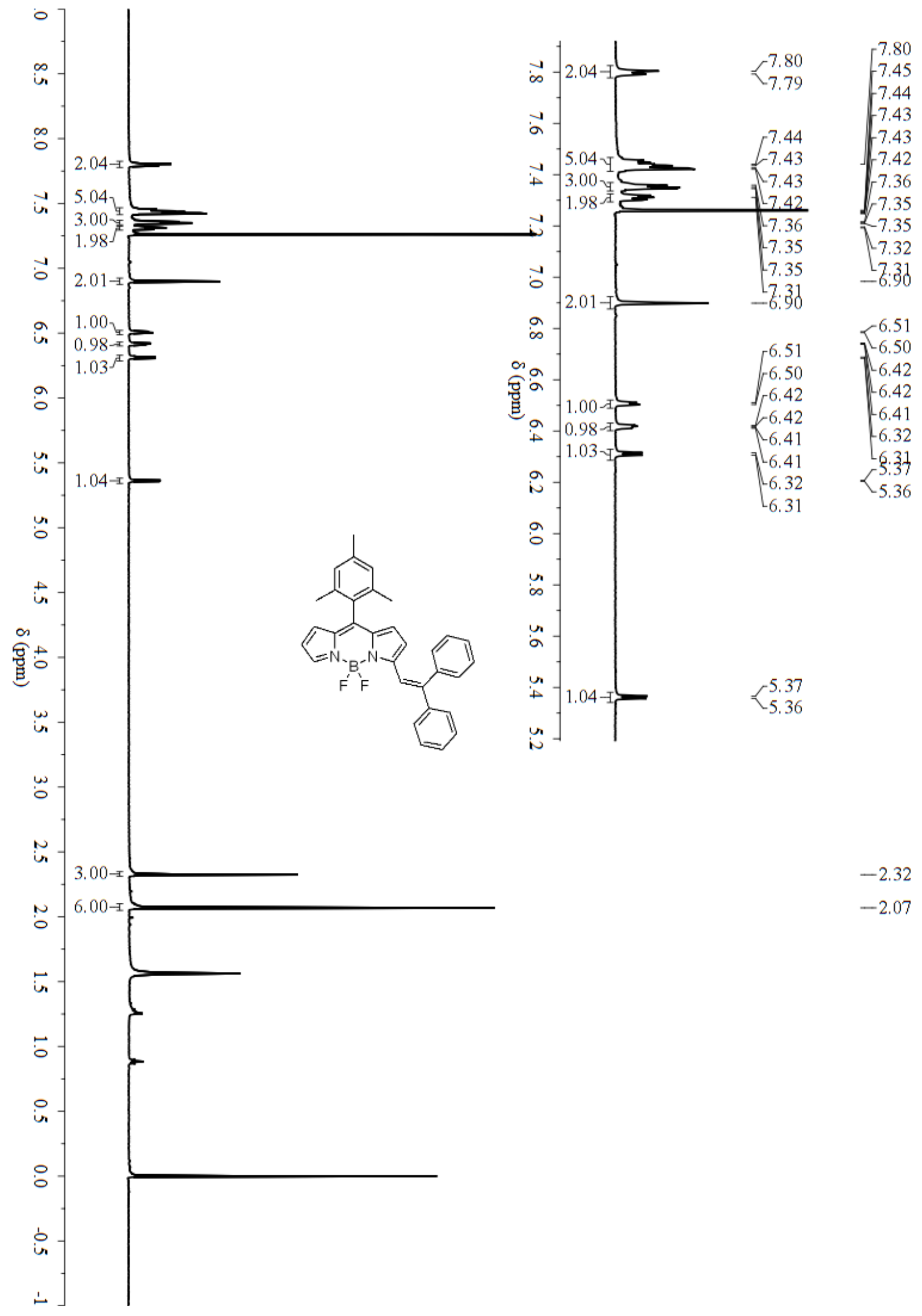

${ }^{1} \mathrm{H}$ NMR spectrum of $8\left(\mathrm{CDCl}_{3}, 500 \mathrm{MHz}\right)$ 


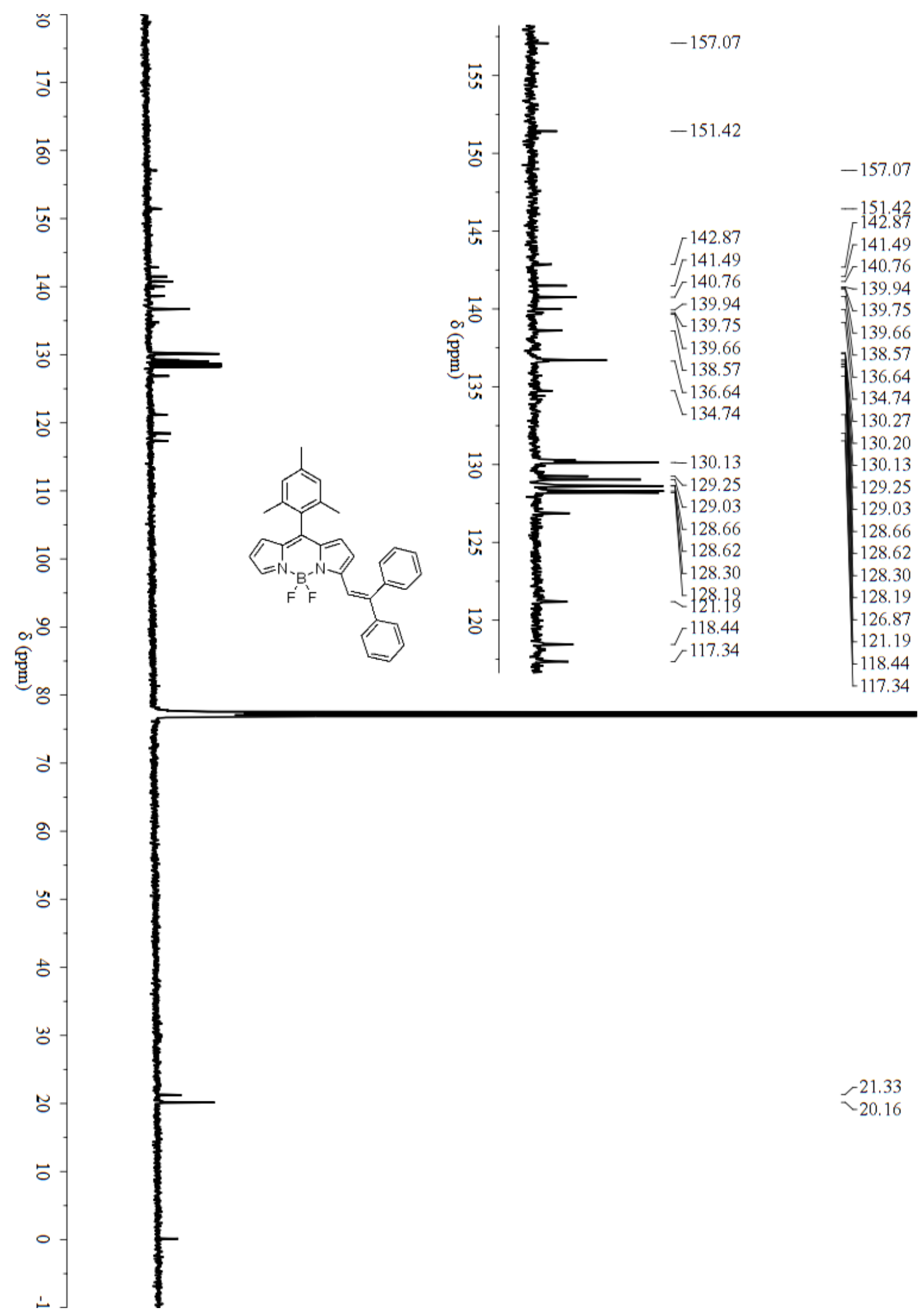

${ }^{13} \mathrm{C}$ NMR spectrum of $\mathbf{8}\left(\mathrm{CDCl}_{3}, 126 \mathrm{MHz}\right)$ 


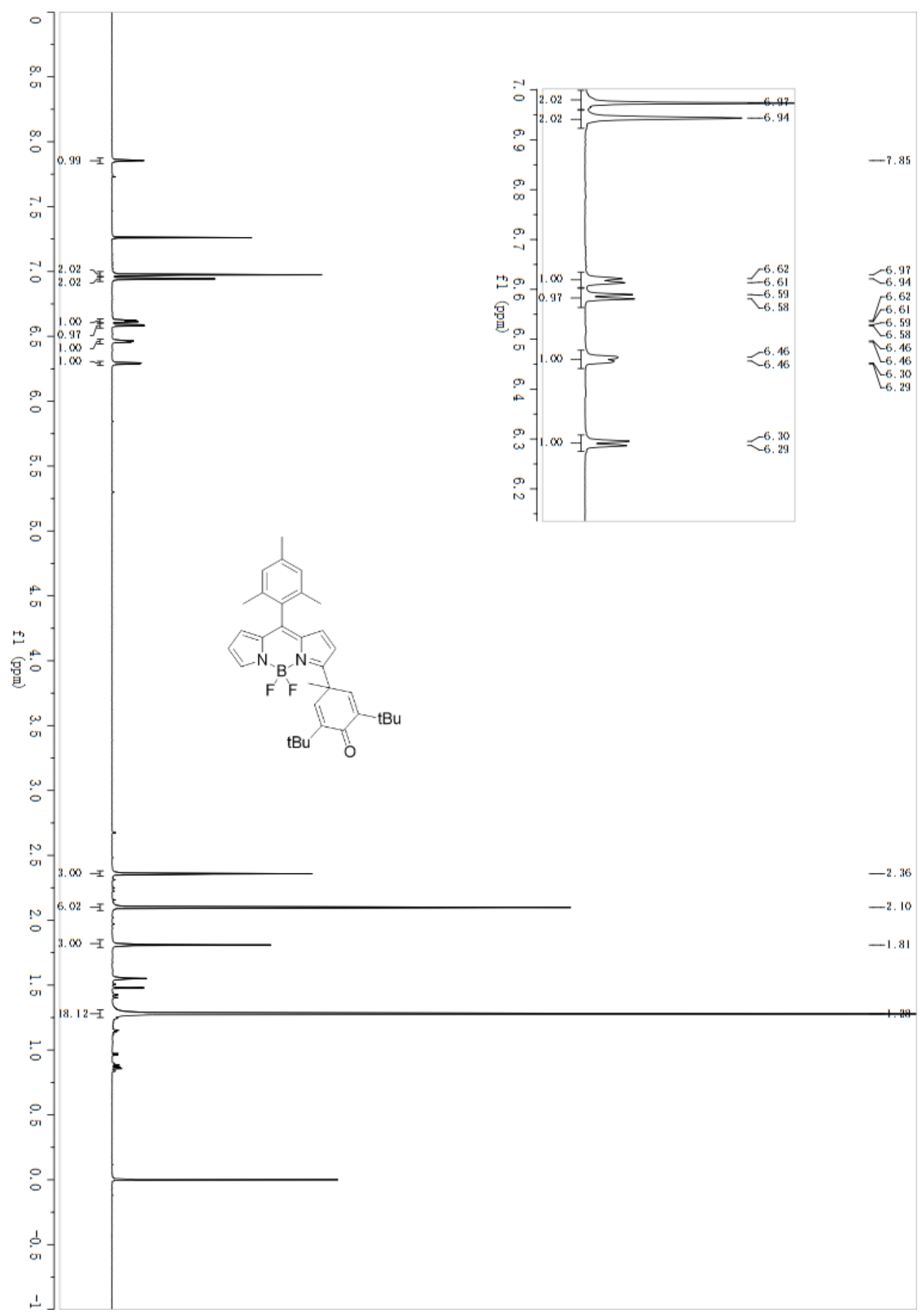

${ }^{1} \mathrm{H}$ NMR spectrum of $9 \mathbf{a}\left(\mathrm{CDCl}_{3}, 500 \mathrm{MHz}\right)$ 


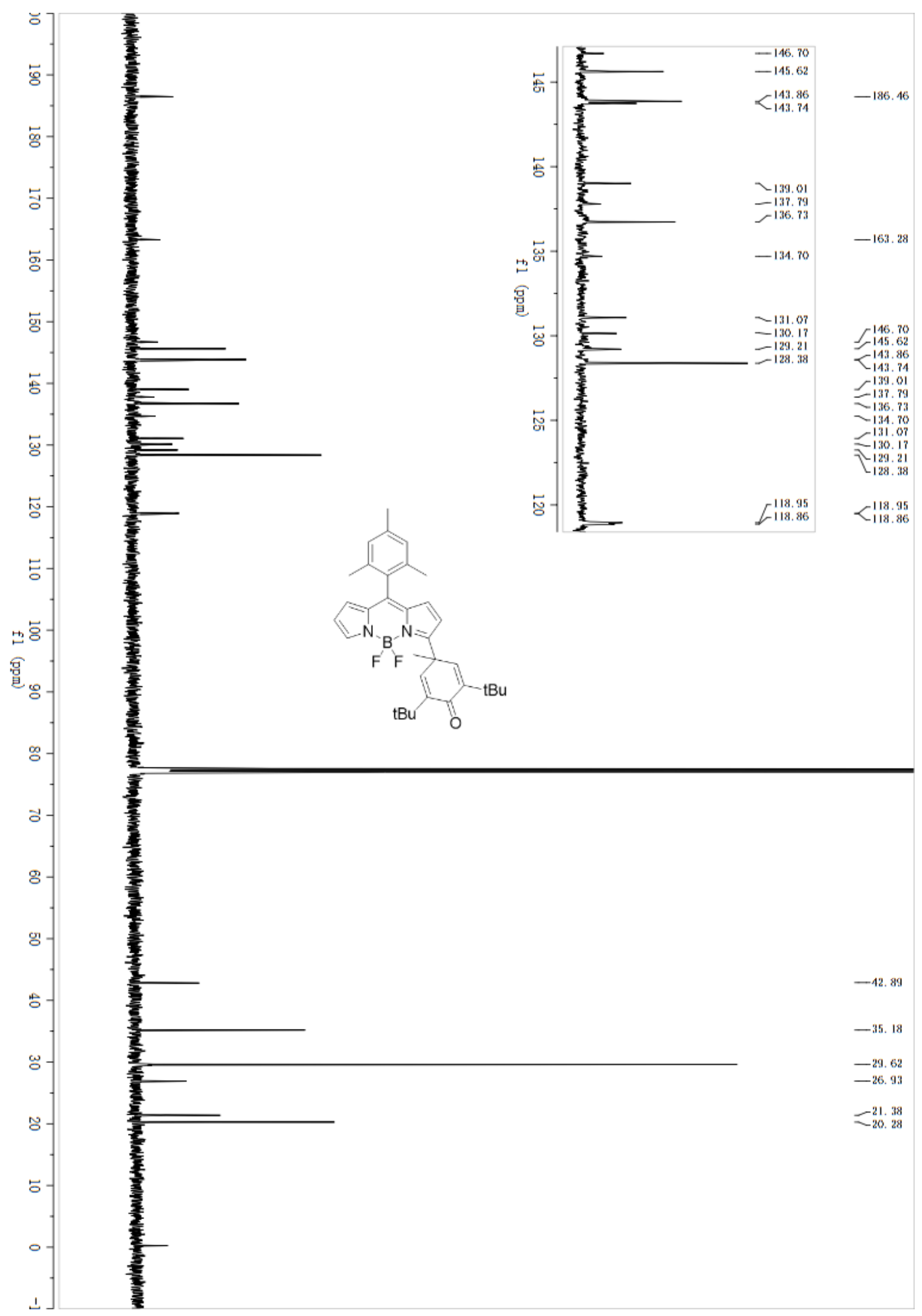

${ }^{13} \mathrm{C}$ NMR spectrum of $\mathbf{9 a}\left(\mathrm{CDCl}_{3}, 126 \mathrm{MHz}\right)$ 


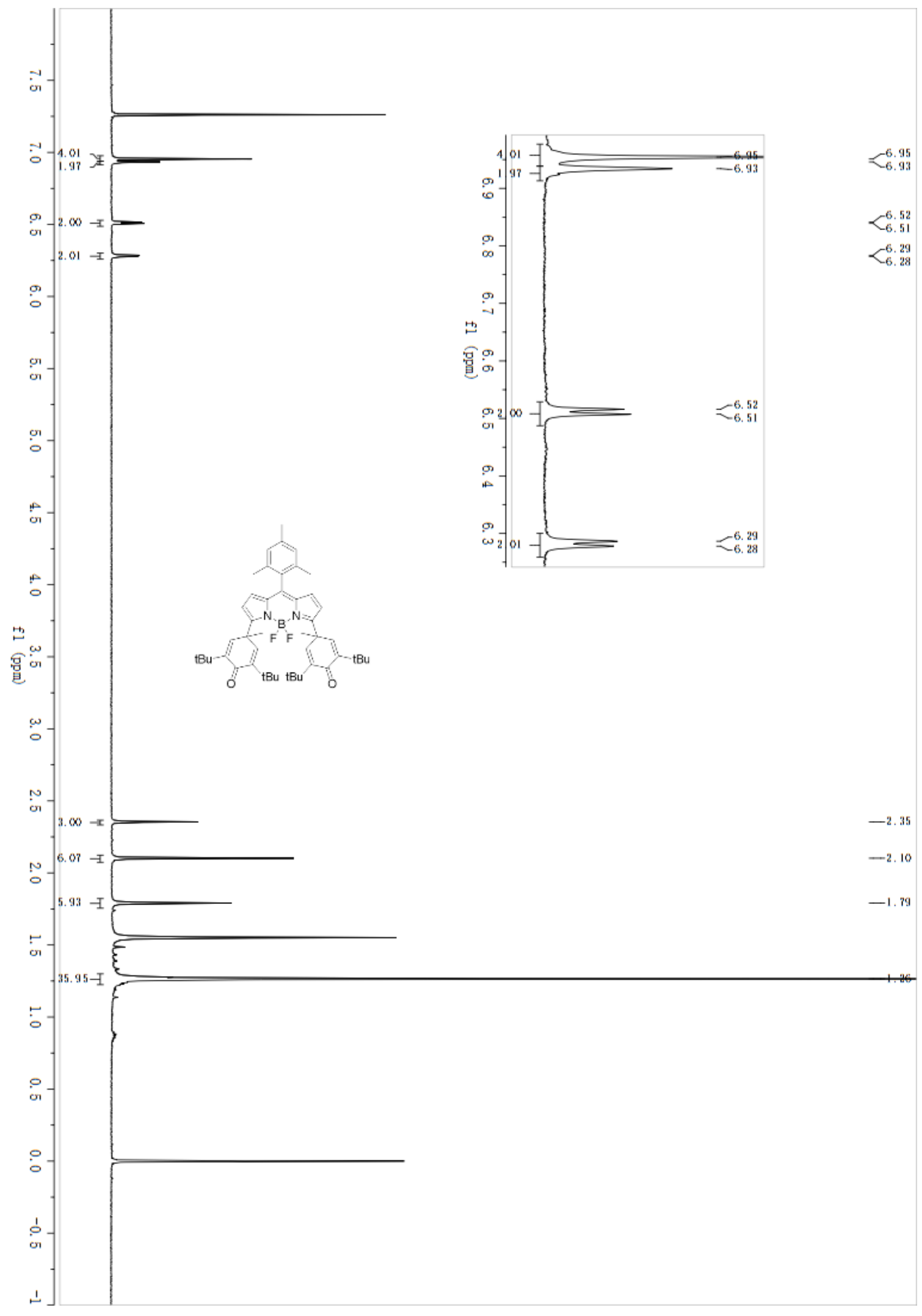

${ }^{1} \mathrm{H} \mathrm{NMR}$ spectrum of $\mathbf{9 b}\left(\mathrm{CDCl}_{3}, 500 \mathrm{MHz}\right)$ 


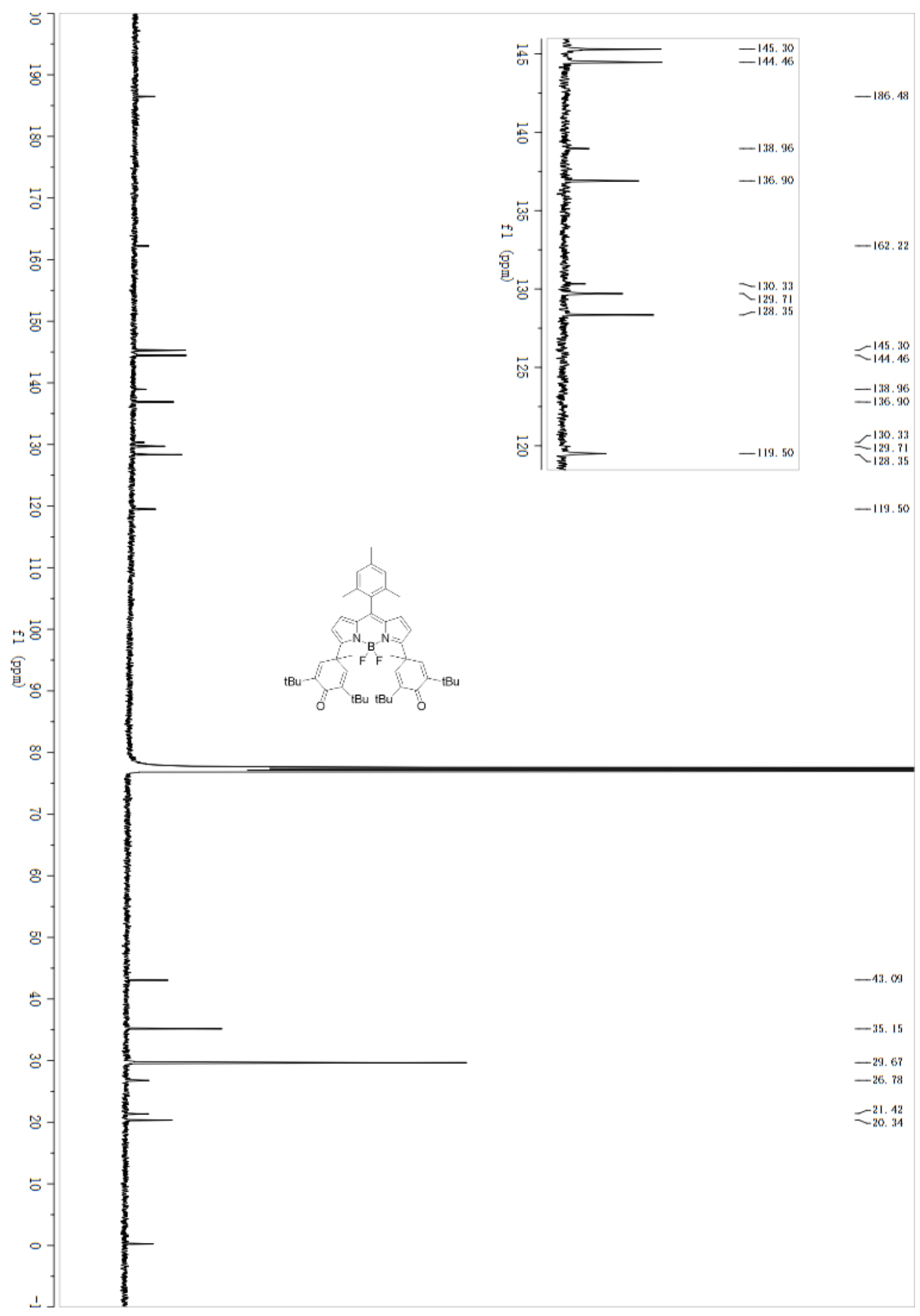

${ }^{13} \mathrm{C}$ NMR spectrum of $\mathbf{9 b}\left(\mathrm{CDCl}_{3}, 126 \mathrm{MHz}\right)$ 


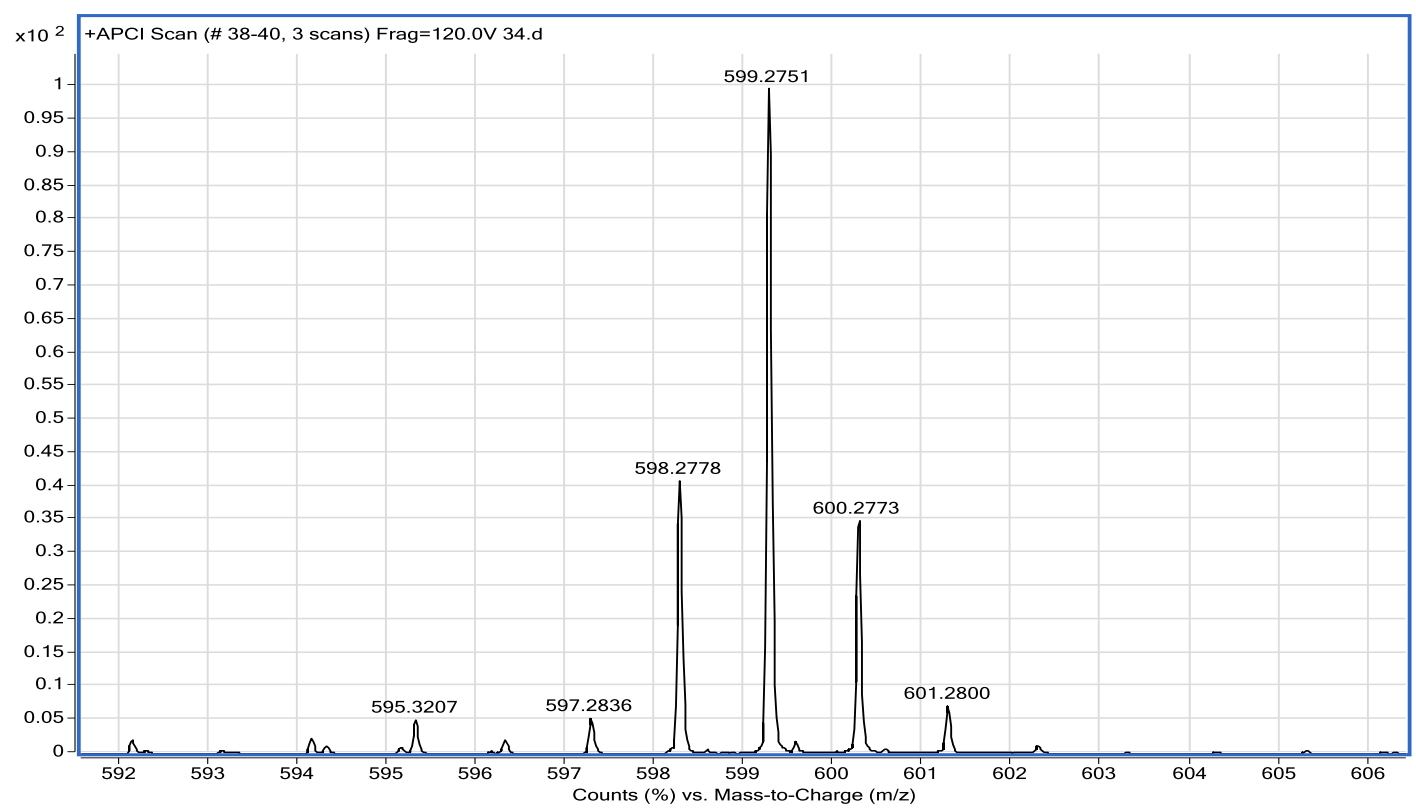

HRMS for $\mathbf{2 a}$

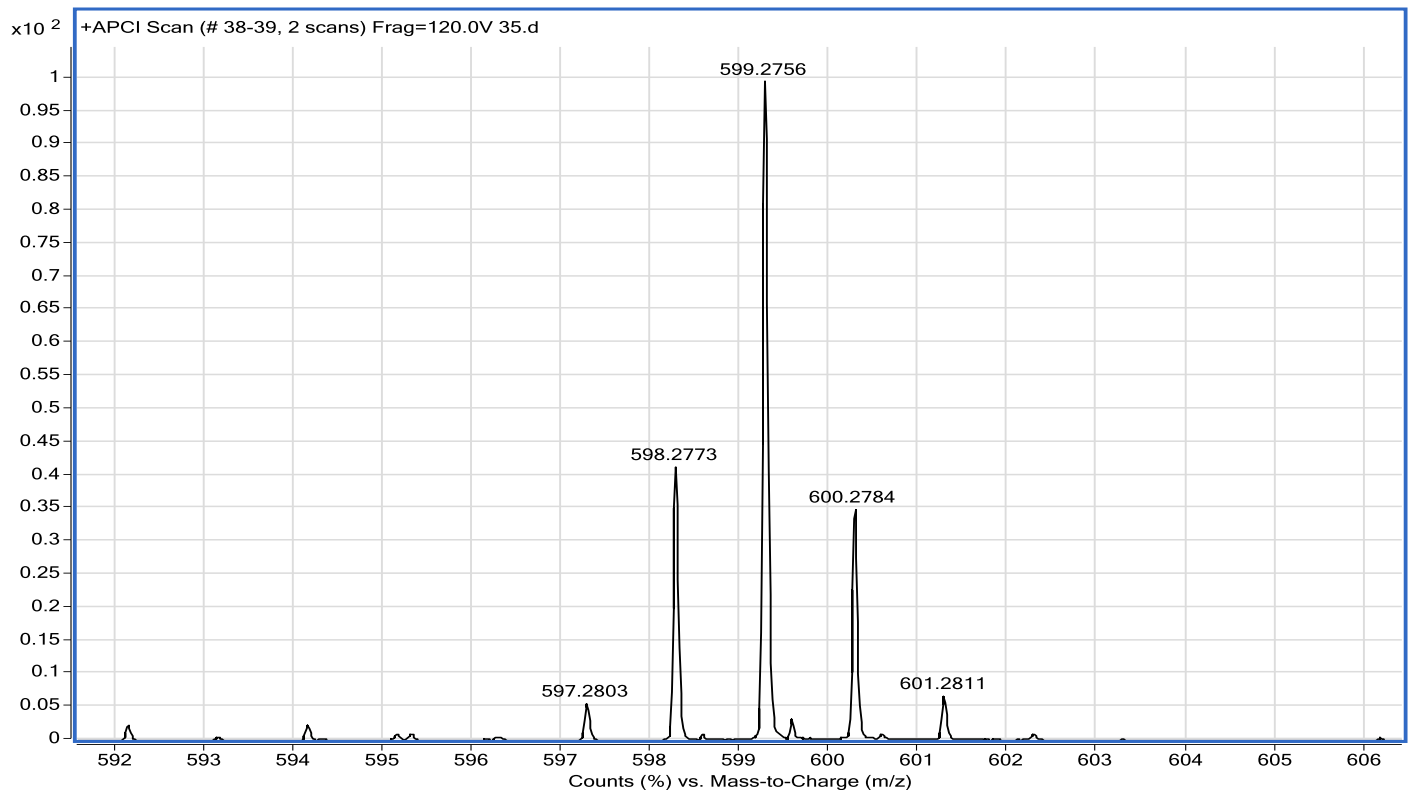

HRMS for 3a 


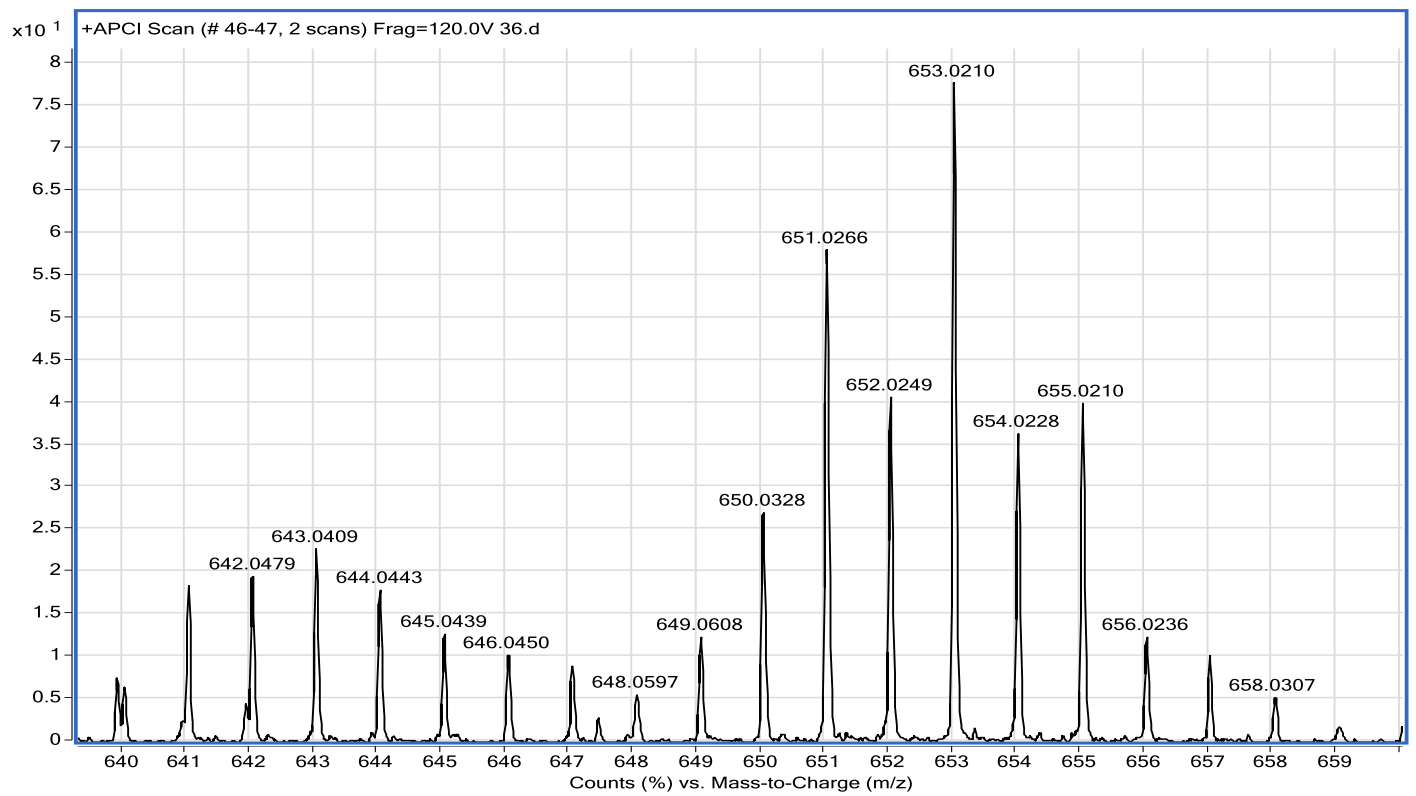

HRMS for $\mathbf{2 b}$

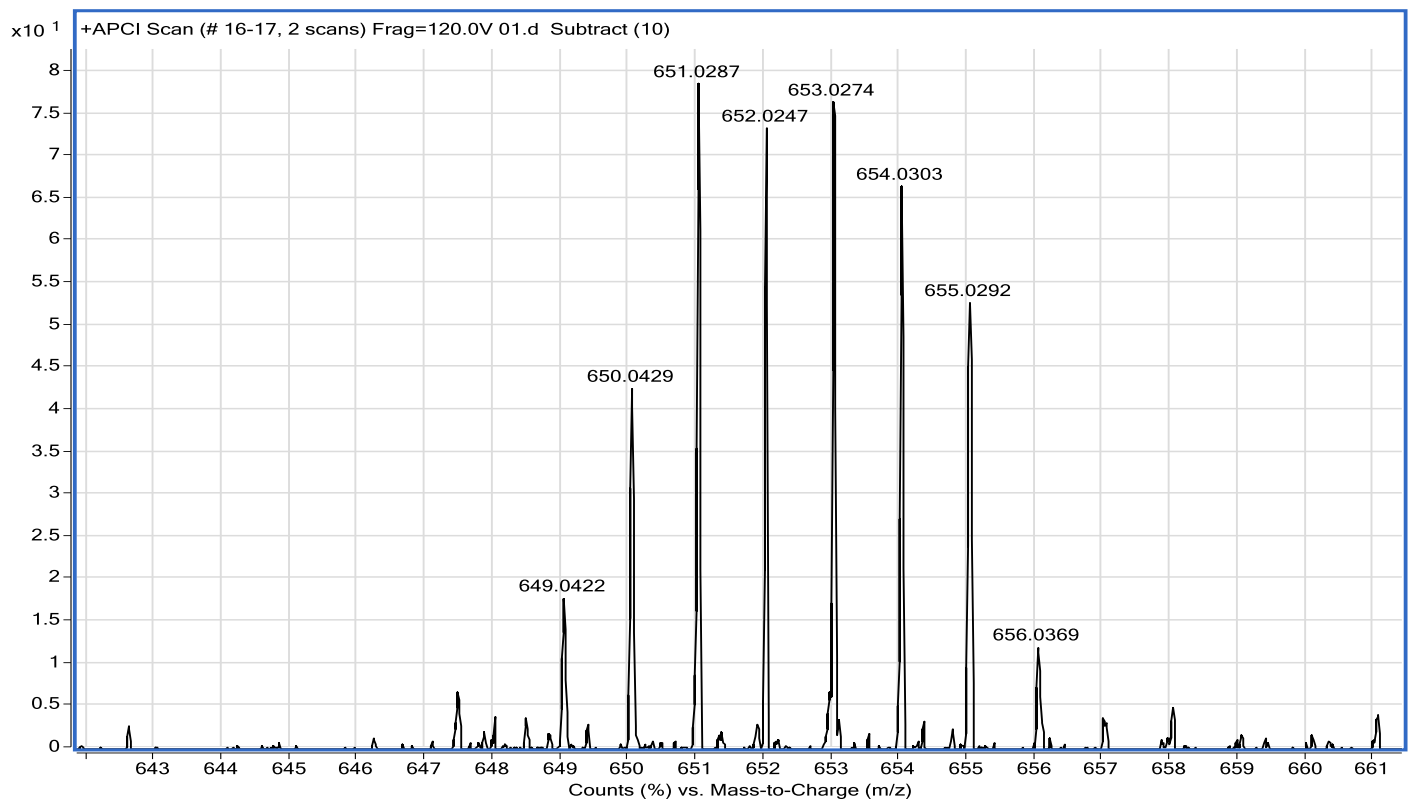

HRMS for $\mathbf{3 b}$ 


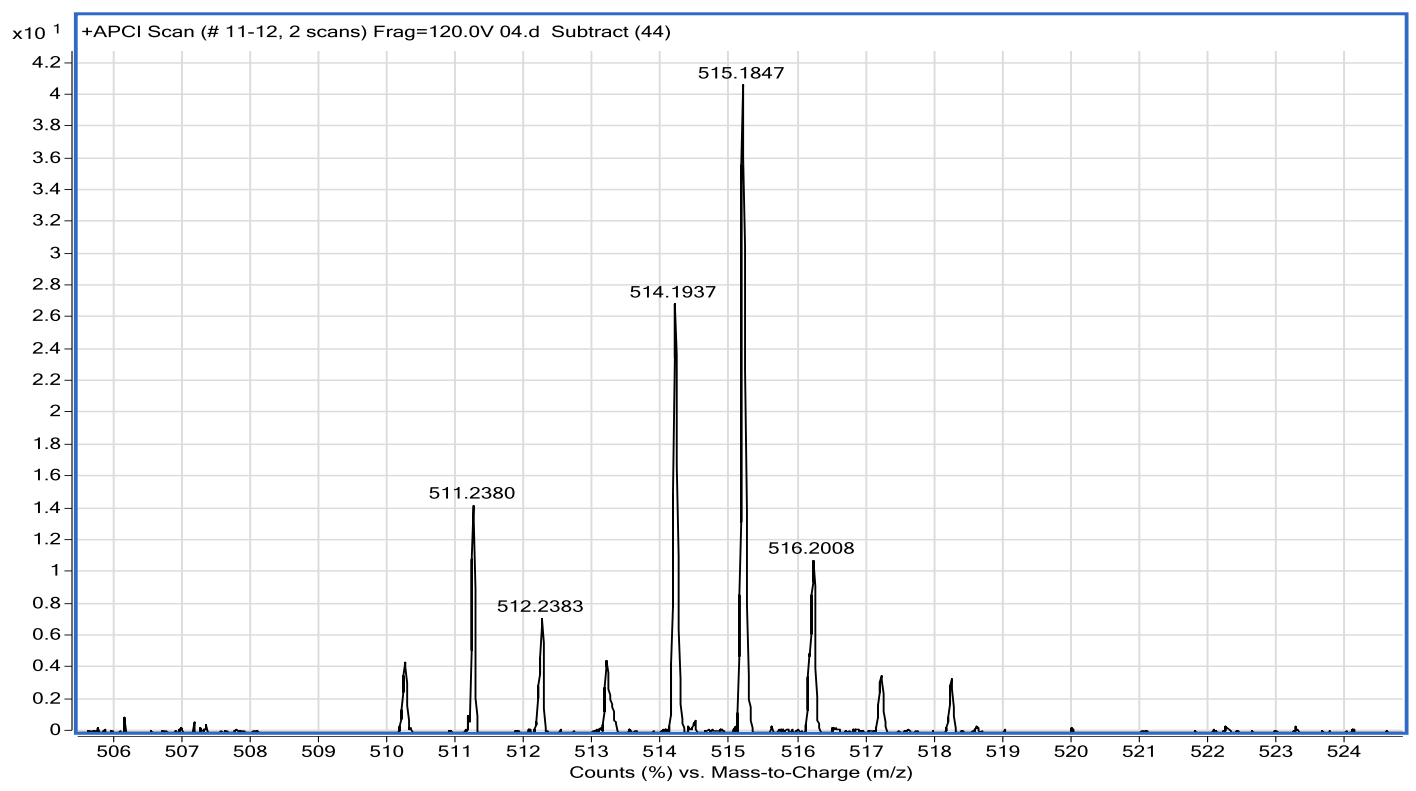

HRMS for 2c

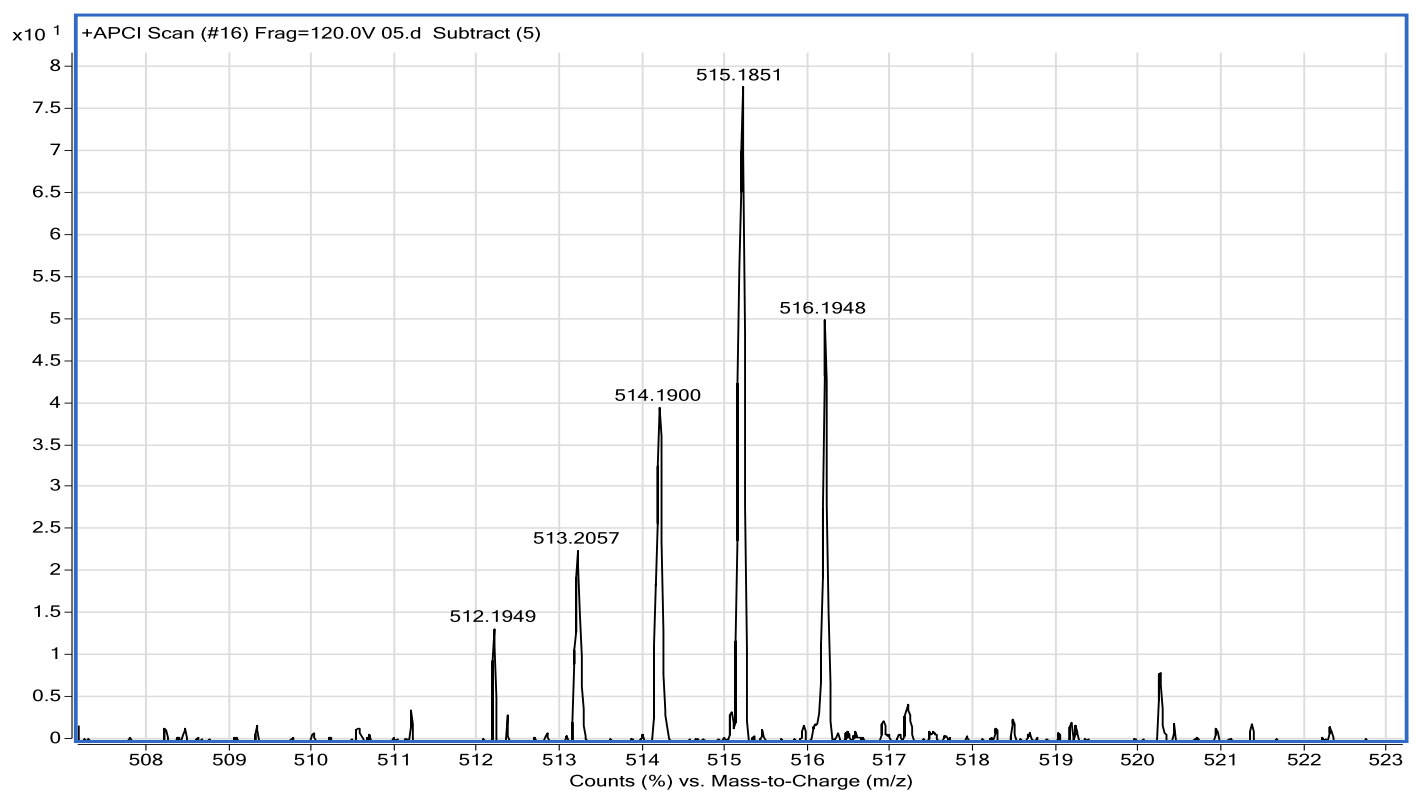

HRMS for $\mathbf{3 c}$ 


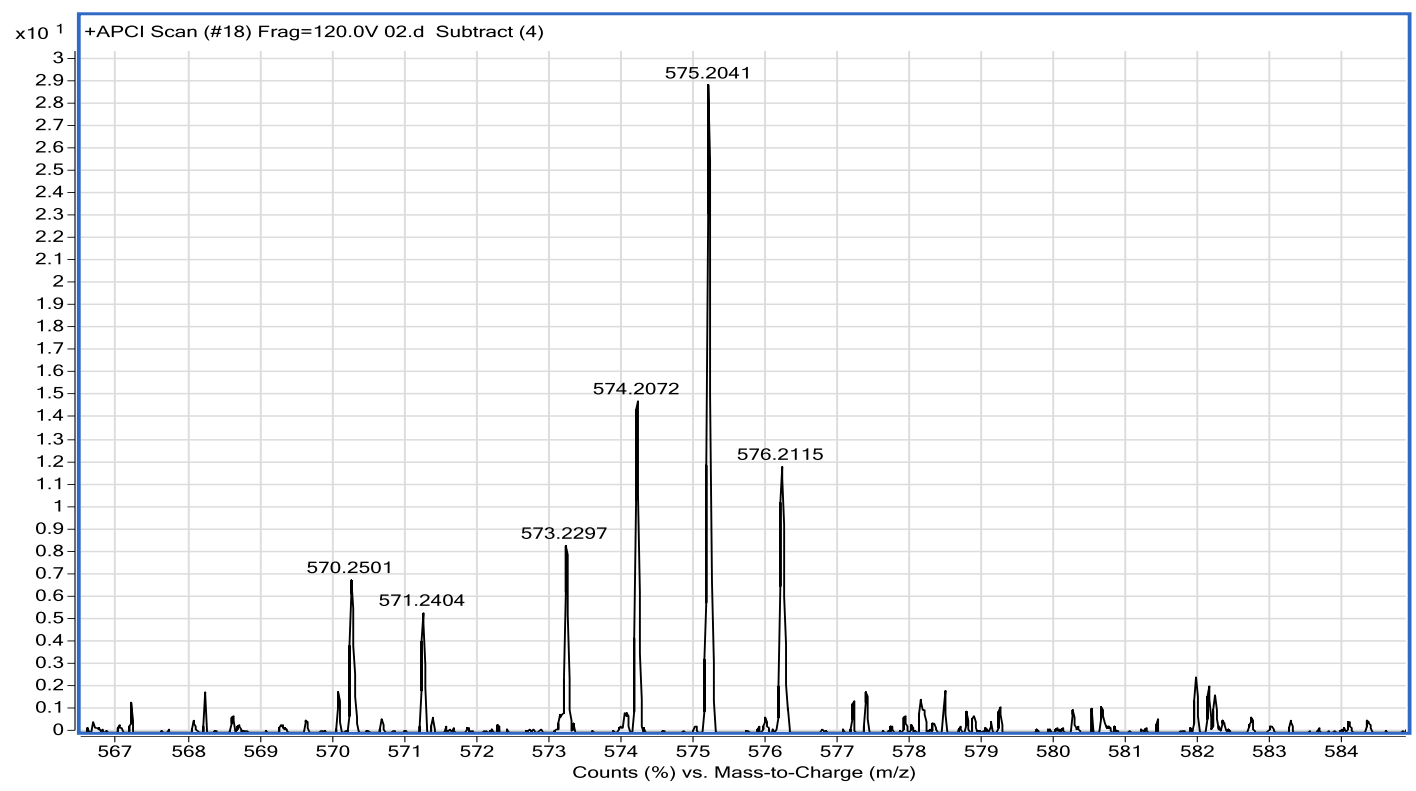

HRMS for 2d

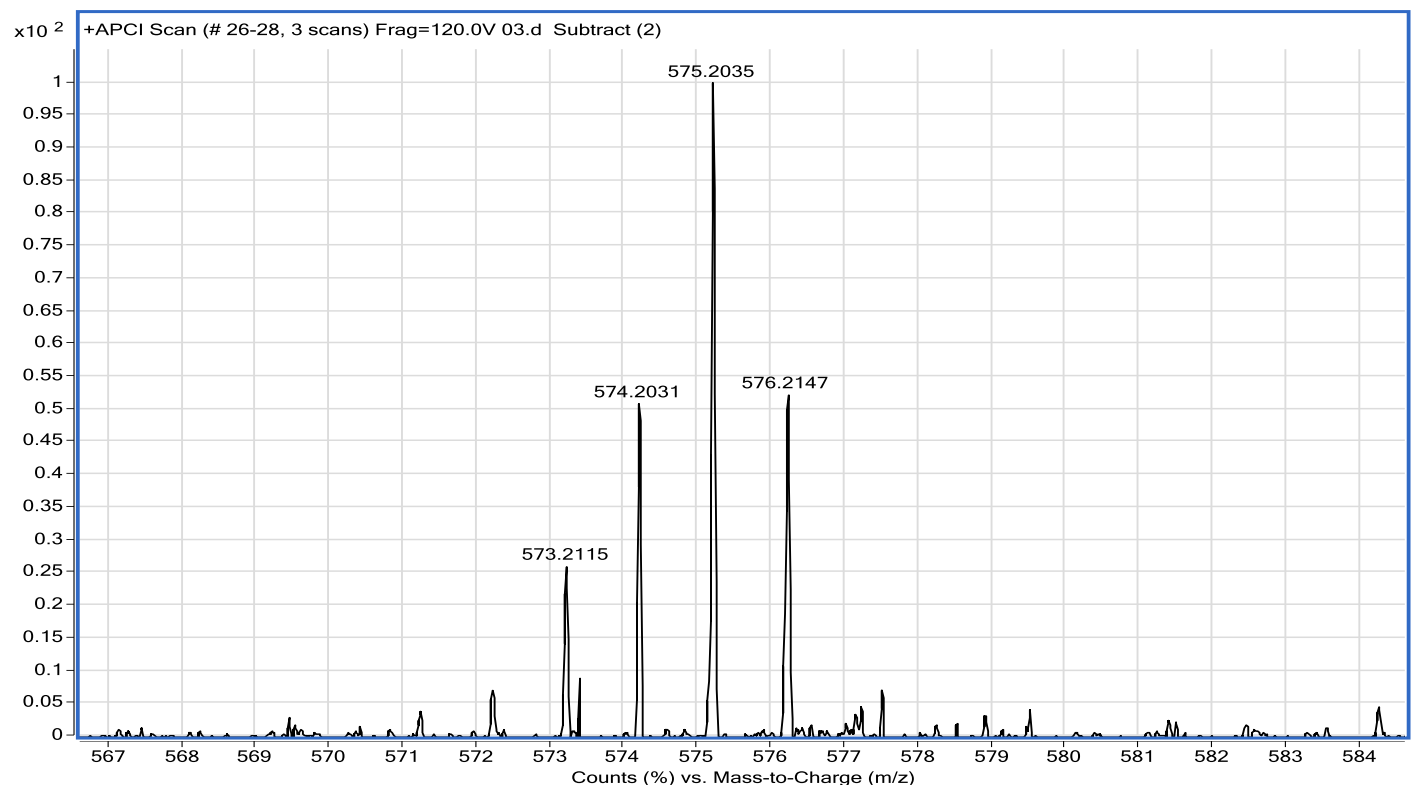

HRMS for 3d 


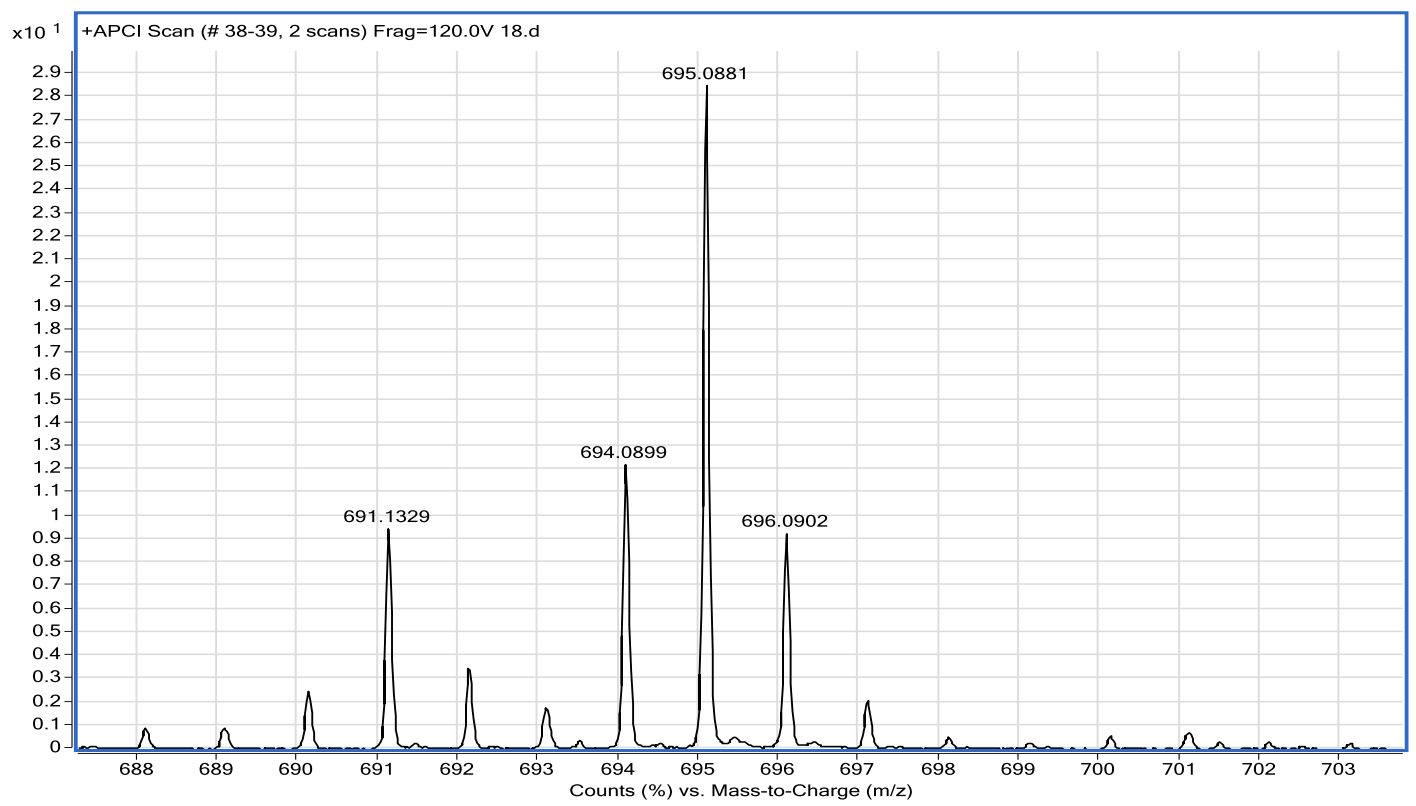

HRMS for $\mathbf{2 e}$

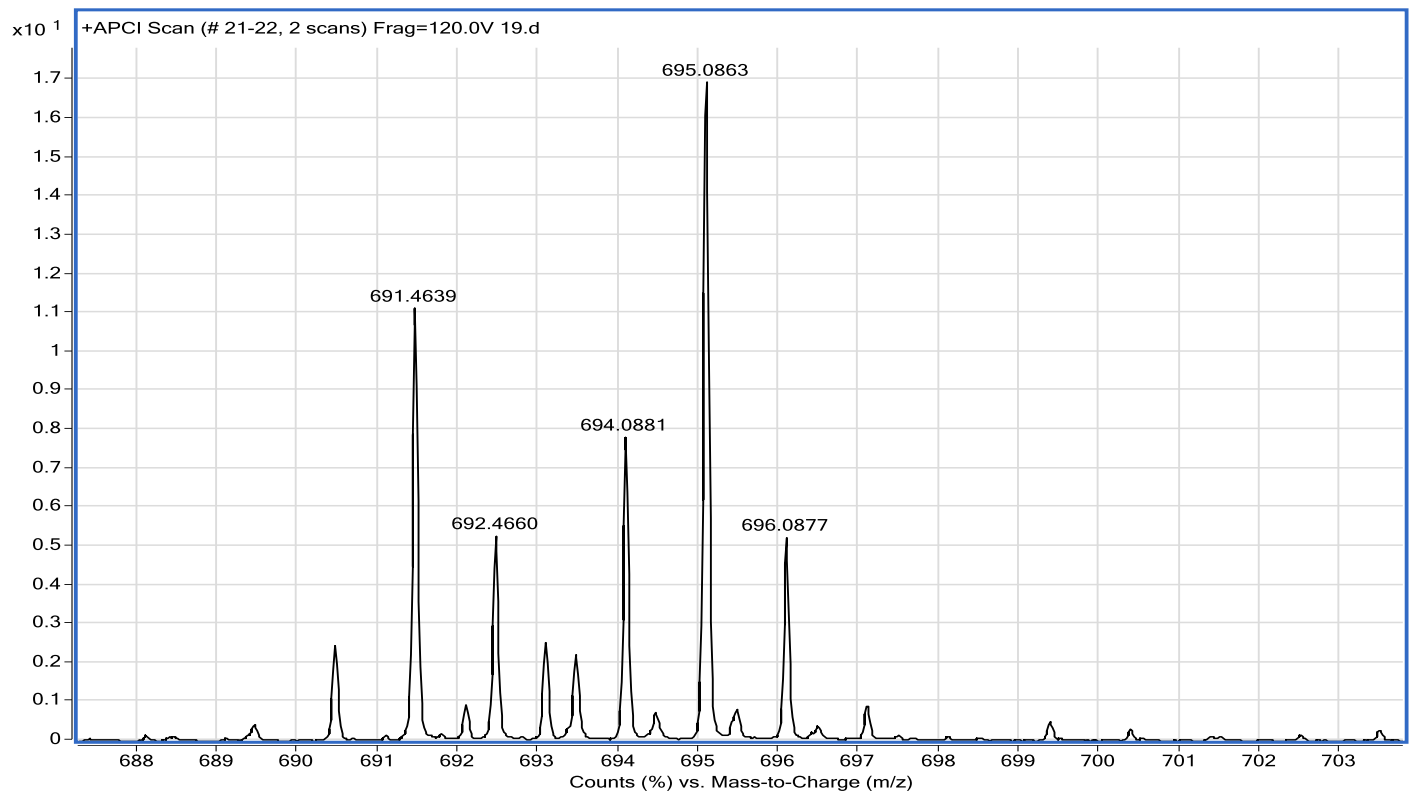

HRMS for 3e 

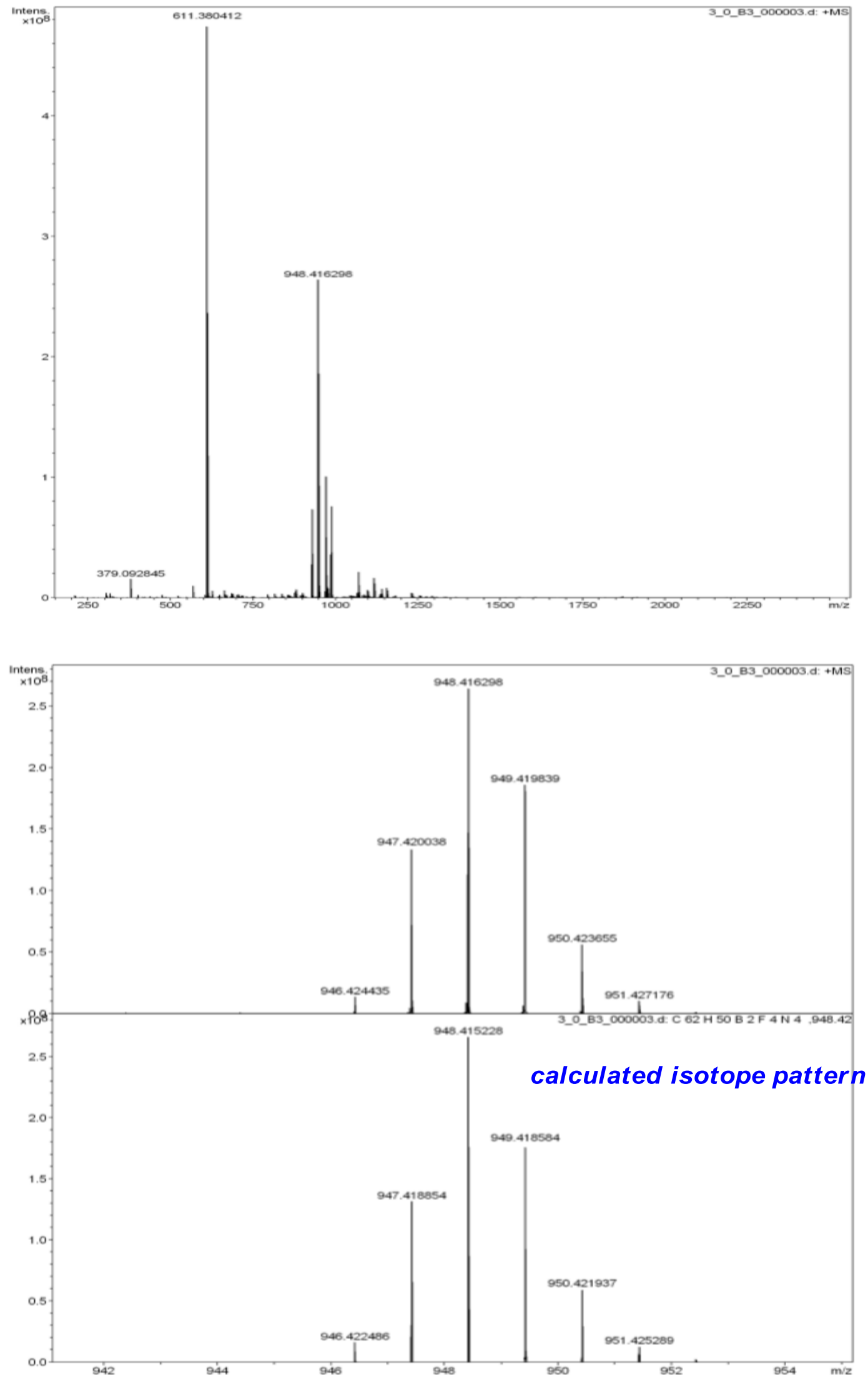

HRMS for 5 

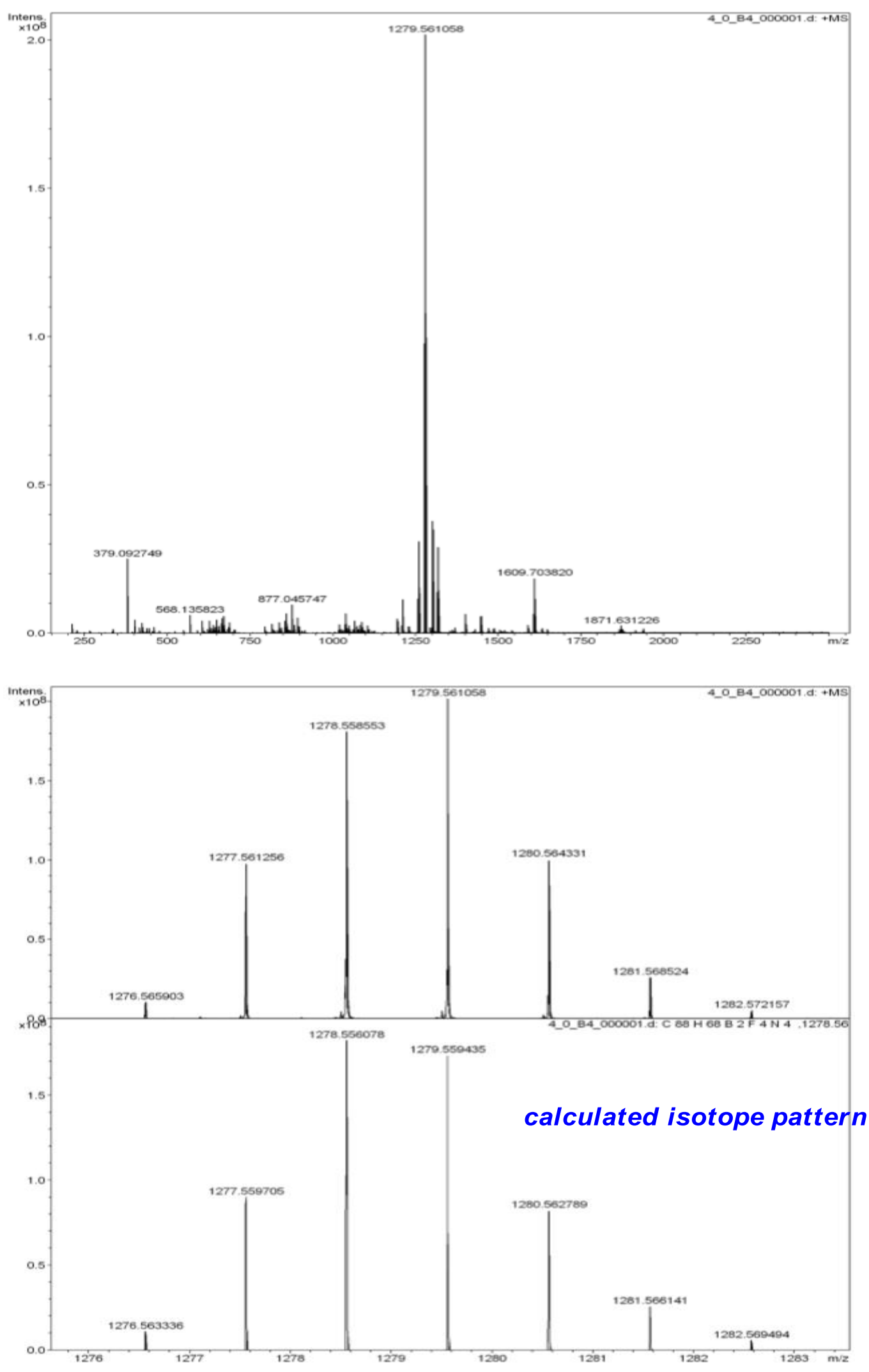

HRMS for 6

S84 / S88 


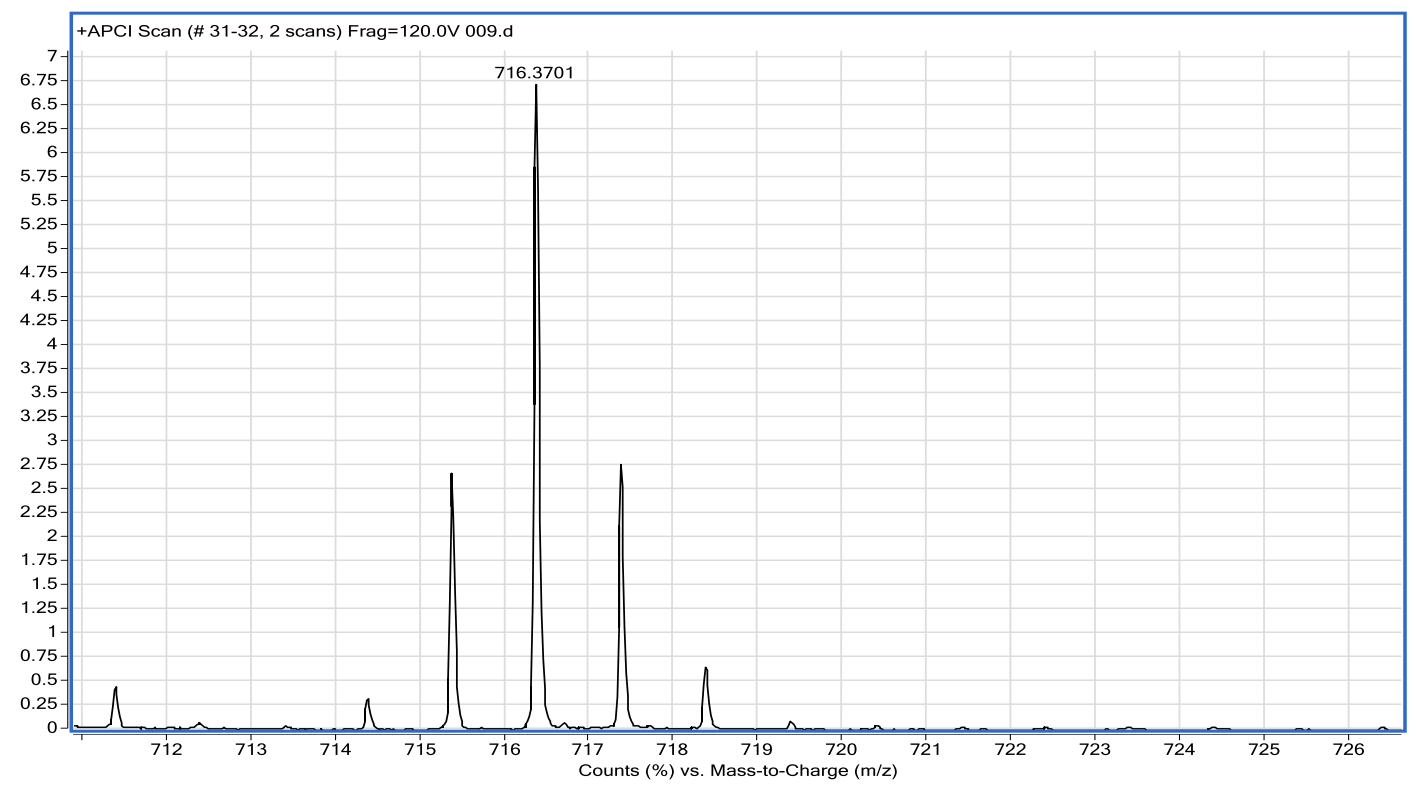

HRMS for 7

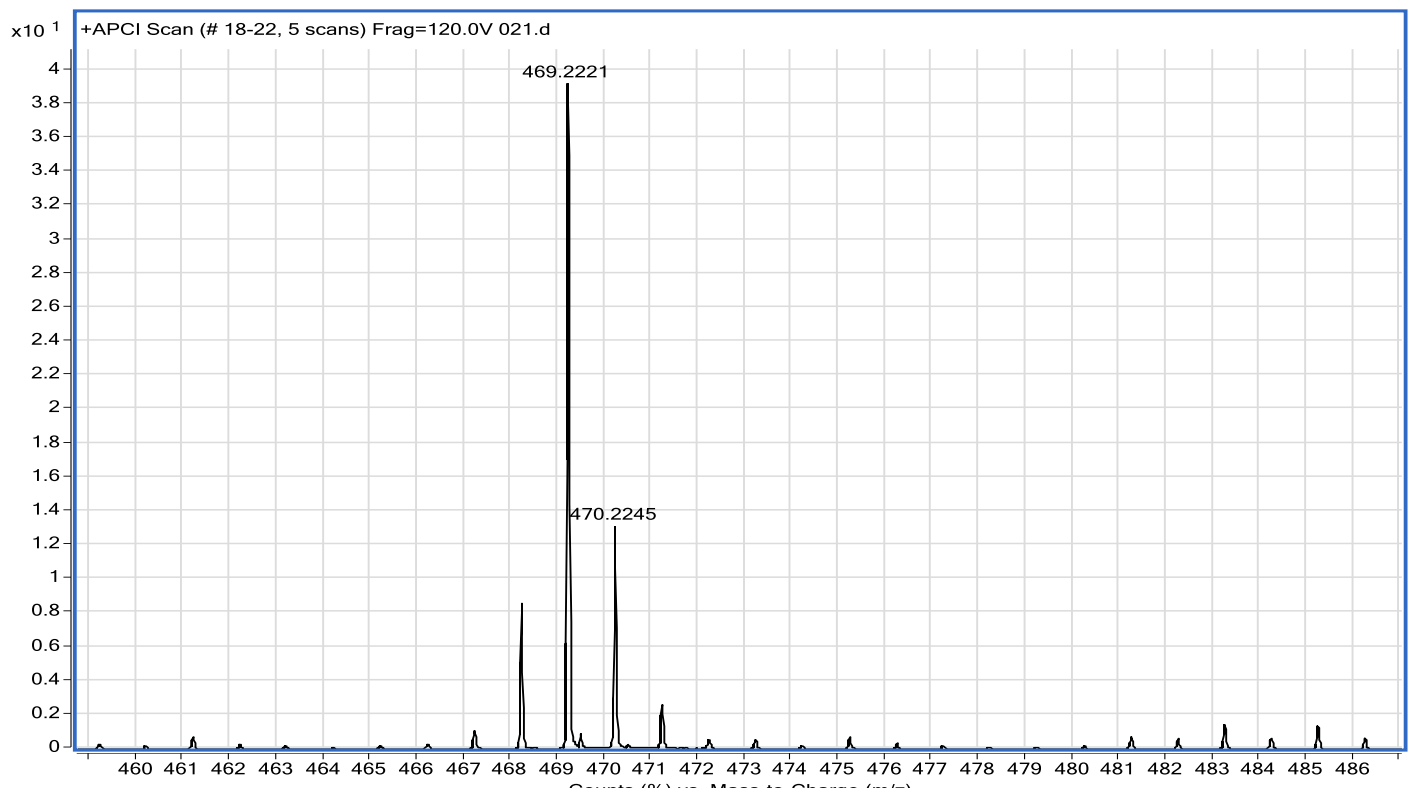

HRMS for 8 


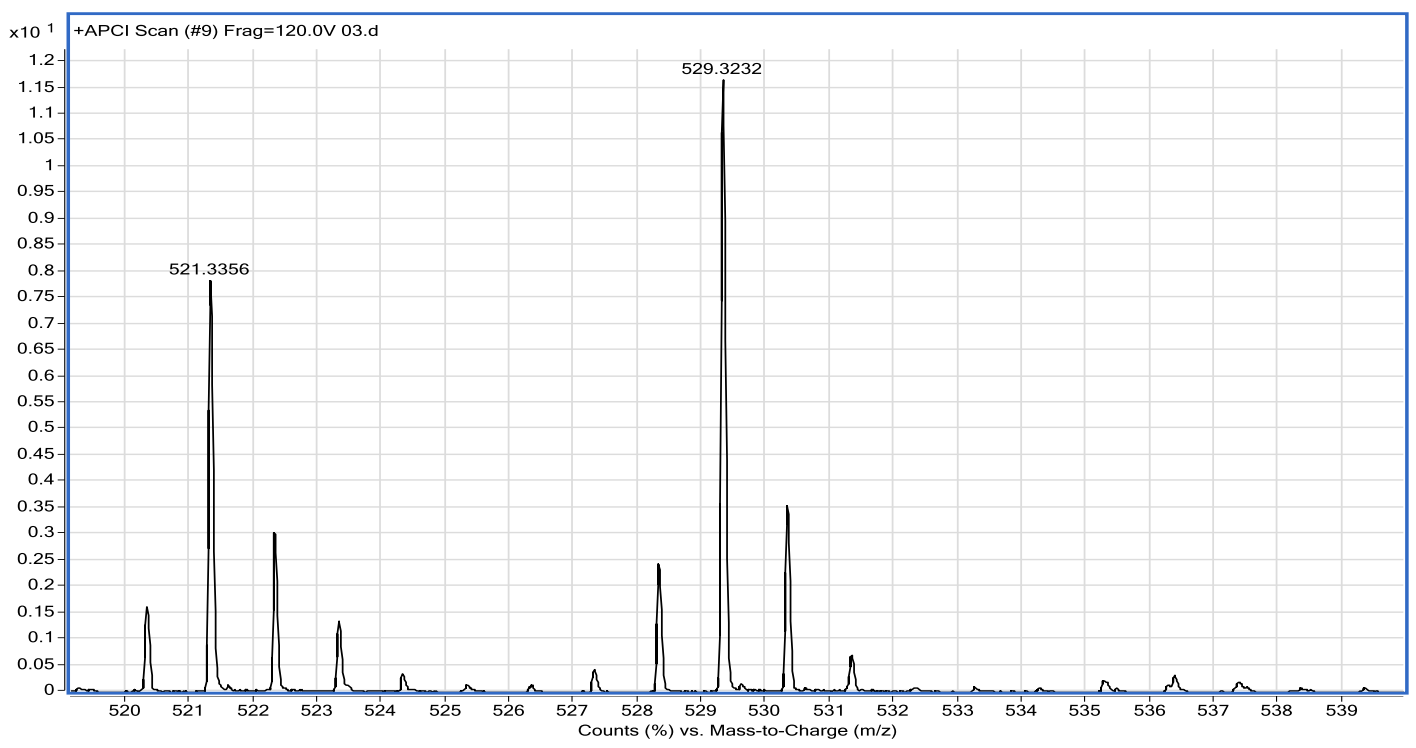

\section{HRMS for 9a}

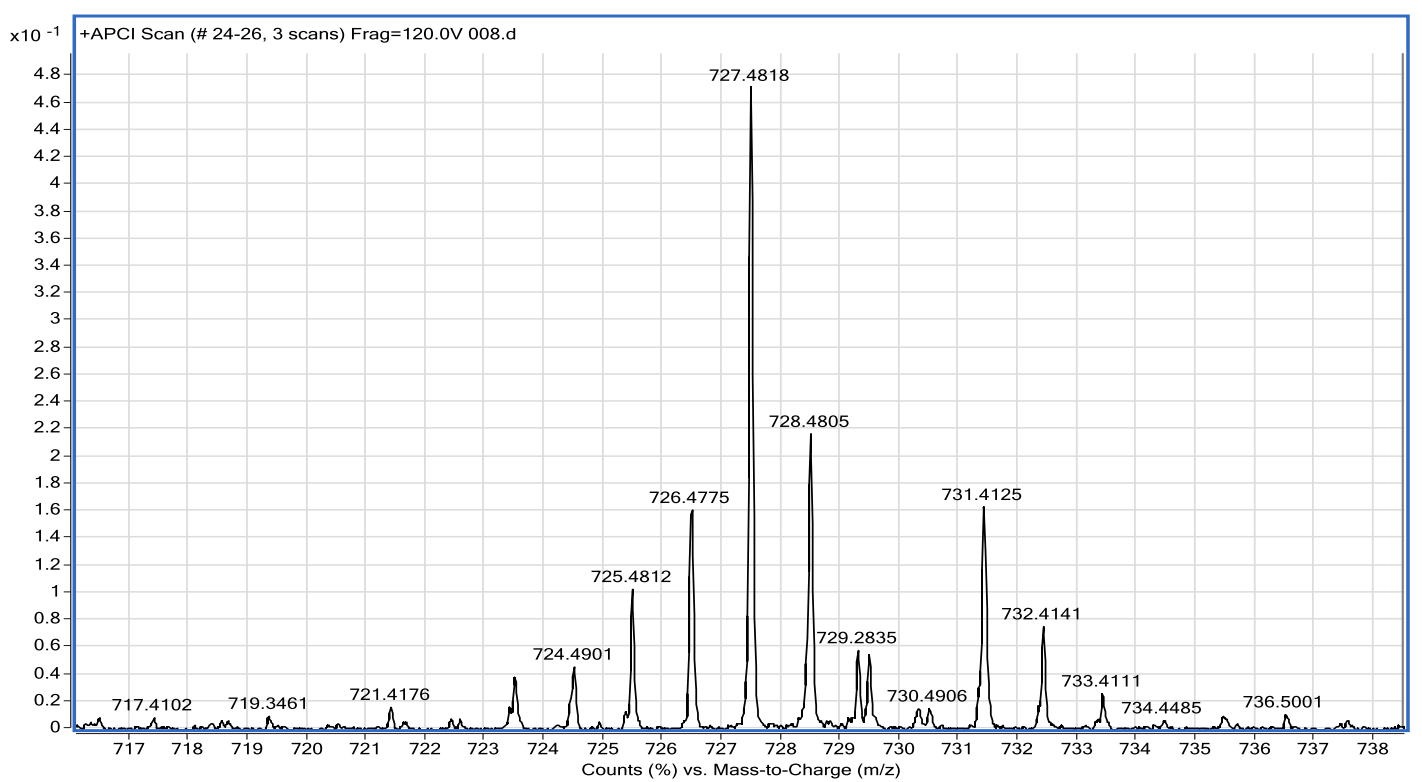

HRMS for $\mathbf{9 b}$ 


\section{REFERENCES}

1) Isak, S.; Eyring, E. J. Phys. Chem. 1992, 96, 1738.

2) Vieira F. L.; Cabral, P.; Almeida, P.; Oliveira, A.; Reis, M.; Botelho do Rego, A. .Macromolecules 1998, 31, 3936-3944.

3) Olmsted, J. J. Phys. Chem. 1979, 83, 2581.

4) (a) Killoran, J.; Allen, L.; Gallagher, J. F.; Gallagher, W. M.; O’Shea. D. F. Chem. Commun. 2002, 1862. (b) McDonnell, S. O.; Hall, M. J.; Allen, L. T.; Byrne, A.; Gallagher, W. M.; O'Shea, D. F. J. Am. Chem. Soc. 2005, 127, 16360.

5) Benson, R. C.; Kues, H. A. Phys. Med. Biol., 1978, 23, 159

6) Software for the integration of CCD detector system Bruker analytical X-ray systems, Bruker axs, Madison, WI (after 2013).

7) APEX2 suite for crystallographic software, Bruker axs, Madison, WI.

8) Dolomanov, O.; BourhisL, J.; Gildea, R.; Howard, J.; Puschmann, H. Olex2: A complete structure solution, refinement and analysis program, J. Appl. Cryst, 2009, $42,339$.

9) Sheldrick, G.M. Crystal structure refinement with ShelXL, Acta Cryst, 2015, A71, 3 .

10) a) Treat, N.; Sprafke, H.; Kramer, J.; Clark, P.; Barton, B.; Fors, B.; Hawker, C. J. Am. Chem. Soc. 2014, 136, 16096. b) Tucker, J.; Stephenson, C. J. Org. Chem . 2012, 77, 1617.

11) Jasinska, K.; Shan, W.; Zawada, K.; Kadish,K.; Gryko, D. J. Am. Chem. Soc. 2016, $138,15451$.

12) Silvi, M.; Verrier, C.; Rey, Y.; Buzzetti, Luca.; Melchiorre, P. Nat. Chem. 2017, 9, 868.

13) Yu, C.; Jiao, L.; Yin, H.; Zhou, J.; Pang, W.; Wu, Y.; Wang, Z.; Yang, G.; Hao, E. Eur. J. Org. Chem. 2011, 2011, 5460.

14) Ventura, B.; Marconi, G.; Bröring, M.; Krüger, R.; Flamigni, L. New J. Chem. 2009, 33, 428.

15) Cakmak, Y.; Kolemen, S.; Duman, S.; Dede,Y.; Dolen, Y.; Kilic, B.; Kostereli, Z.; Yildirim, L. T.; Dogan, A. L.; Guc, D.; Akkaya, E. U. Angew. Chem., Int. Ed. 2011, 50, 11937.

16) Pang, W.; Zhang, X.; Zhou, J.; Yu, C.; Hao, E.; Jiao, L. Chem. Commun. 2012, 48, 5437.

17) Rihn, S.; Erdem, M.; Nicola, A. D.; Retailleau, P.; Ziessel, R. Org. Lett. 2011, 13, 1916; 
18) Hayashi, Y.; Yamaguchi, S.; Cha, W. Y.; Kim, D.; Shinokubo, H. Org. Lett. 2011, 13, 2992.

19) Yu, C.; Jiao, L.; Li ,T.; Wu, Q.; Miao, W.; Wang, J.; Wei, Y.; Hao, E. Chem. Commun. 2015, 51, 16852.

20) a) Nakamura, M.; Tahara, H.; Takahashi, K.; Nagata, T.; Uoyama, H.; Kuzuhara, D.; Mori, S.; Okujima, T.; Yamada, H.; Uno, H. Org. Biomol. Chem. 2012, 10, 6840; b) Nakamura, M.; Kitatsuka, M.; Takahashi, K.; Nagata, T.; Mori, S.; Kuzuhara, D.; Okujima, T.; Yamada, H.; Nakae, T.; Uno, H. Org. Biomol. Chem. 2014, 12, 1309.

21) Wakamiya, A.; Murakami, T.; Yamaguchi, S. Chem. Sci. 2013, 4, 1002.

22) Ni, Y.; Lee, S.; Son, M.; Aratani, N.; Ishida, M.; Samanta, A.; Yamada, H.; Chang, Y.; Furuta, H.; Kim, D.; Wu, J. Angew. Chem., Int. Ed. 2016, 55, 2815.

23) a) Usui, Y. Chem. Lett. 1973, 2, 743. b) Li, W.; Li, L.; Xiao, H.; Qi, R.; Huang, Y.; Xie, Z.; Jing, X.; Zhang, H. RSC Adv. 2013, 3, 13417; c) Feng, Z.; Feng, Y.; Yu, C.; Dai, E.; Wei, Y.; Mu, X.; Jiao, L.; Hao, E. J. Org. Chem. 2016, 81, 6281. 